\title{
Design, Synthesis, and Evaluation of
}

\section{Fluorinated Radioligands for Myelin Imaging}

Anand Dev Tiwari, ${ }^{\dagger \dagger}$ Chunying Wu, ${ }^{\dagger \dagger, ~}$ Junqing Zhu, ${ }^{\dagger}$ Sheng Zhang, ${ }^{\dagger}$ Jinle Zhu, ${ }^{\dagger, \circ}$

William R. Wang, ${ }^{\dagger, \nabla}$ Jinming Zhang, ${ }^{\ddagger}$ Curtis Tatsuoka, ${ }^{\S}$ Paul M. Matthews, ${ }^{\|}$Robert H. Miller, ${ }^{\perp}$ and Yanming Wang ${ }^{*} \dagger$

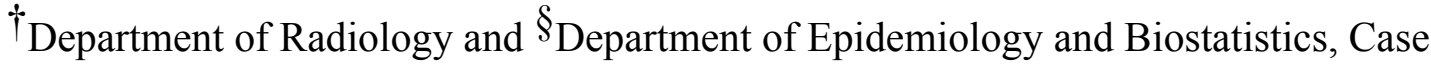
Western Reserve University, Cleveland, Ohio 44106, United States

\$Department of Nuclear Medicine, People’s Liberation Army (PLA) General Hospital, Beijing 100853, China

"Division of Brain Sciences, Department of Medicine, Imperial College London SW12 0NN, United Kingdom

${ }^{\perp}$ Department of Anatomy and Regenerative Biology, George Washington University, Washington, DC 20037, United States

*Corresponding Author: E-mail: yxw91@case.edu. Phone: 216-844-3288. Fax: 216-8448062.

Present Addresses: ${ }^{\circ}$ Beachwood High School, Beachwood, OH 44122, United 1043 States.

${ }^{\nabla}$ Phillips Academy, Andover, MA 01810, United States. 


\section{Contents:}

\section{Spectra:}

1. ${ }^{1} \mathrm{H}^{13} \mathrm{C}$ and ${ }^{19} \mathrm{~F}$ NMR Spectra for all the Intermediate and final compounds Synthesized.

2. HRMS Spectra for the all the final compounds $(\mathbf{2 5}, \mathbf{3 2 - 3 7}, \mathbf{3 9})$.

3. HPLC Spectra for the all the final compounds $(\mathbf{2 5}, \mathbf{3 2 - 3 7}, \mathbf{3 9})$.

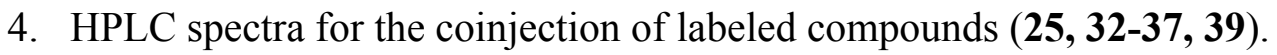

\section{Table:}

Table S1. Ex vivo staining of all compounds $(\mathbf{2 5}, \mathbf{3 2 - 3 7 , 3 9 )}$

Movie Clip:

MircoPET/CT 3D movie of the whole brain and part of spinal cord imaging for the compound $\mathbf{3 2}$ 

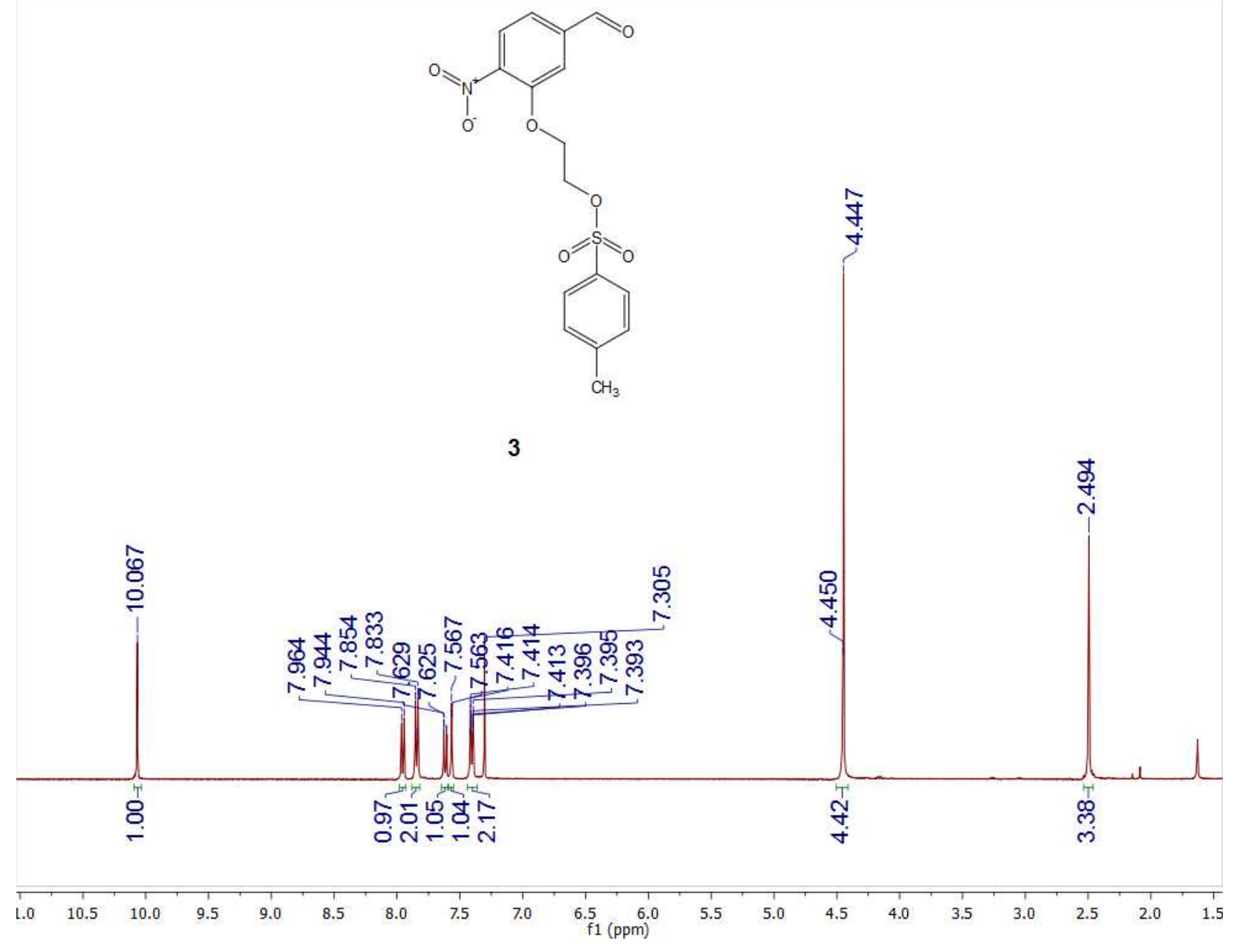
足

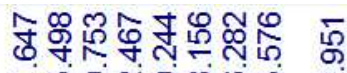

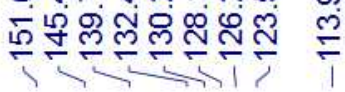

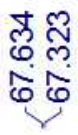

$\frac{\bar{\sigma}}{\text { ஸे }}$

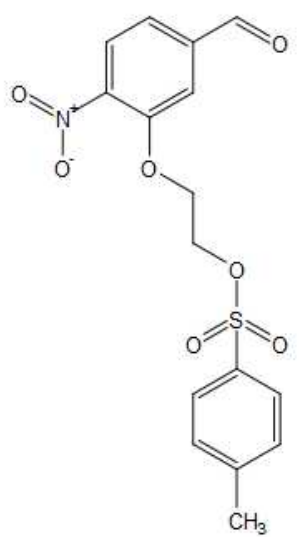

3
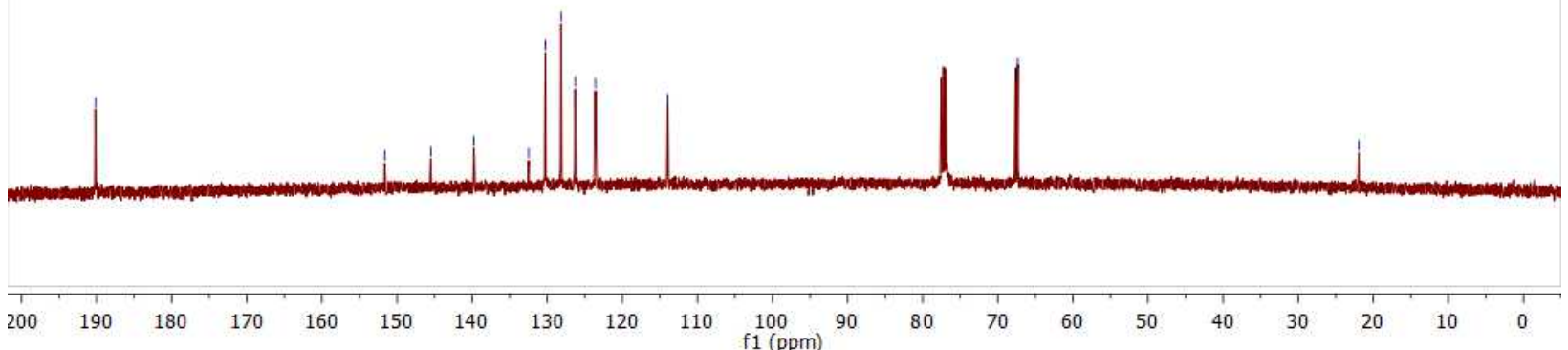

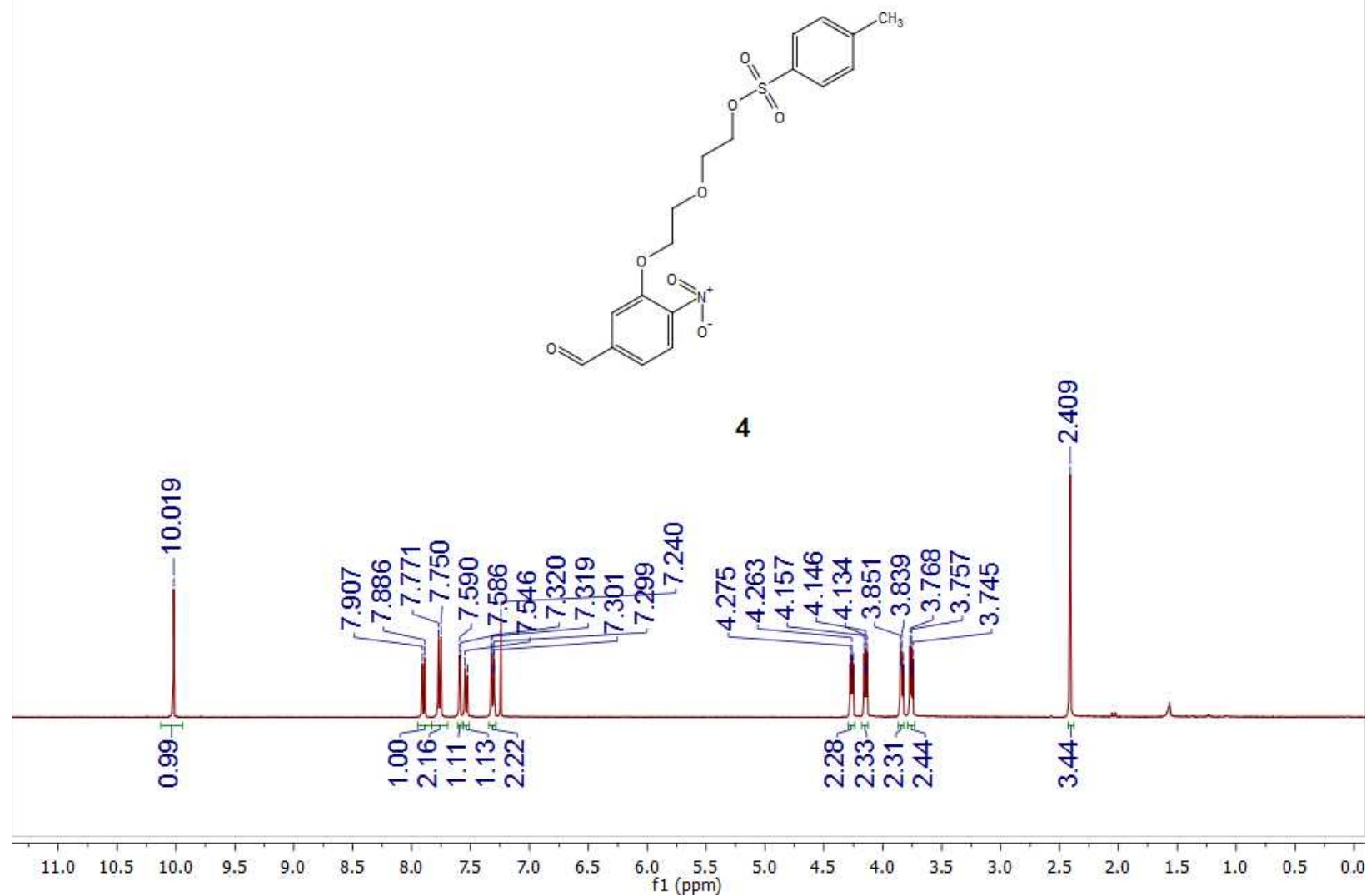
앙

๓๐్

กิ่

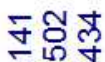

용

4

ষ্ণ
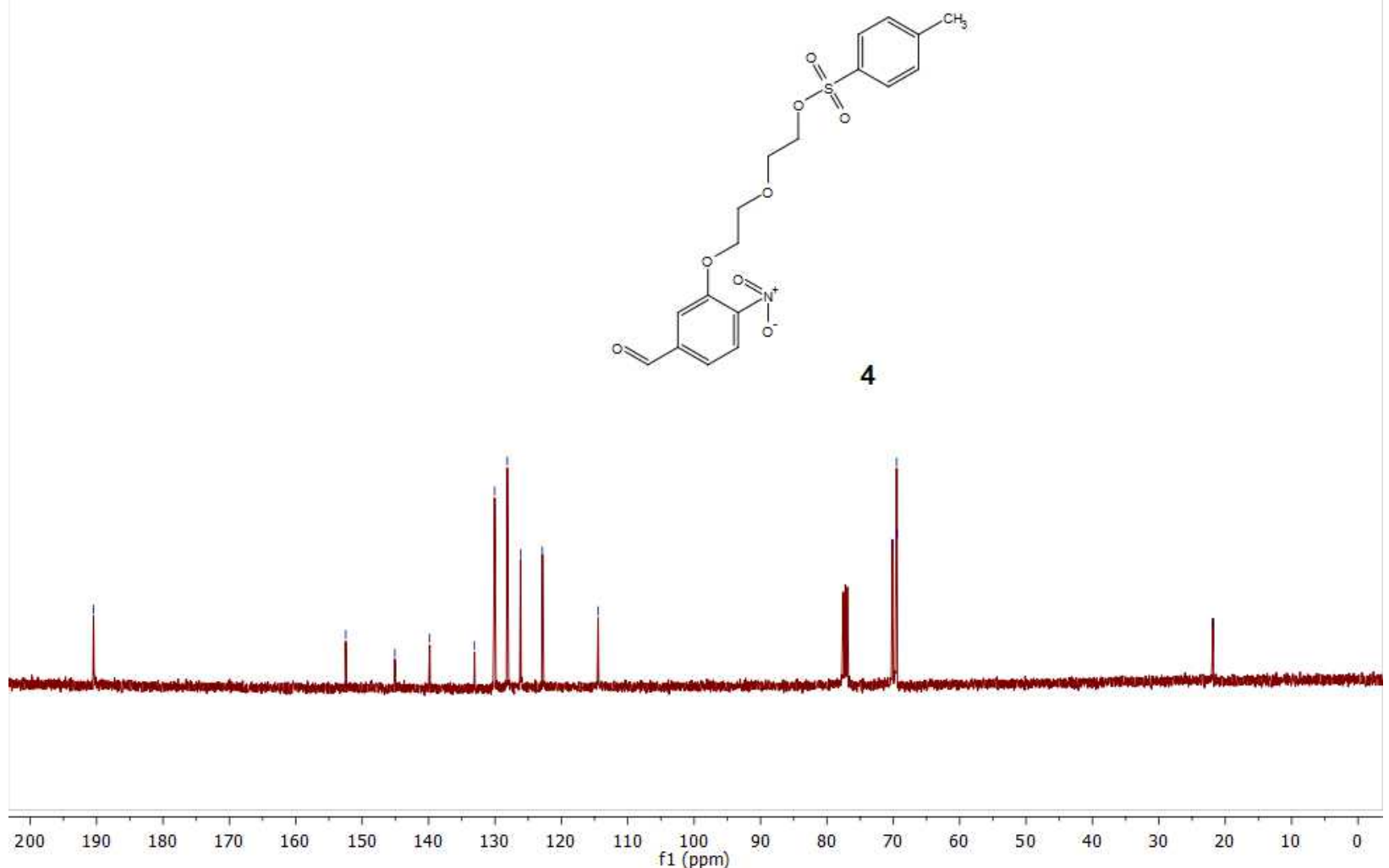


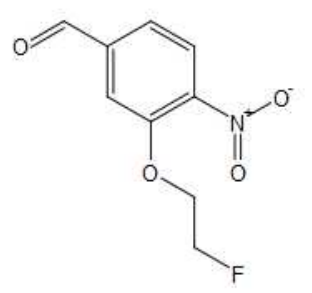

5

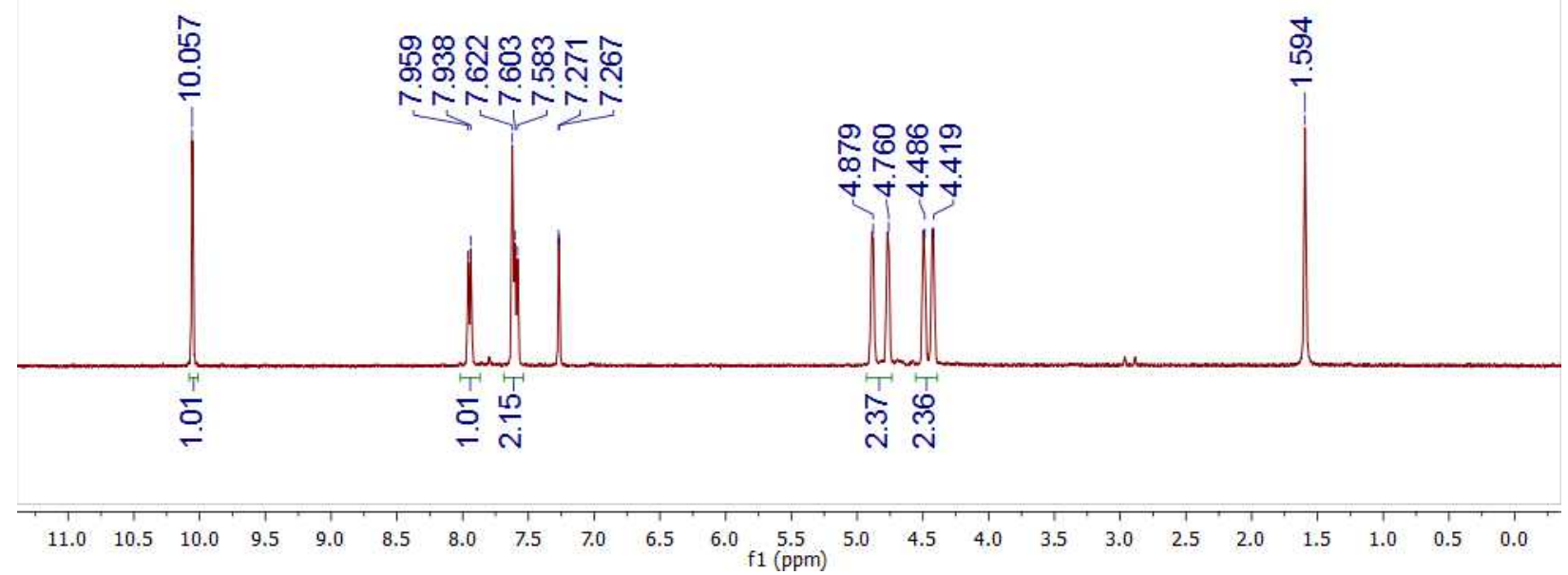




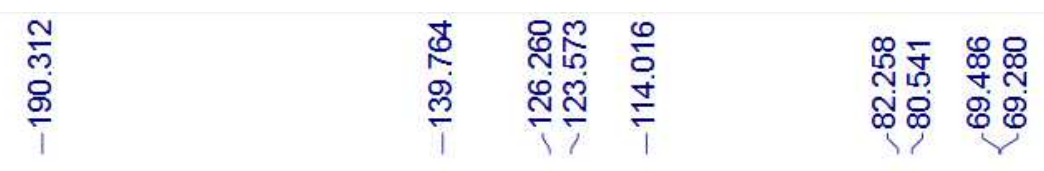

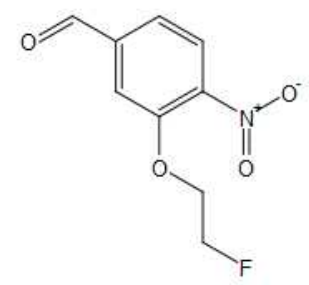

5
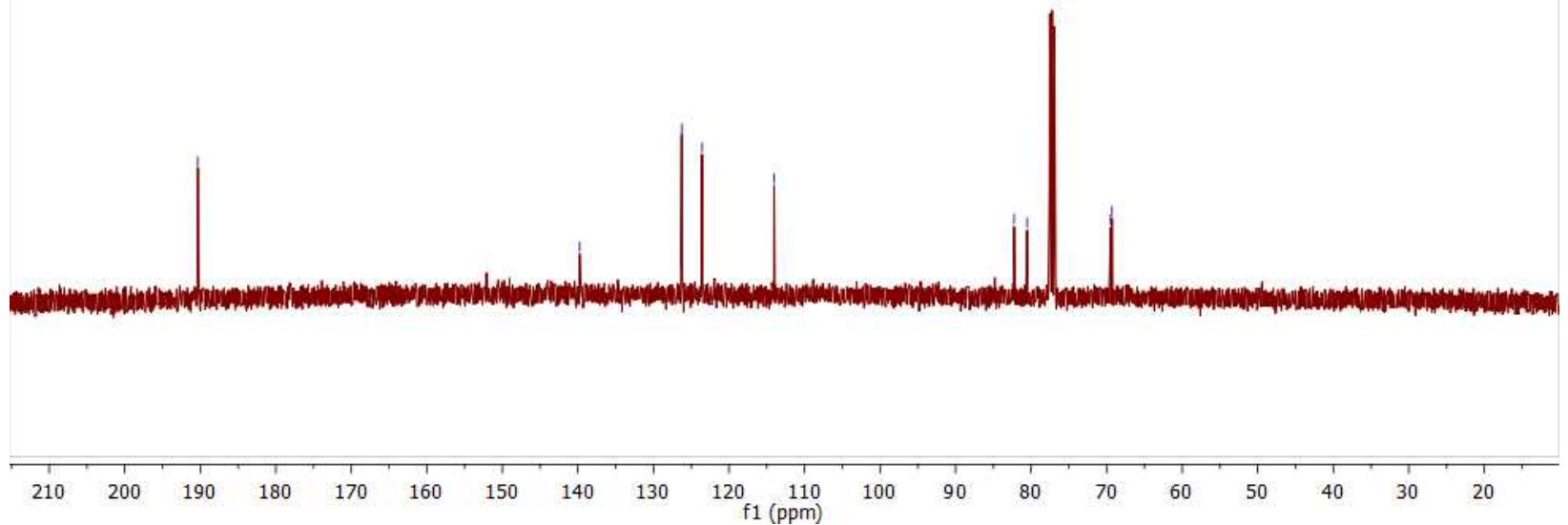


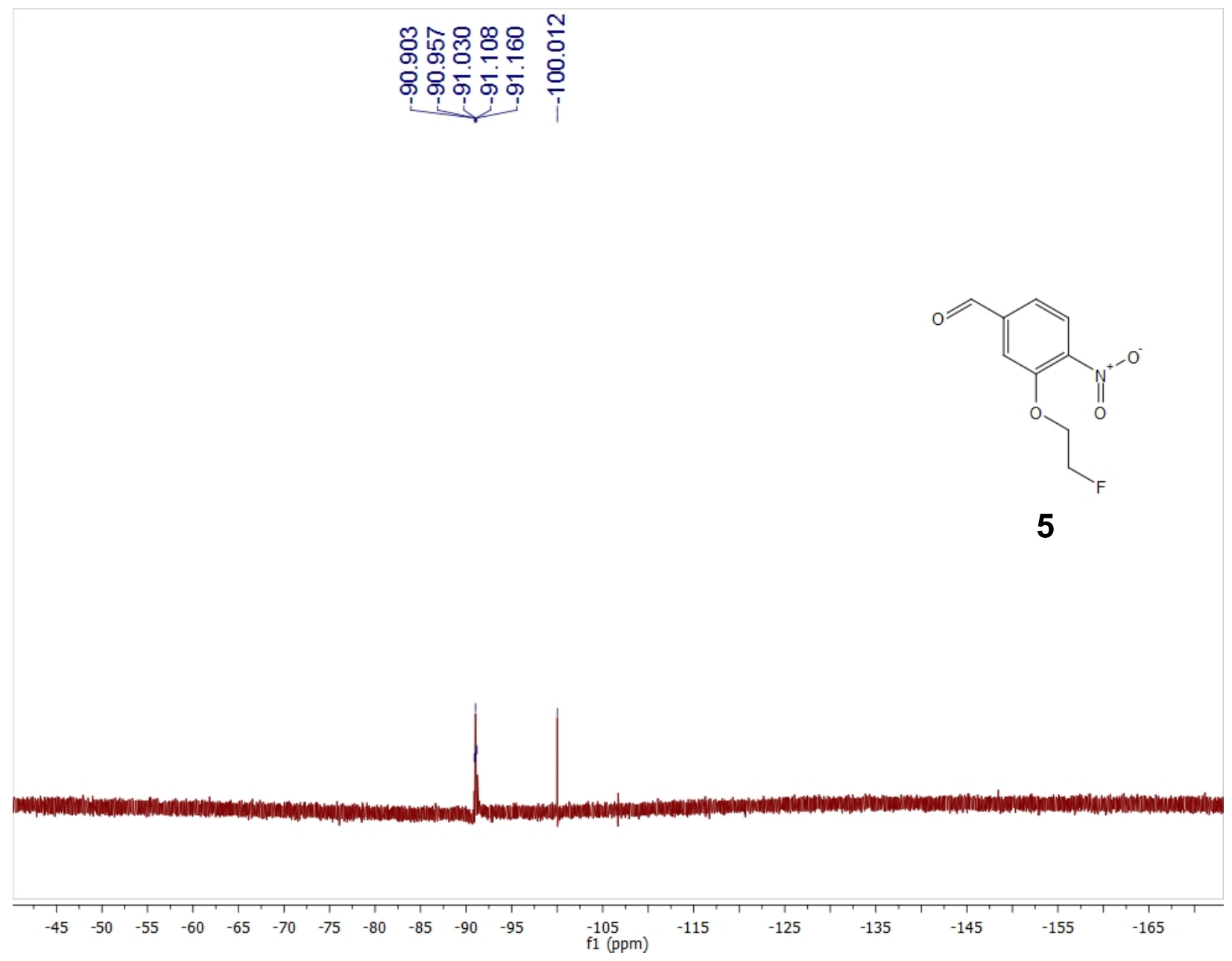



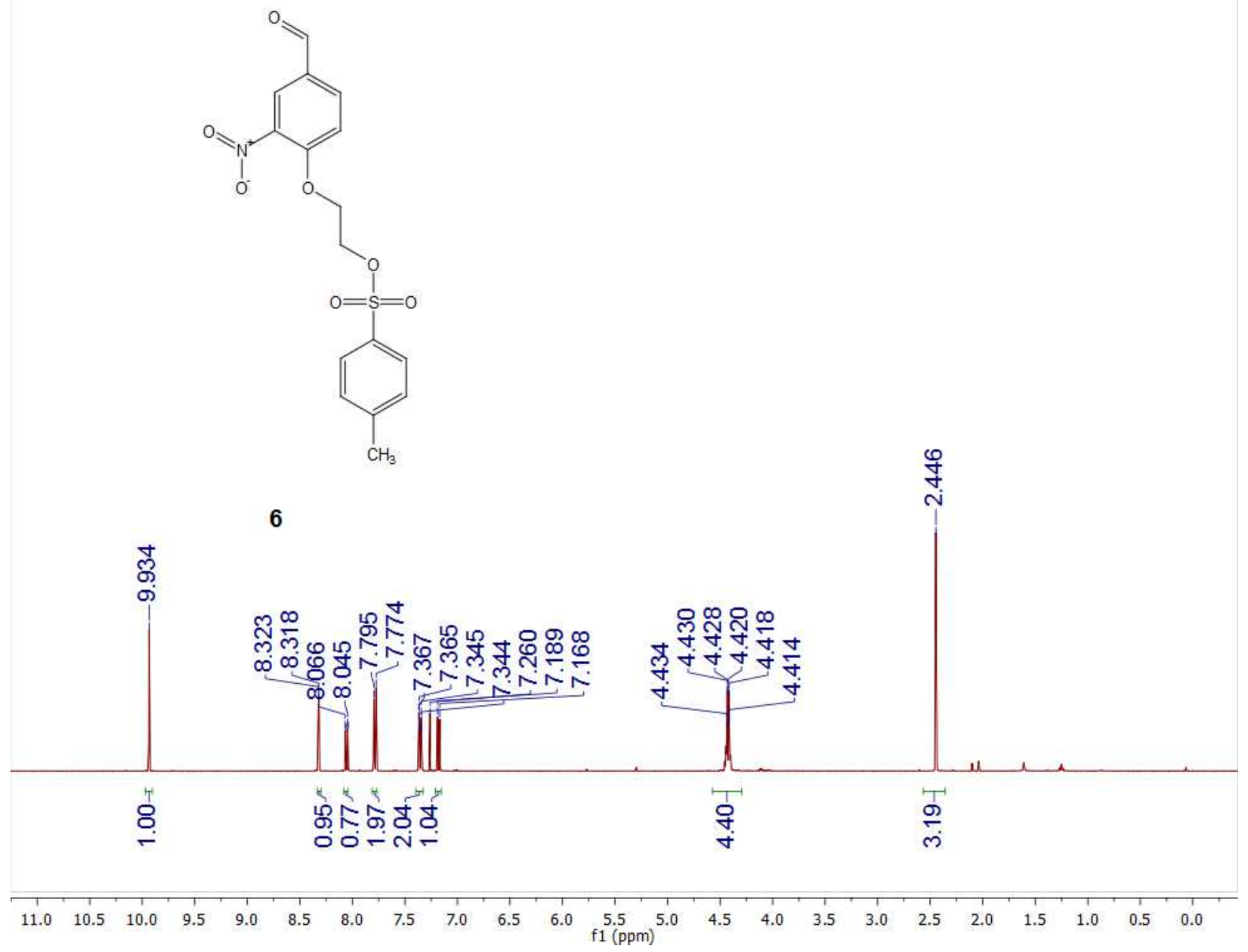


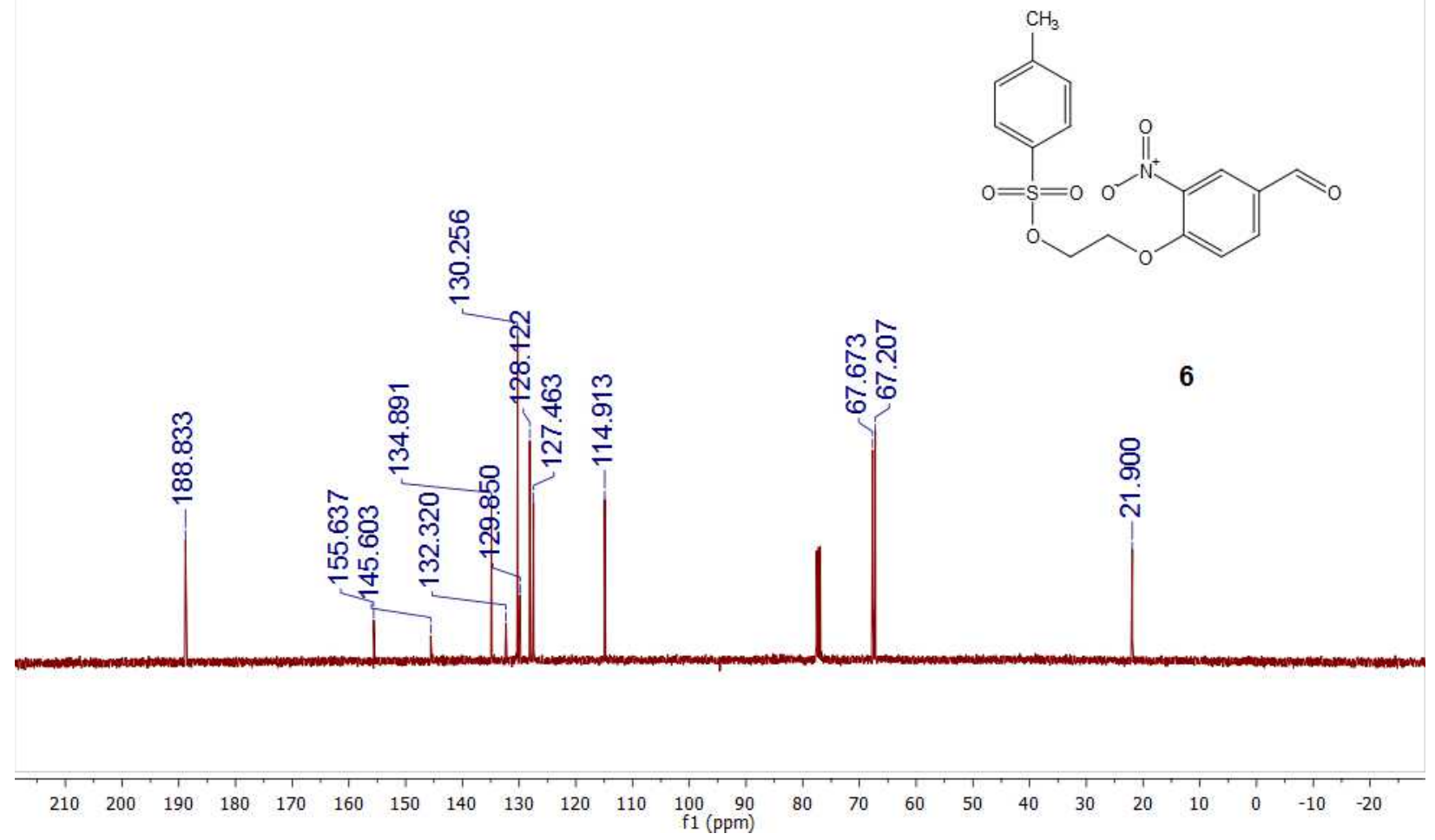




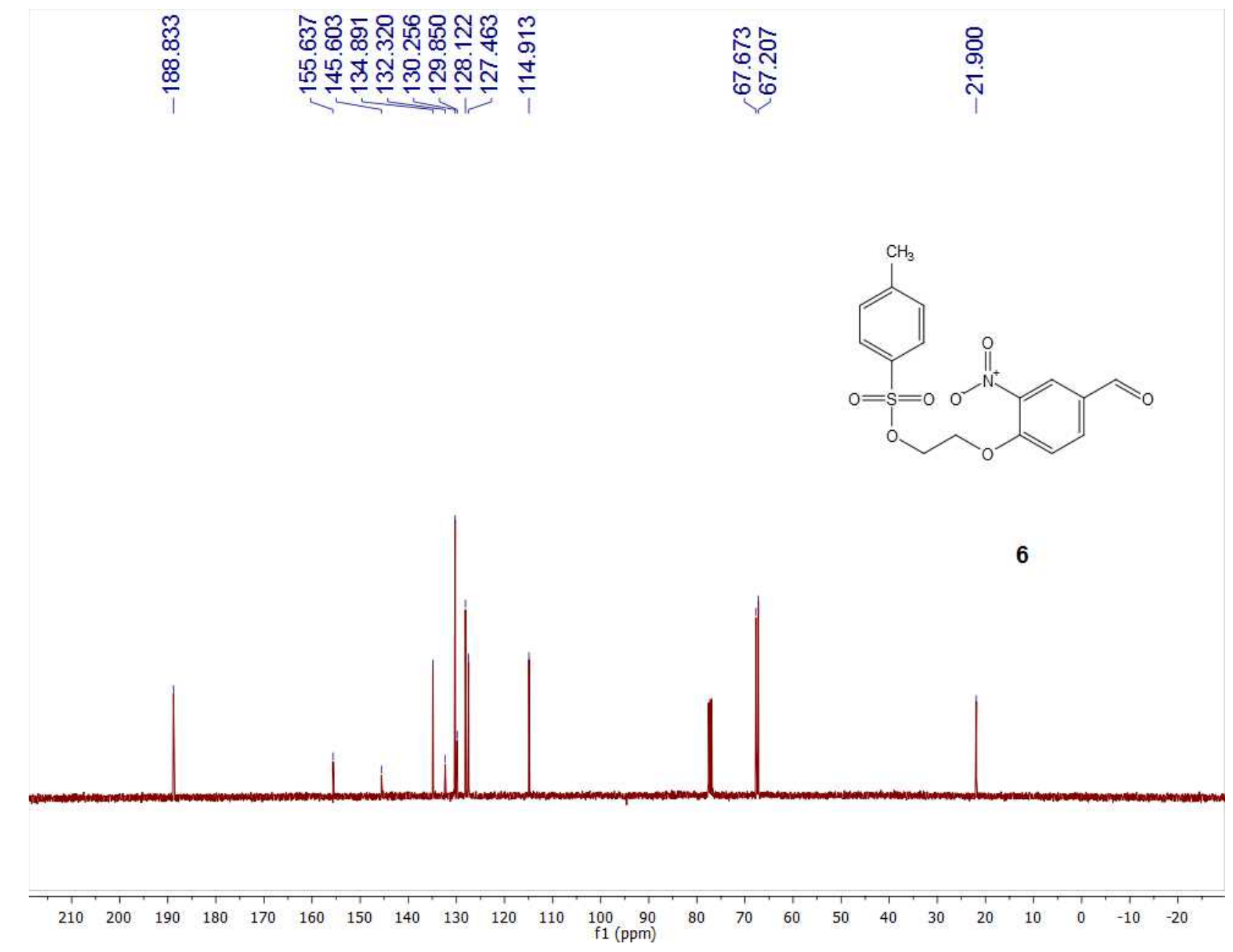



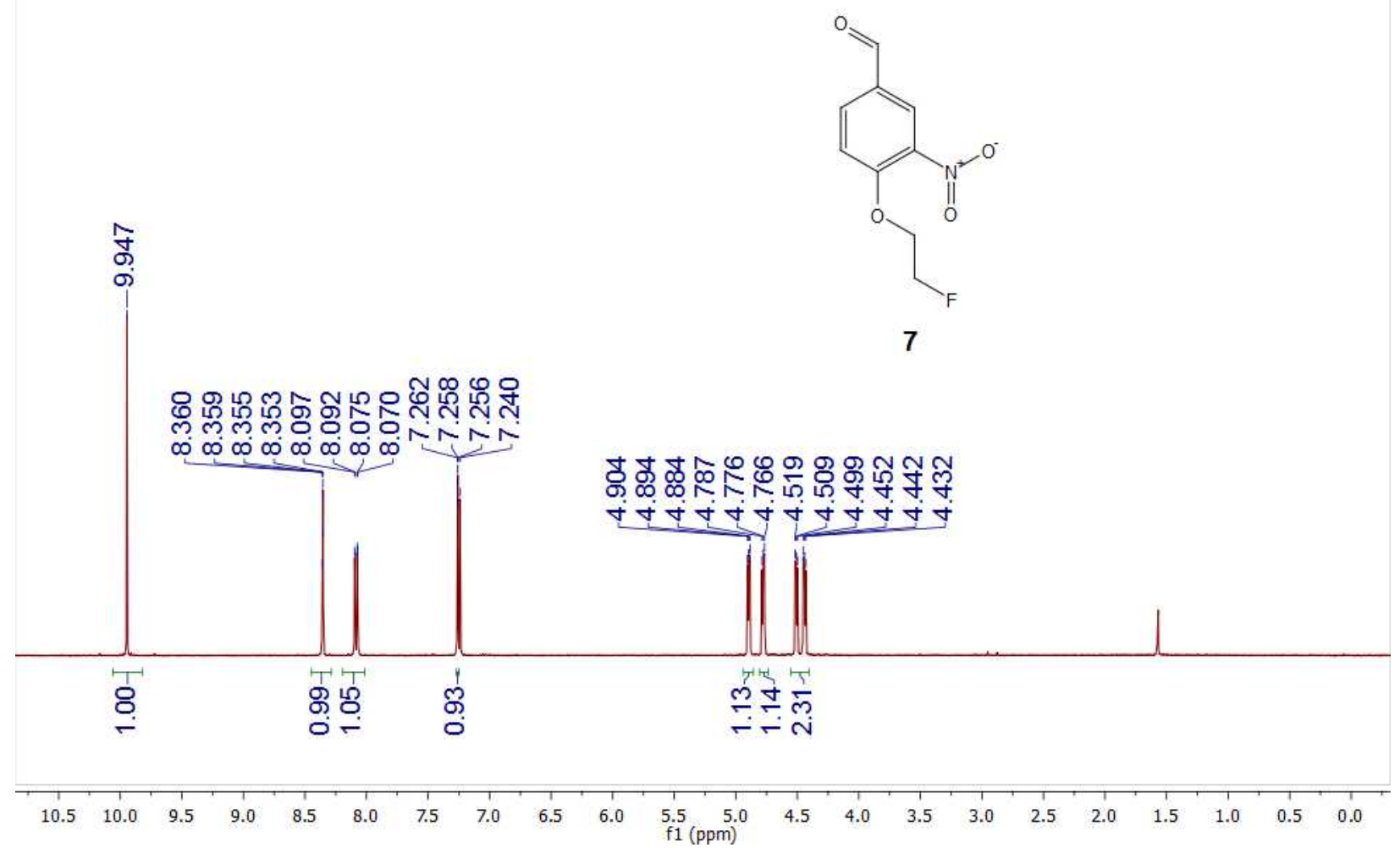


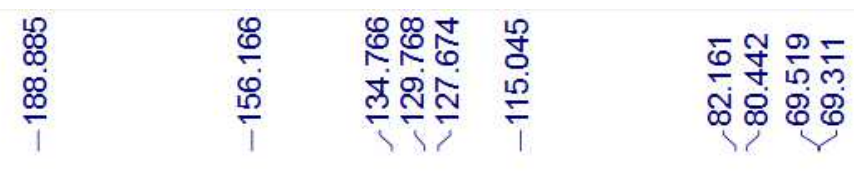

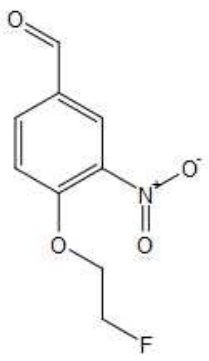

7
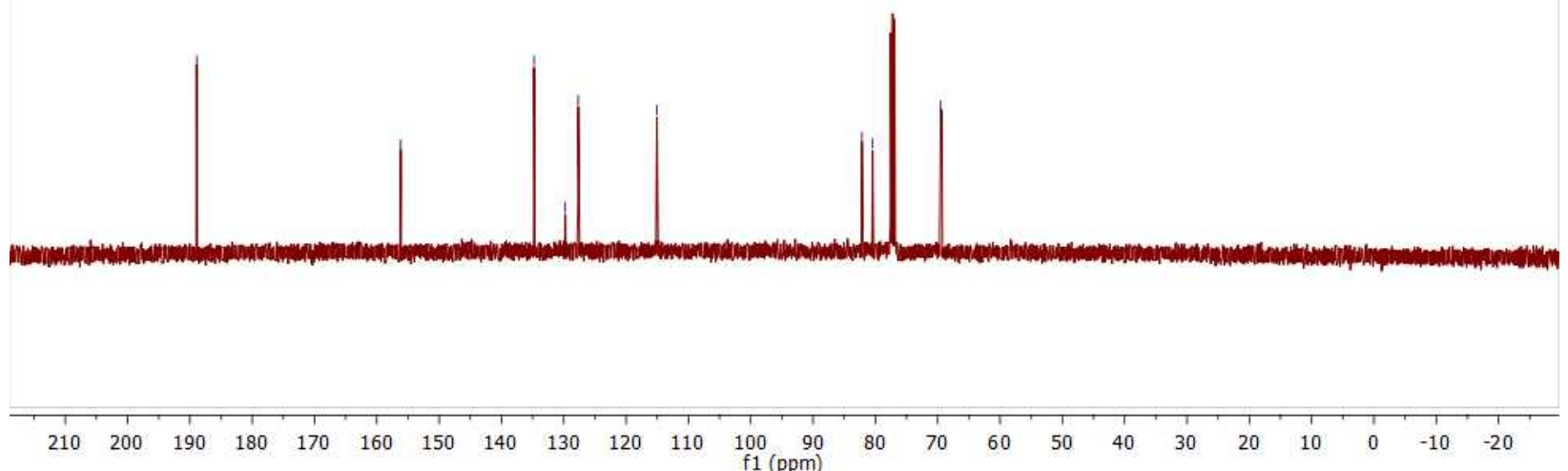


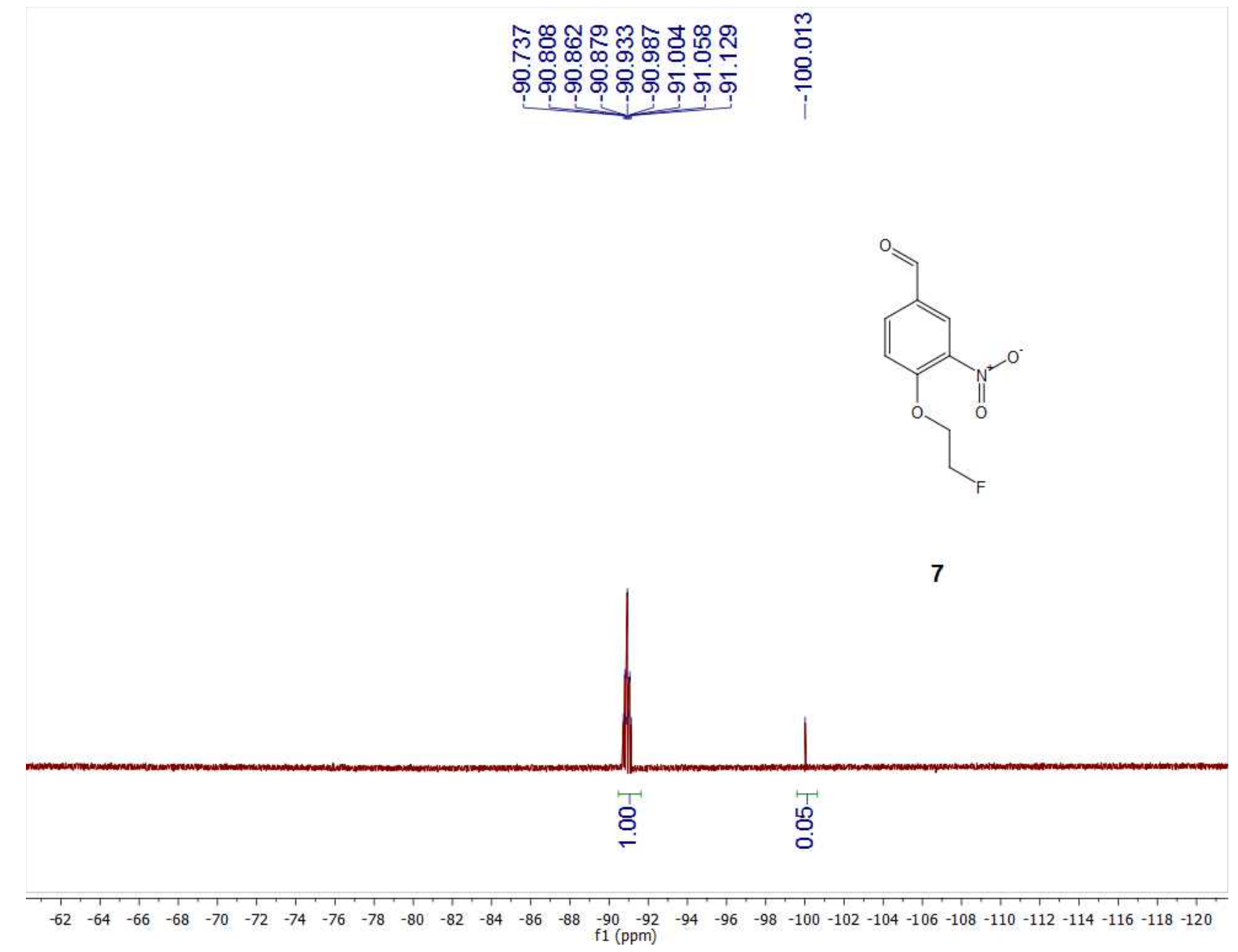




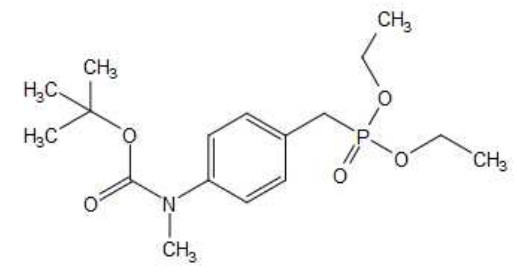

10

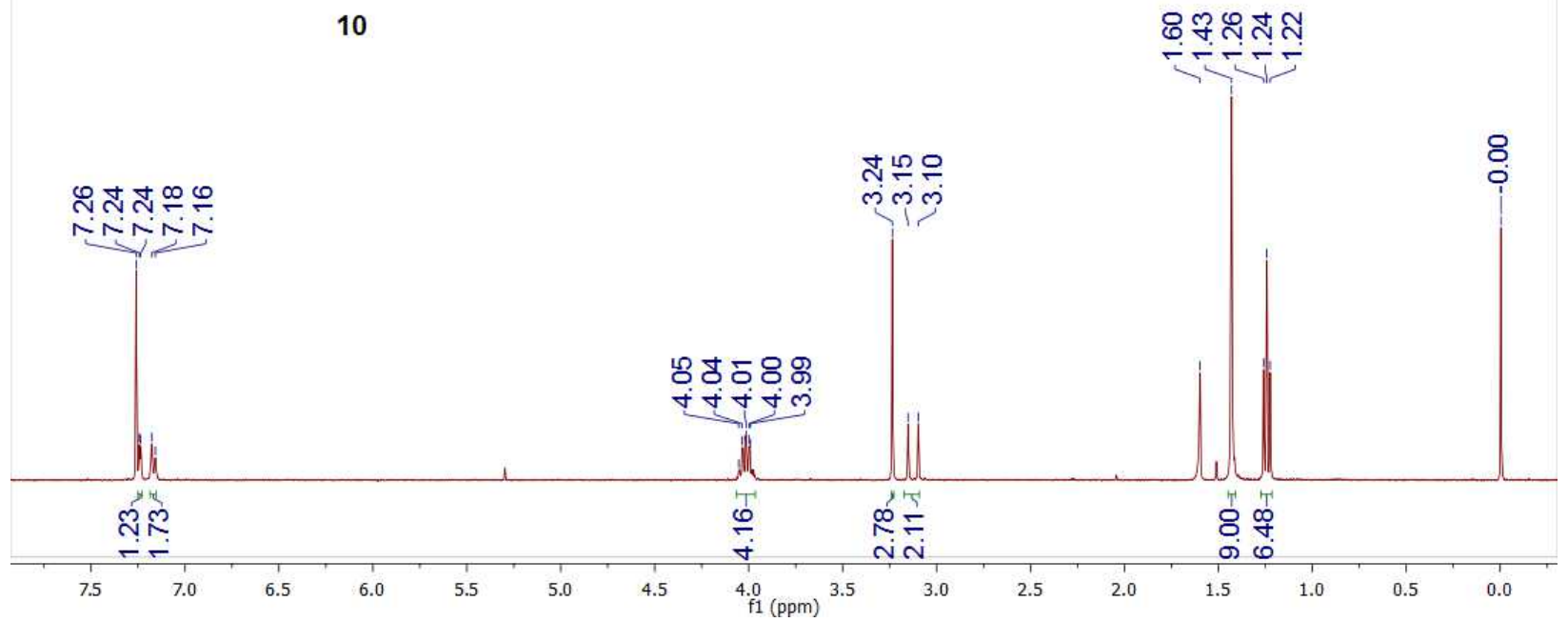




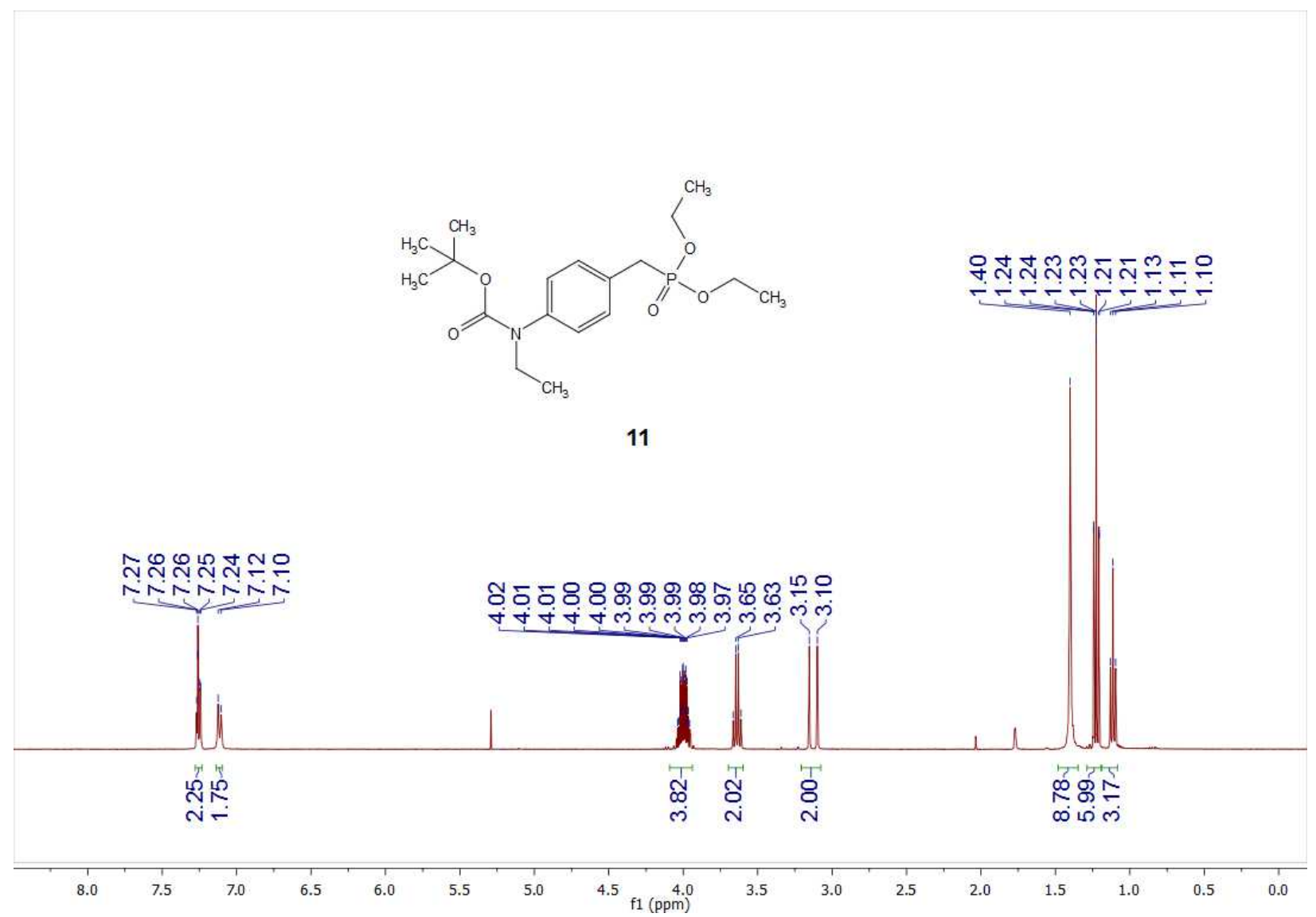




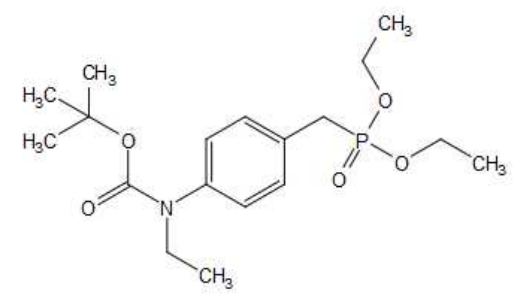

11

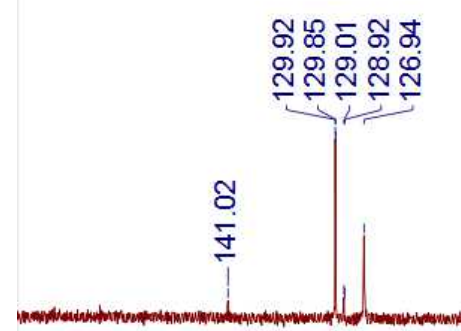

욤

లెల్లి

1)

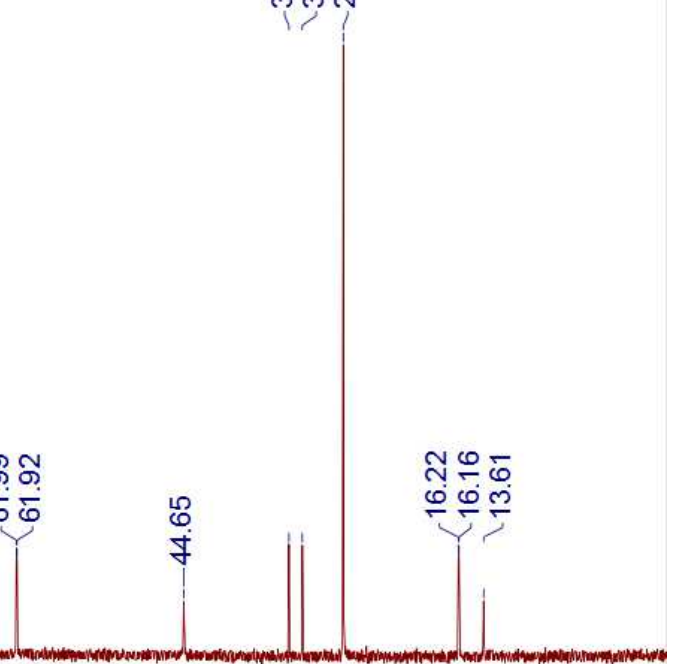

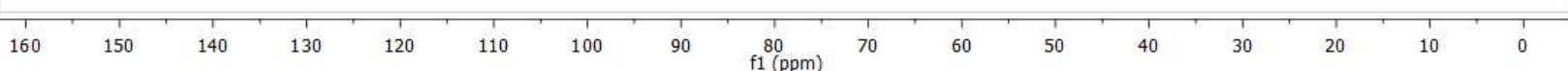



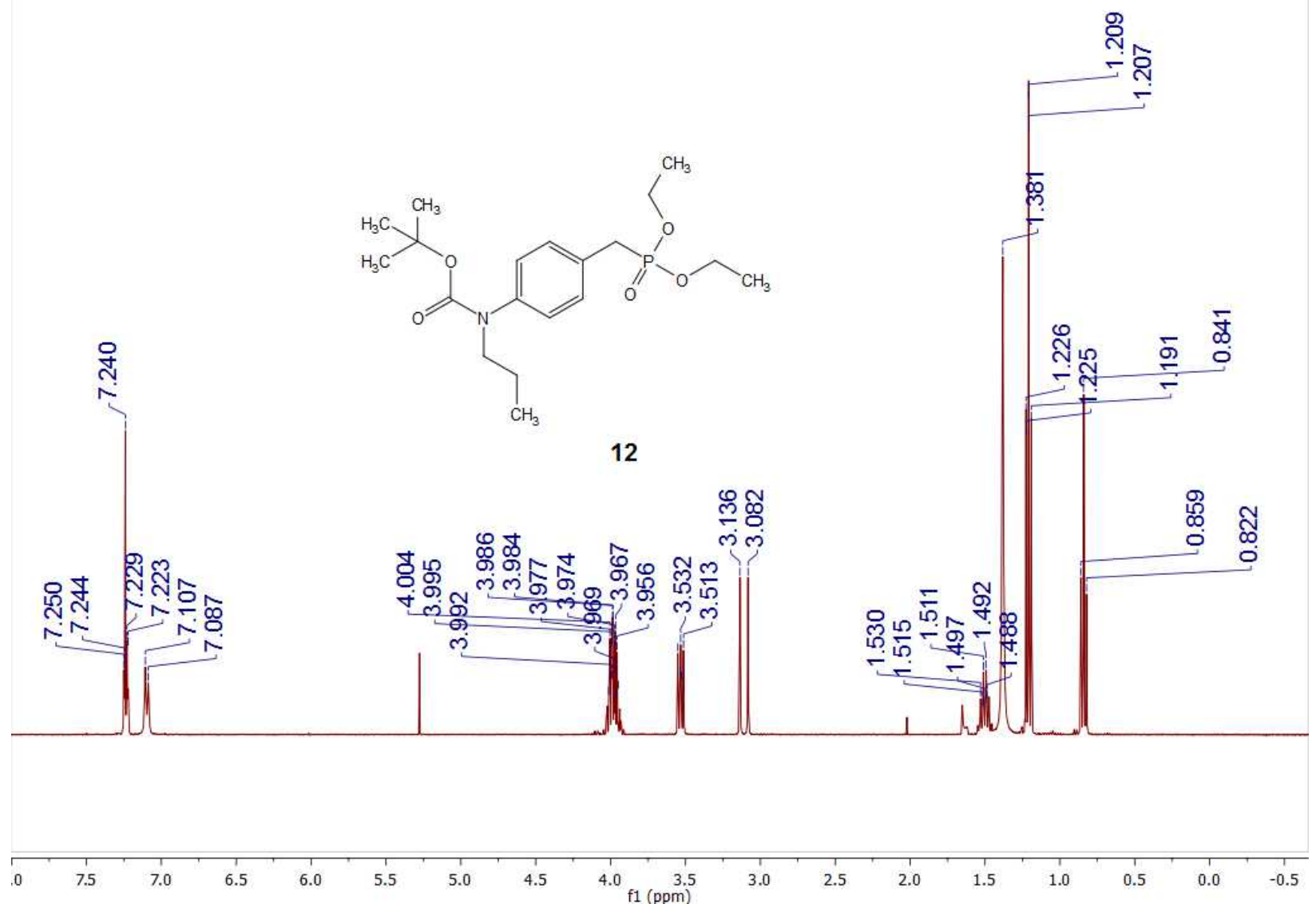


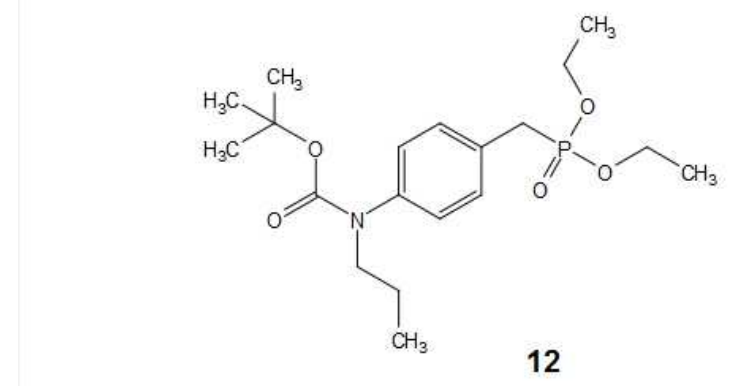

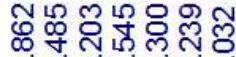

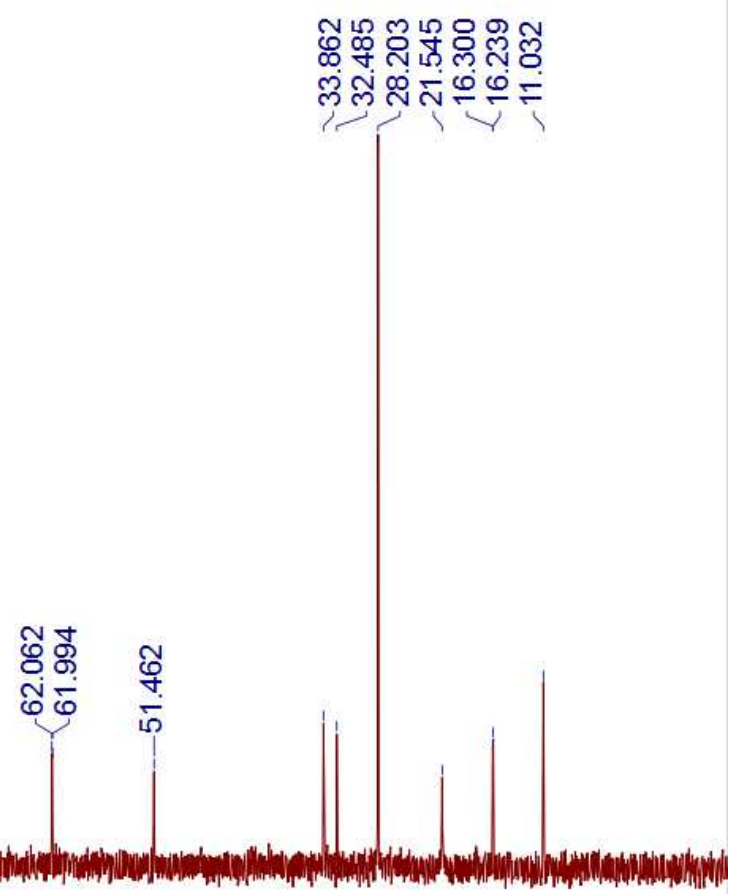

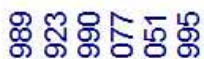

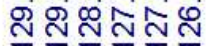
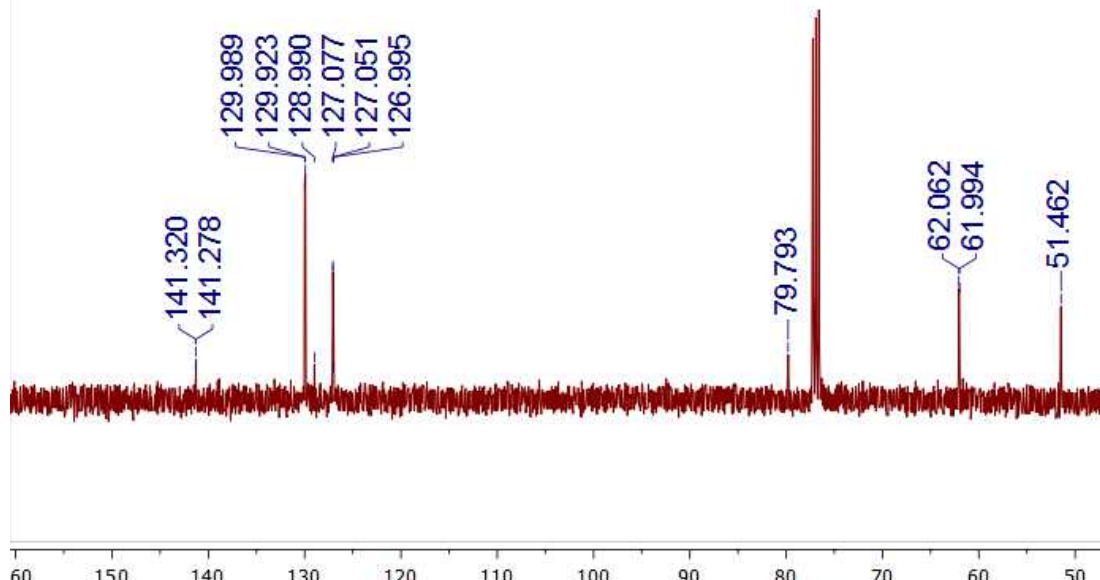

80 f1 (ppm) 


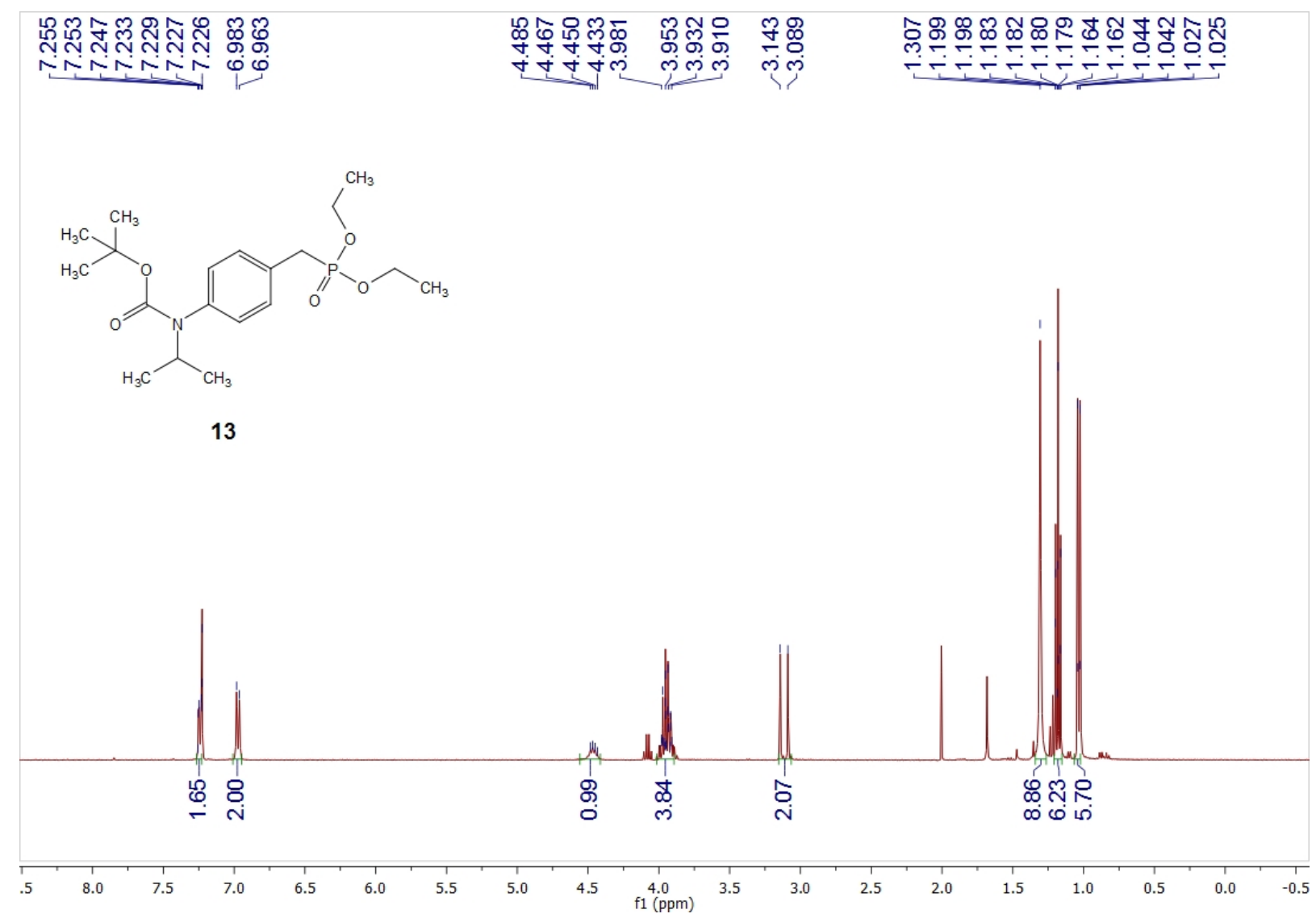




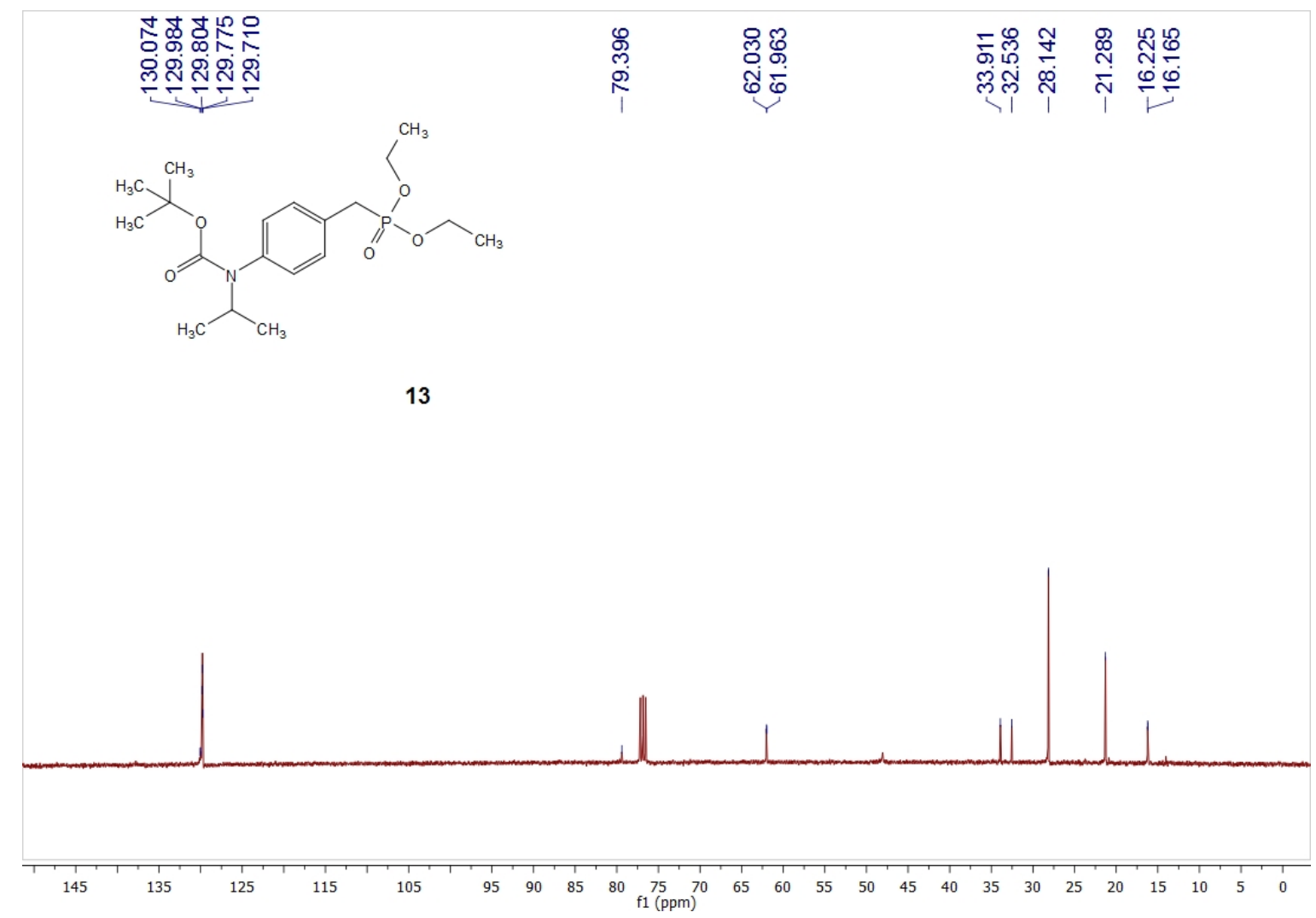




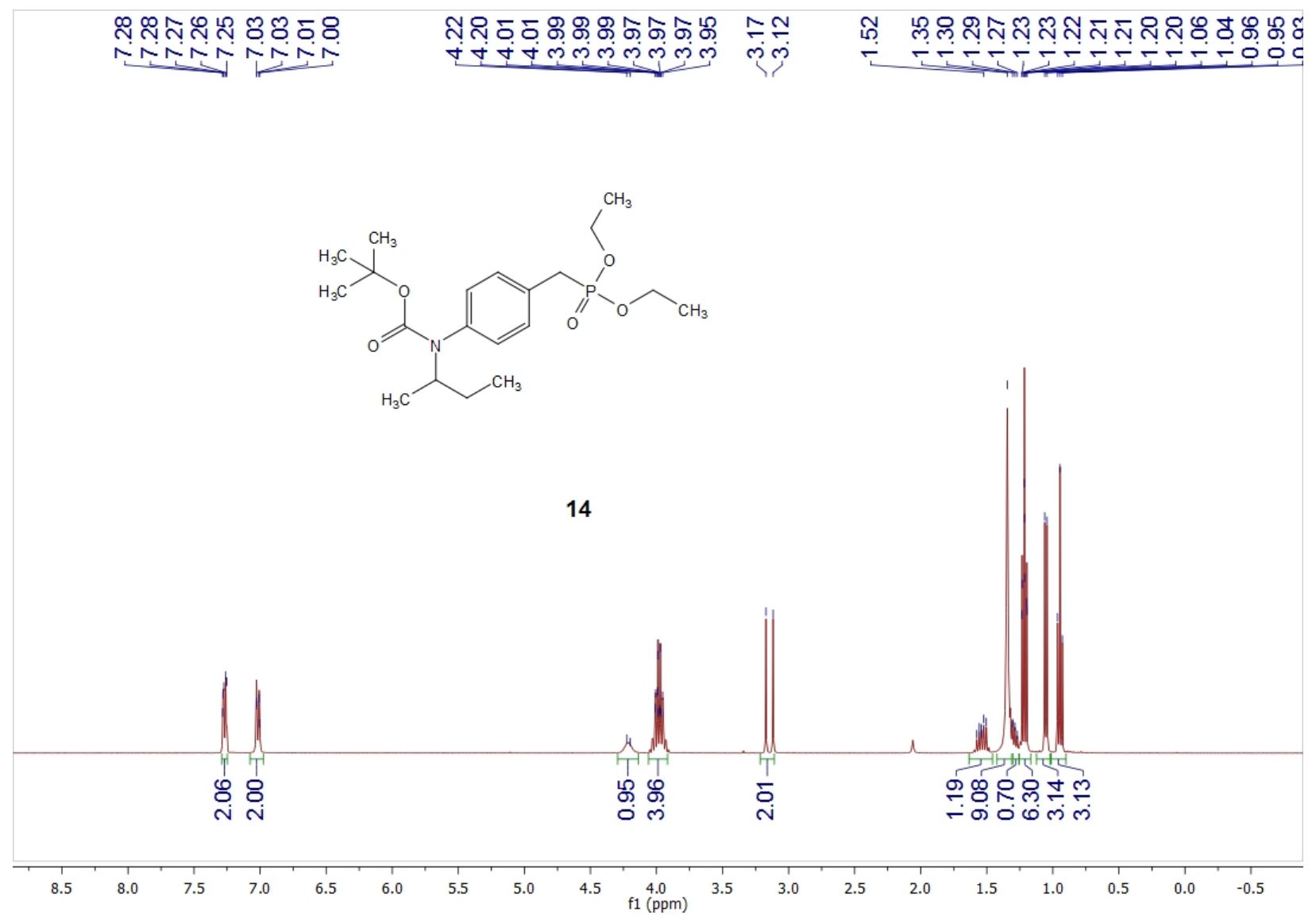




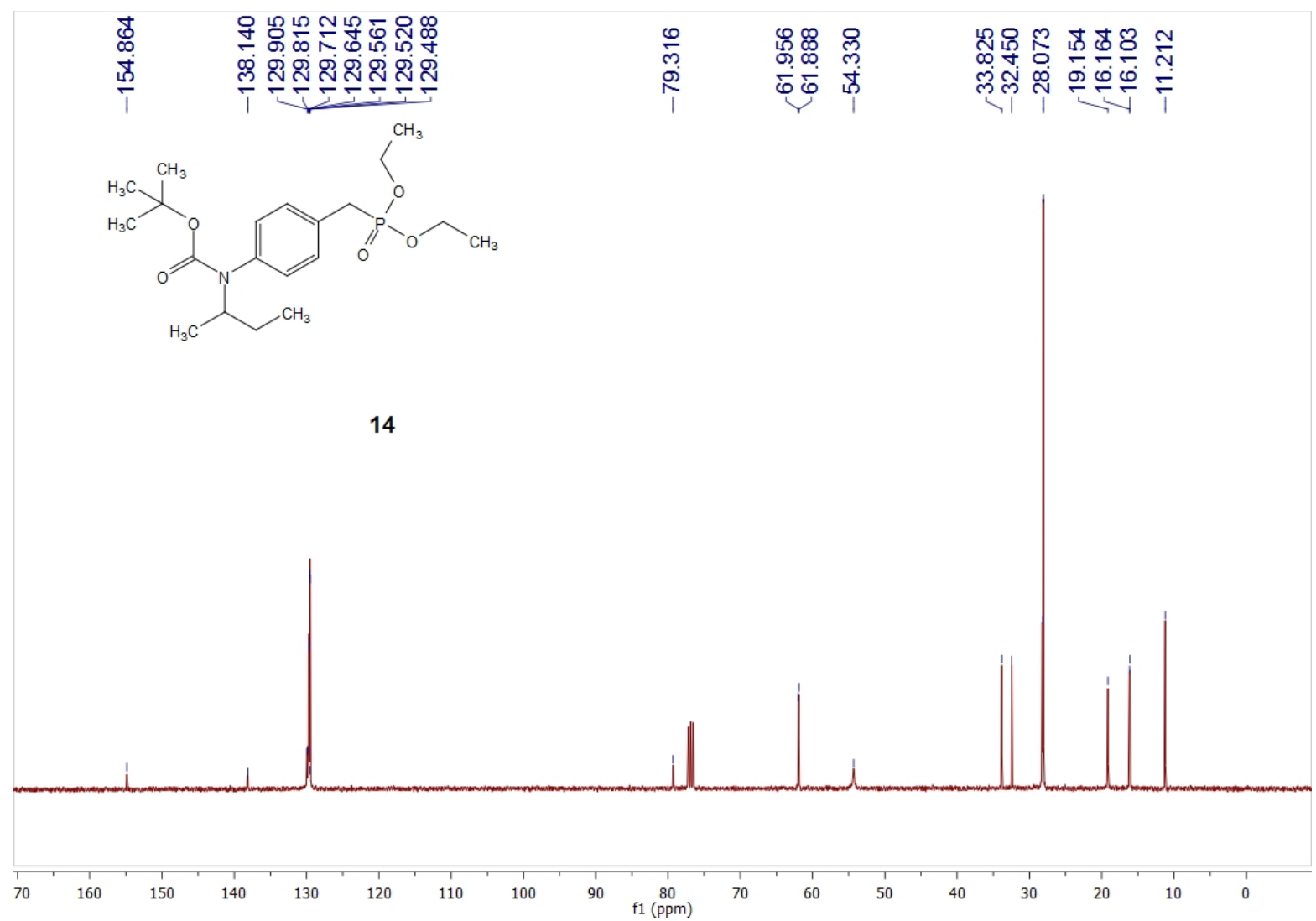




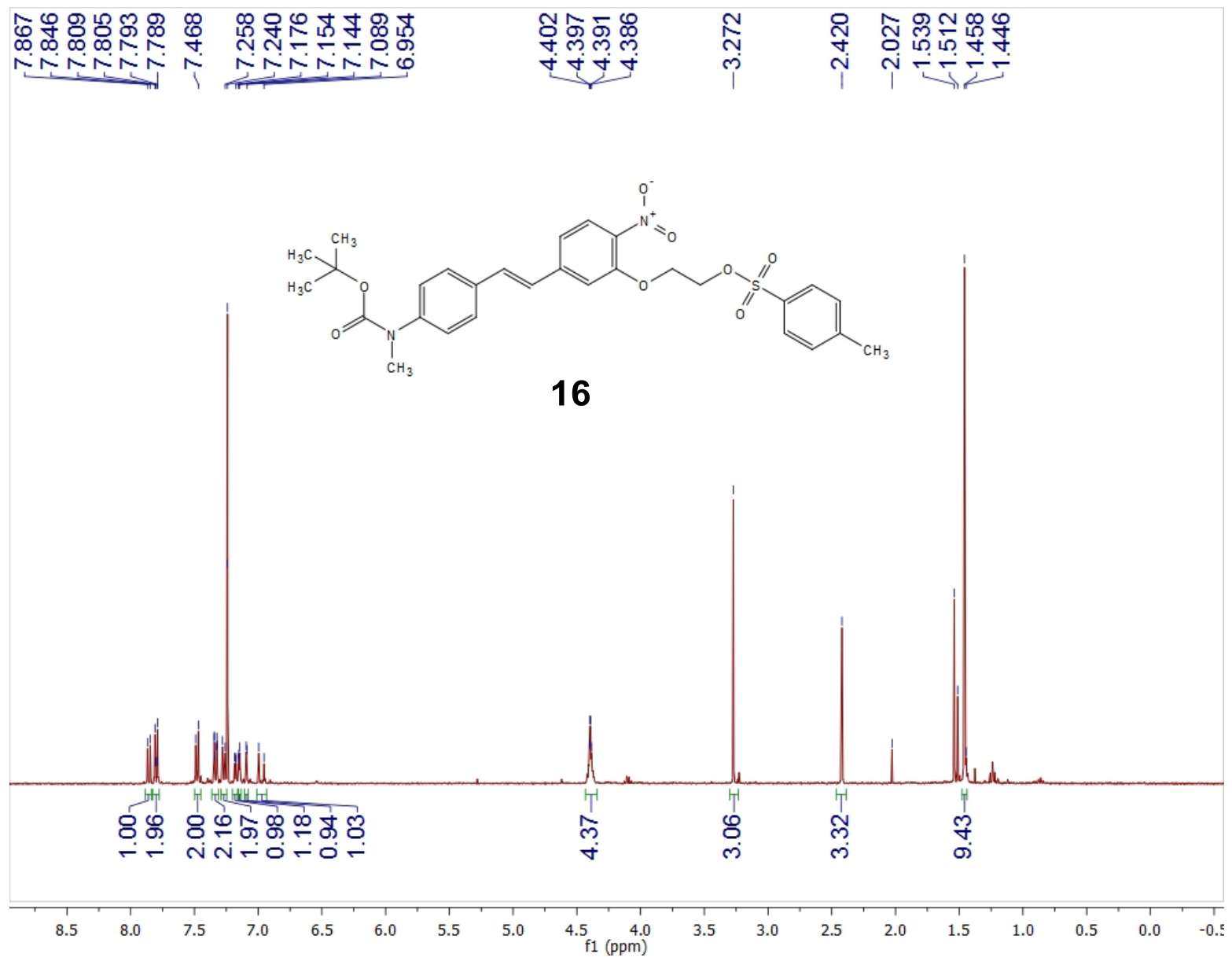




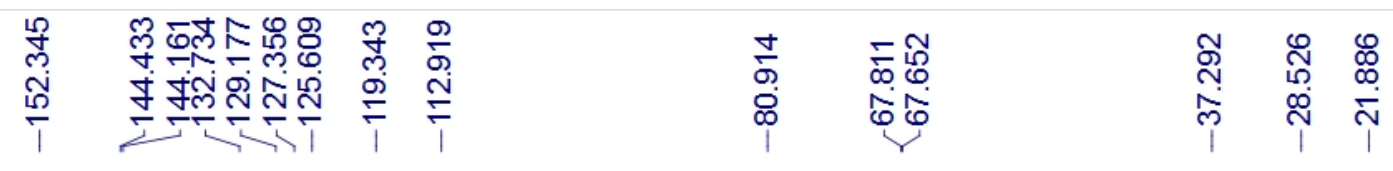

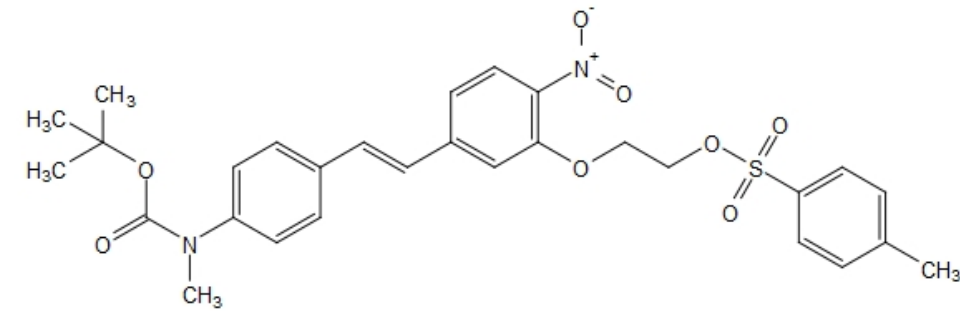

16
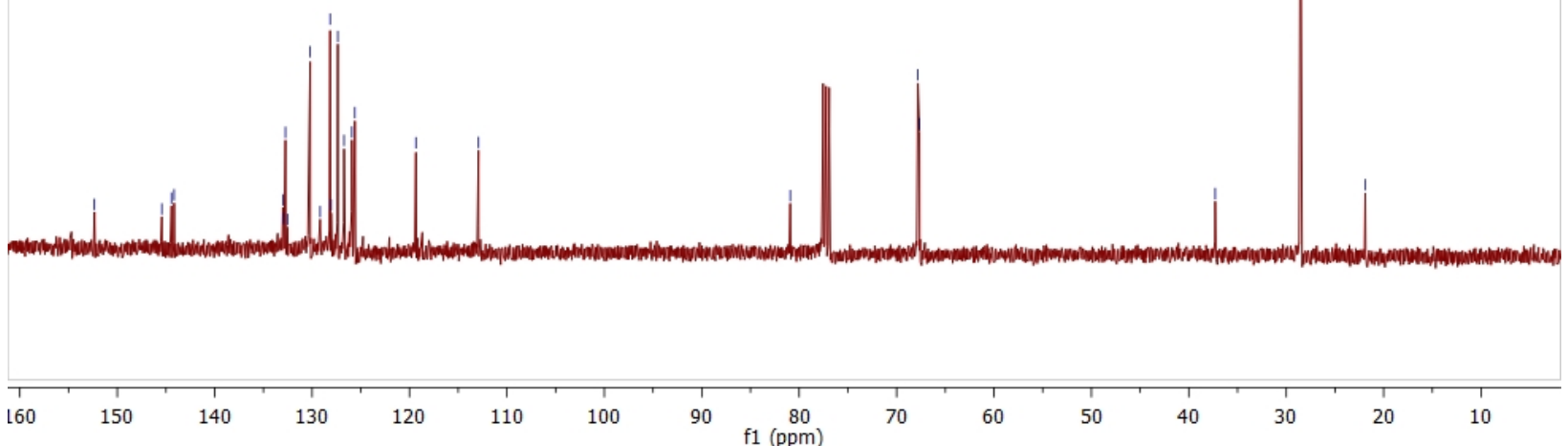


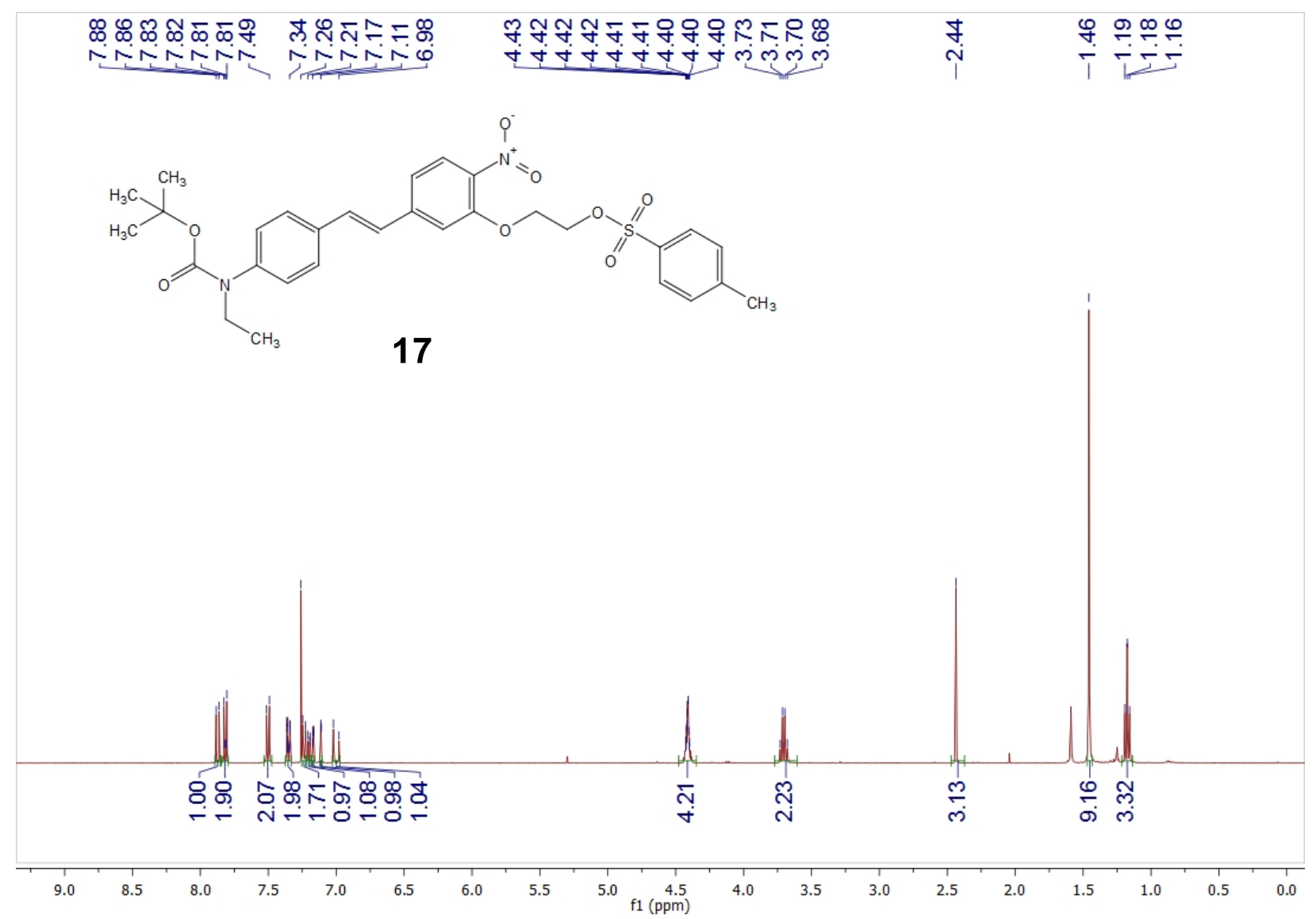




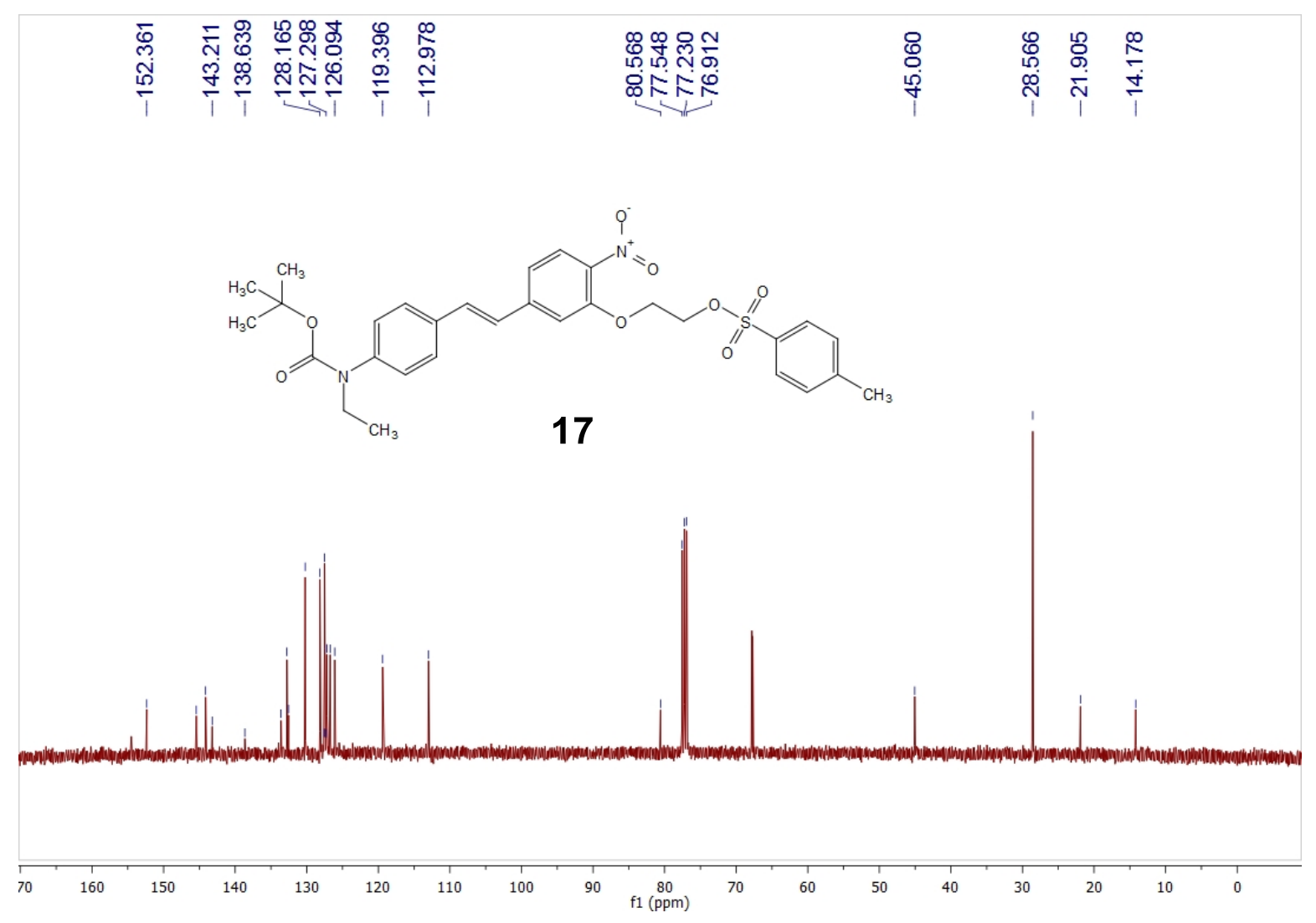




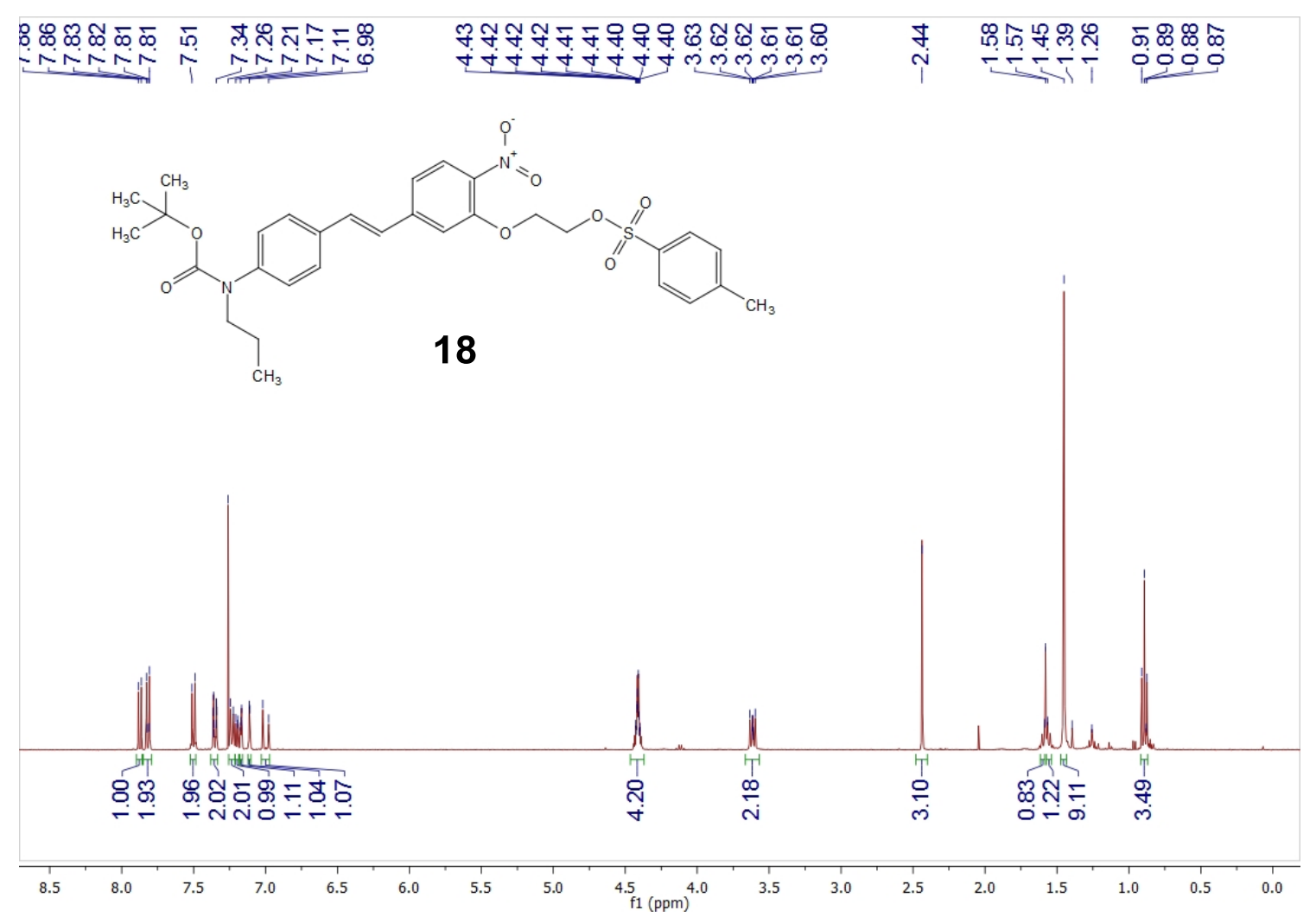




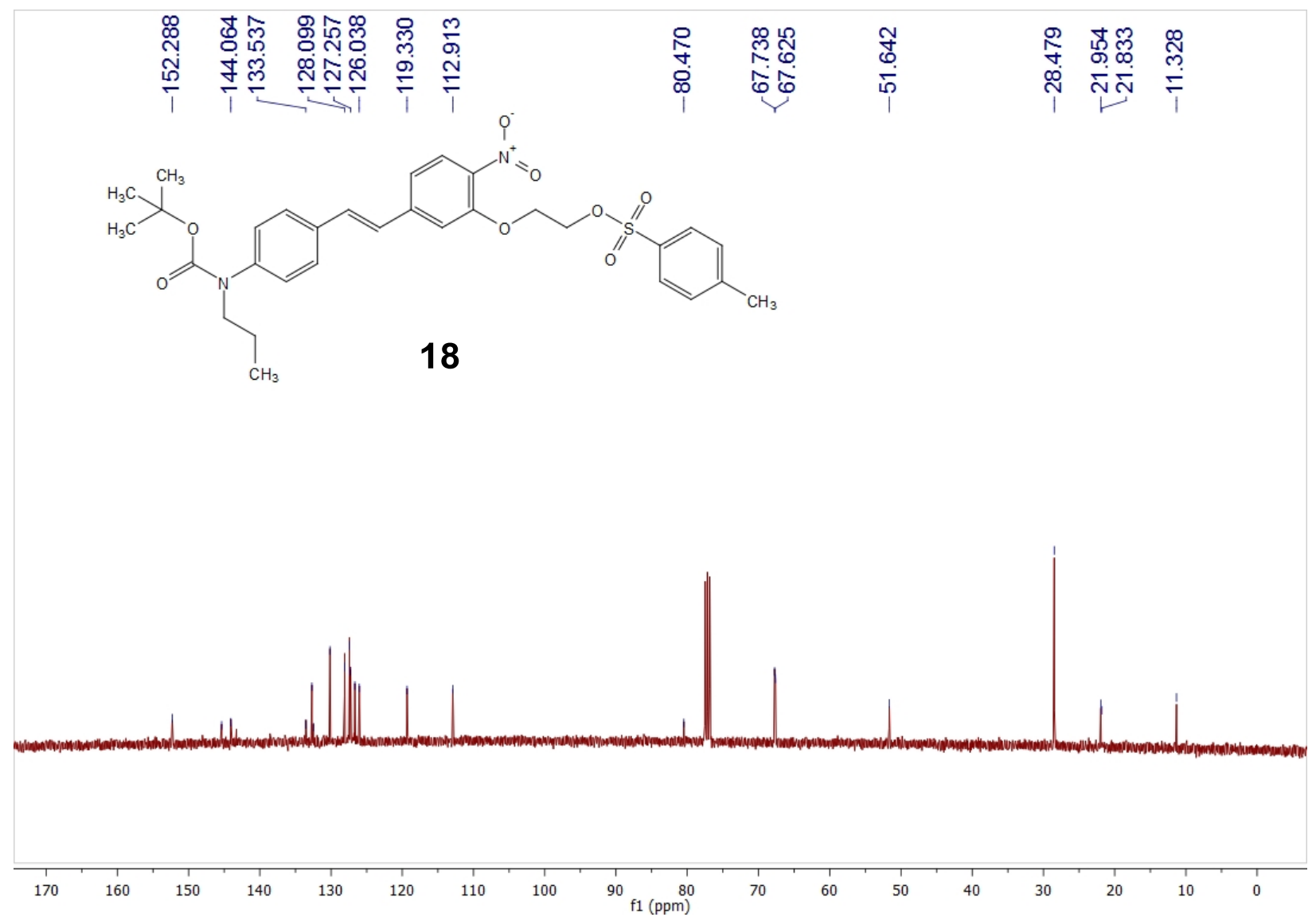




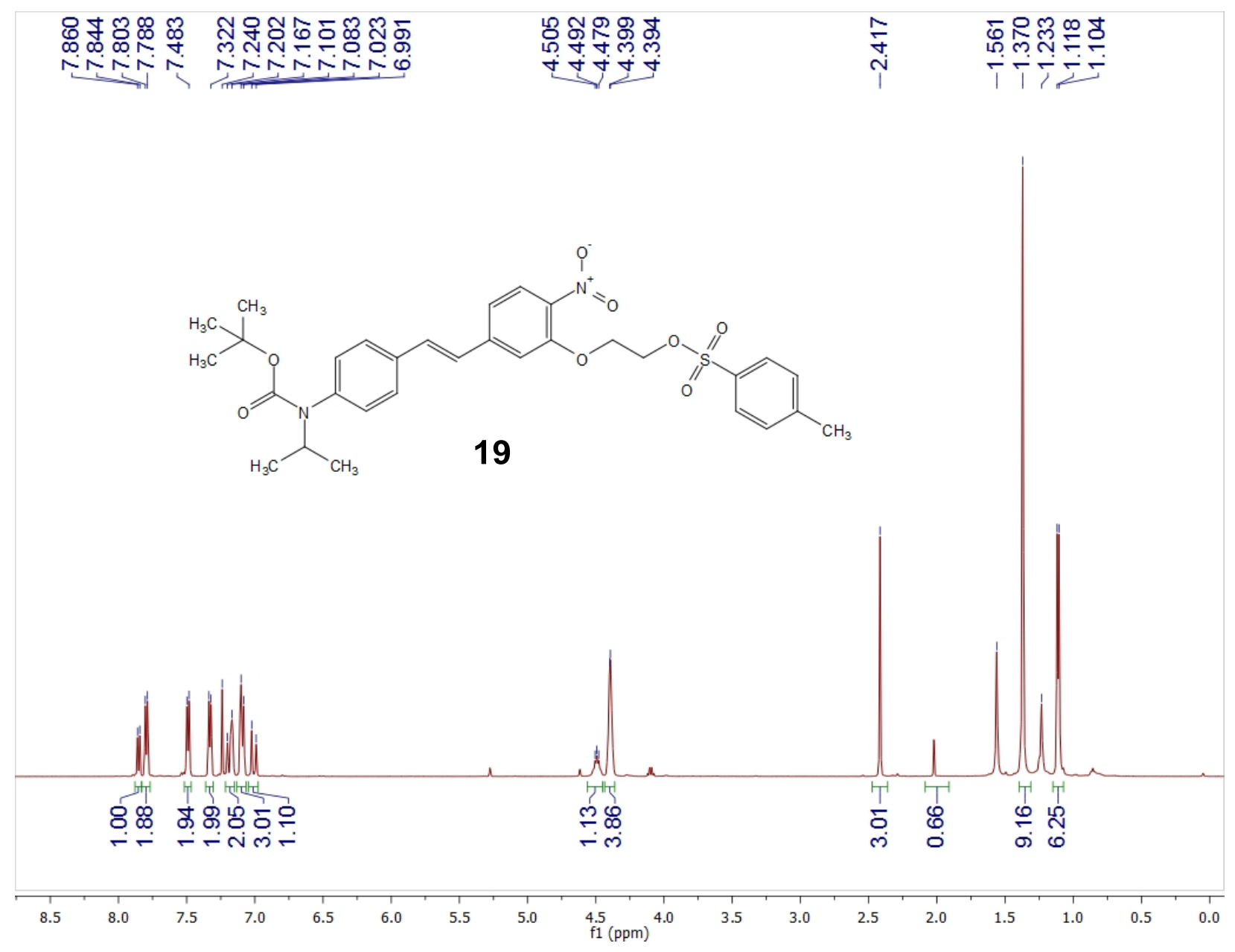




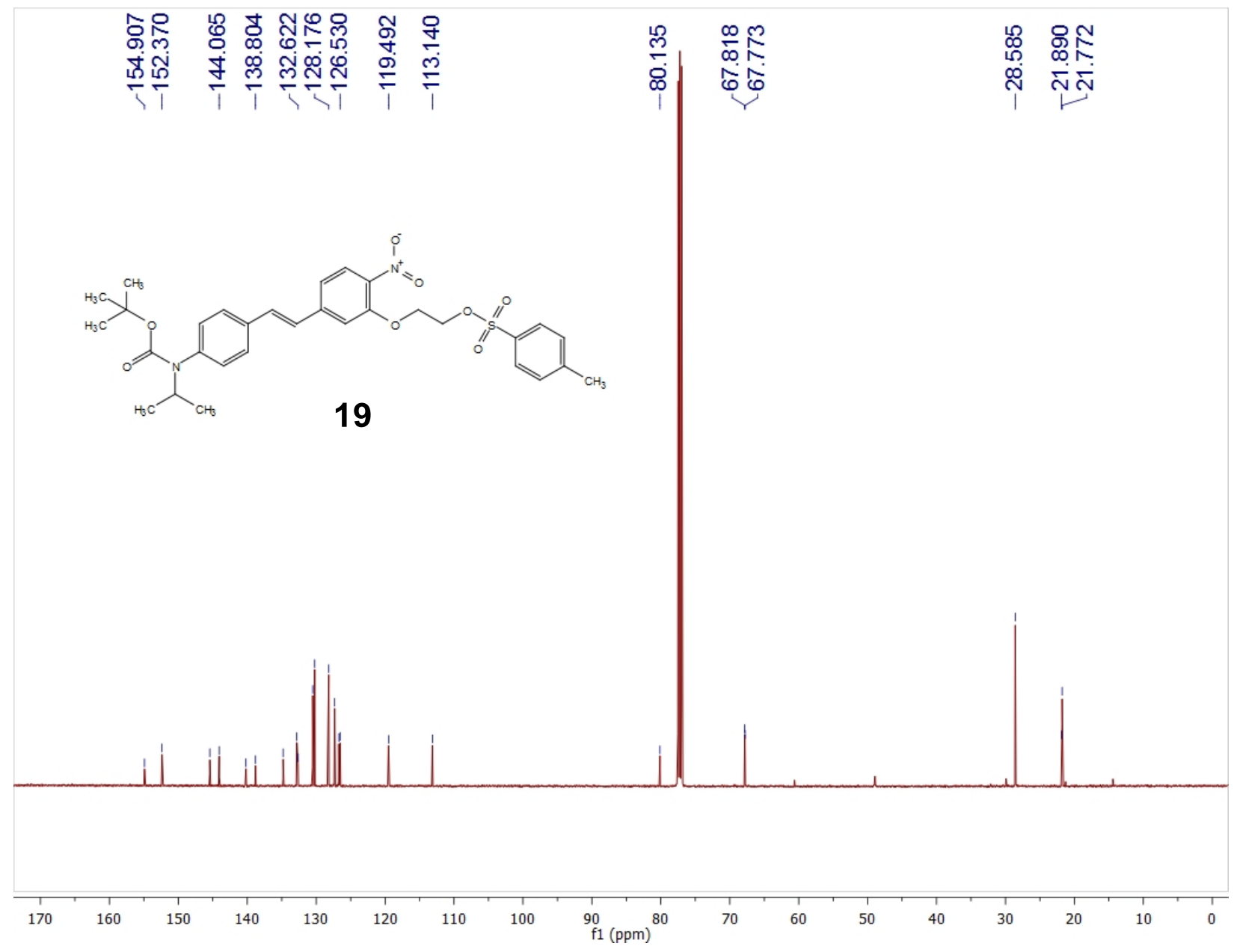




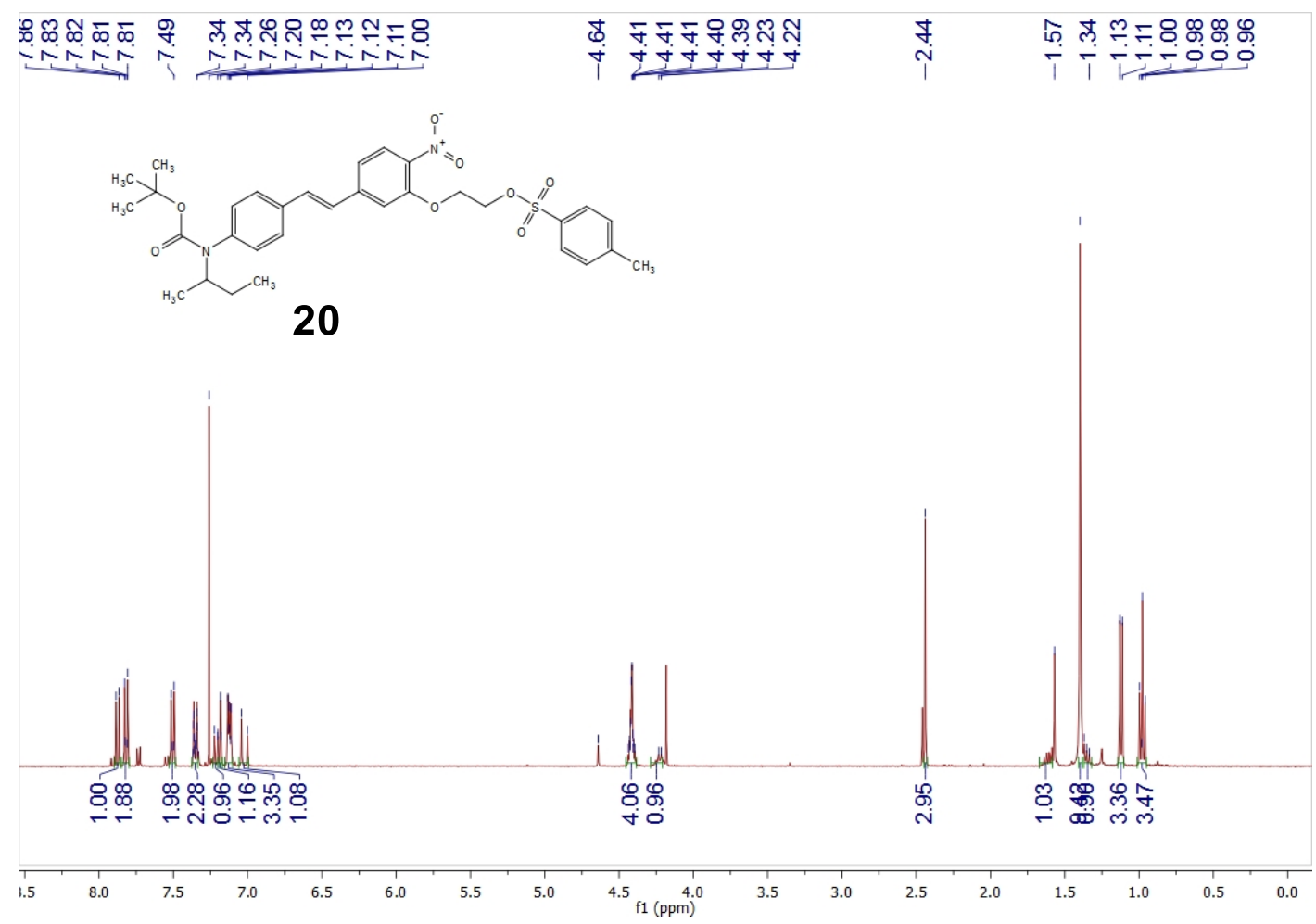




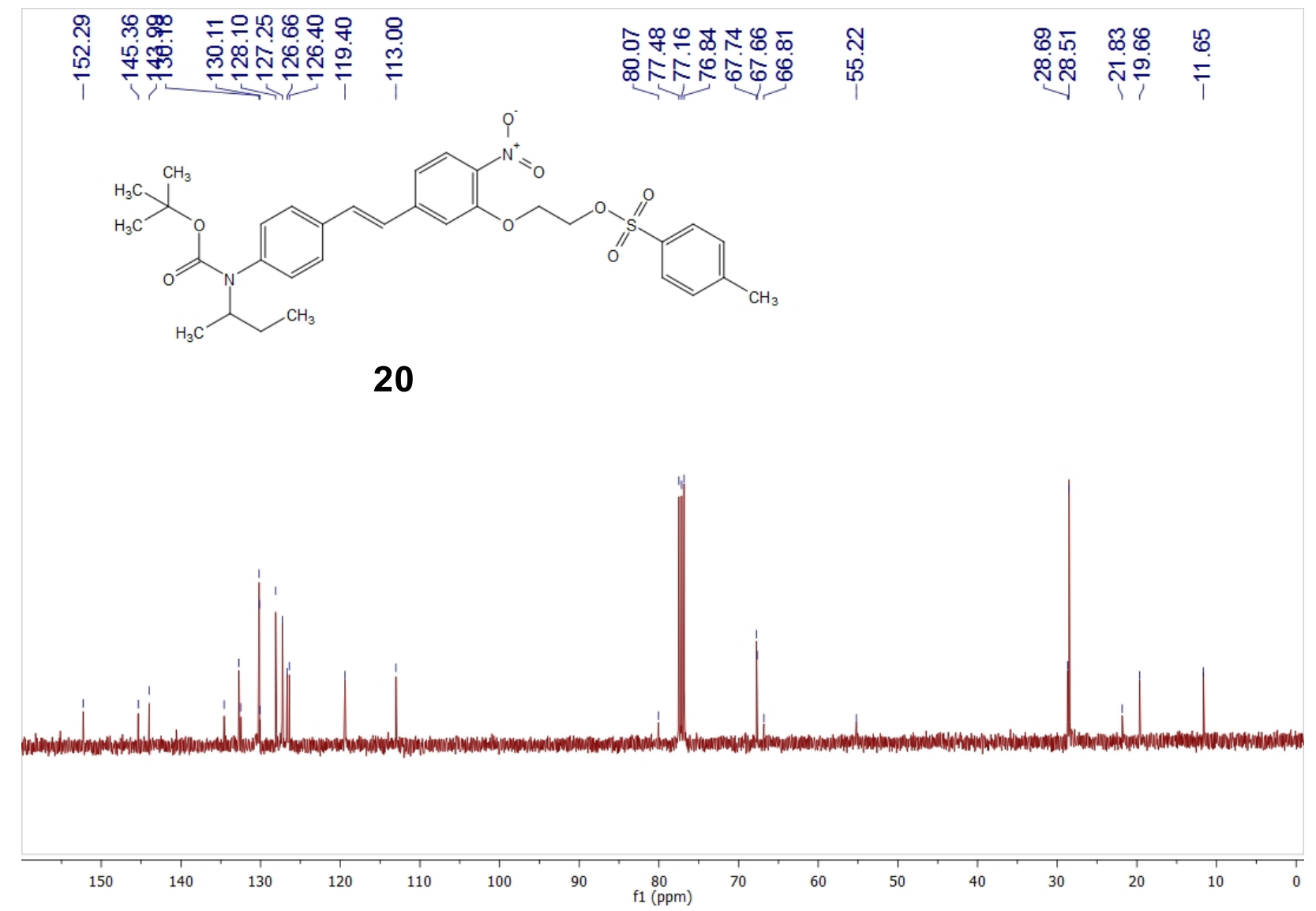




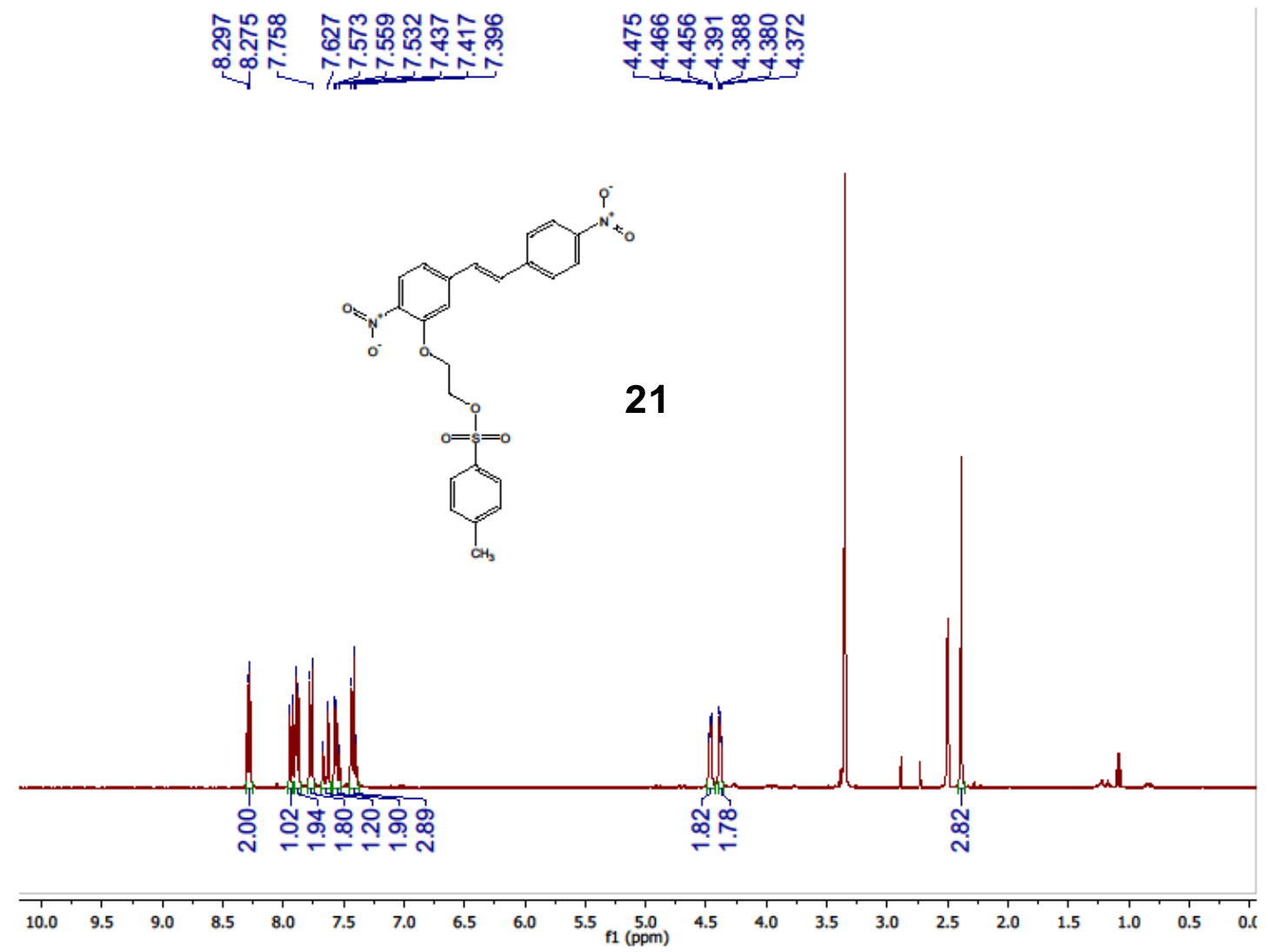




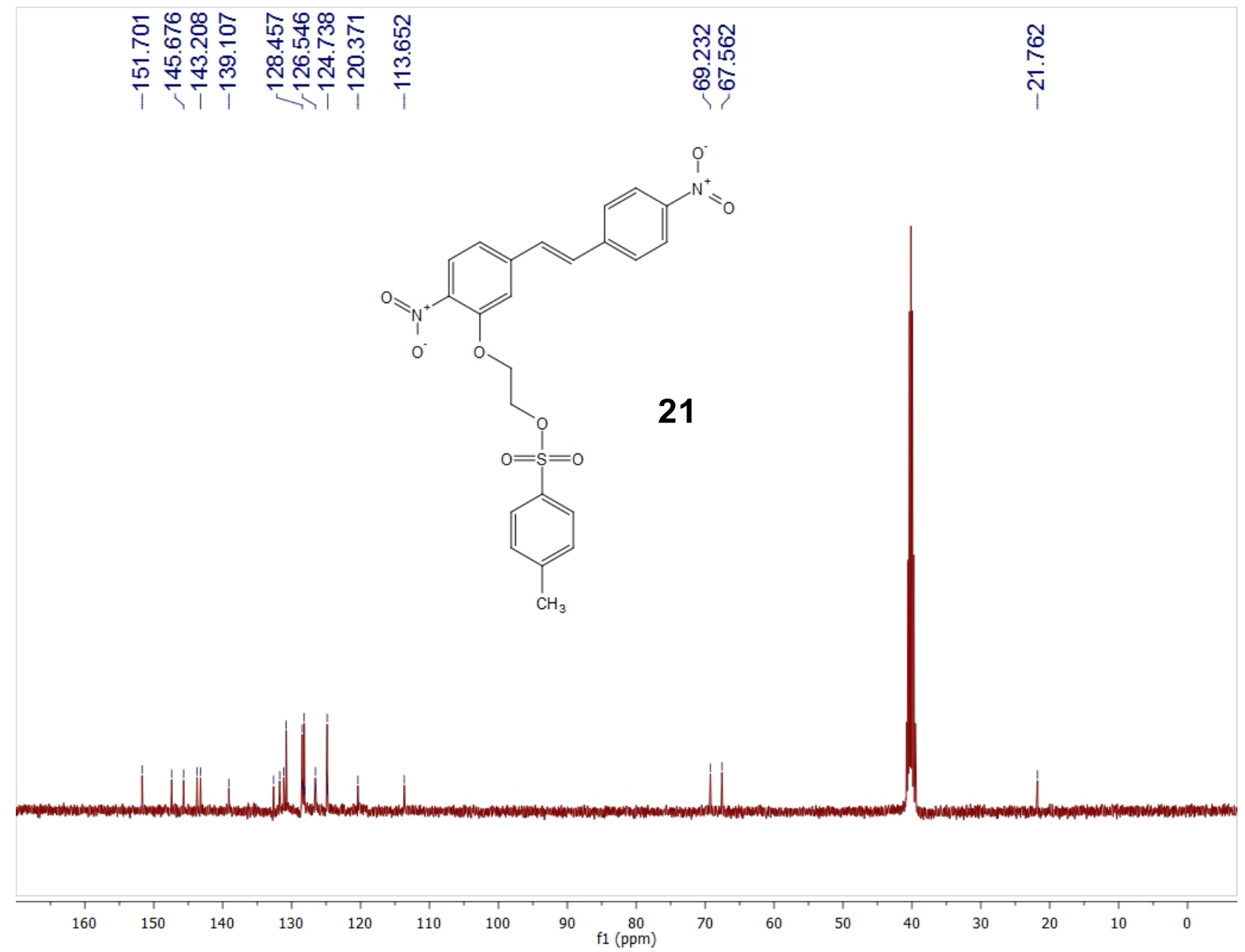




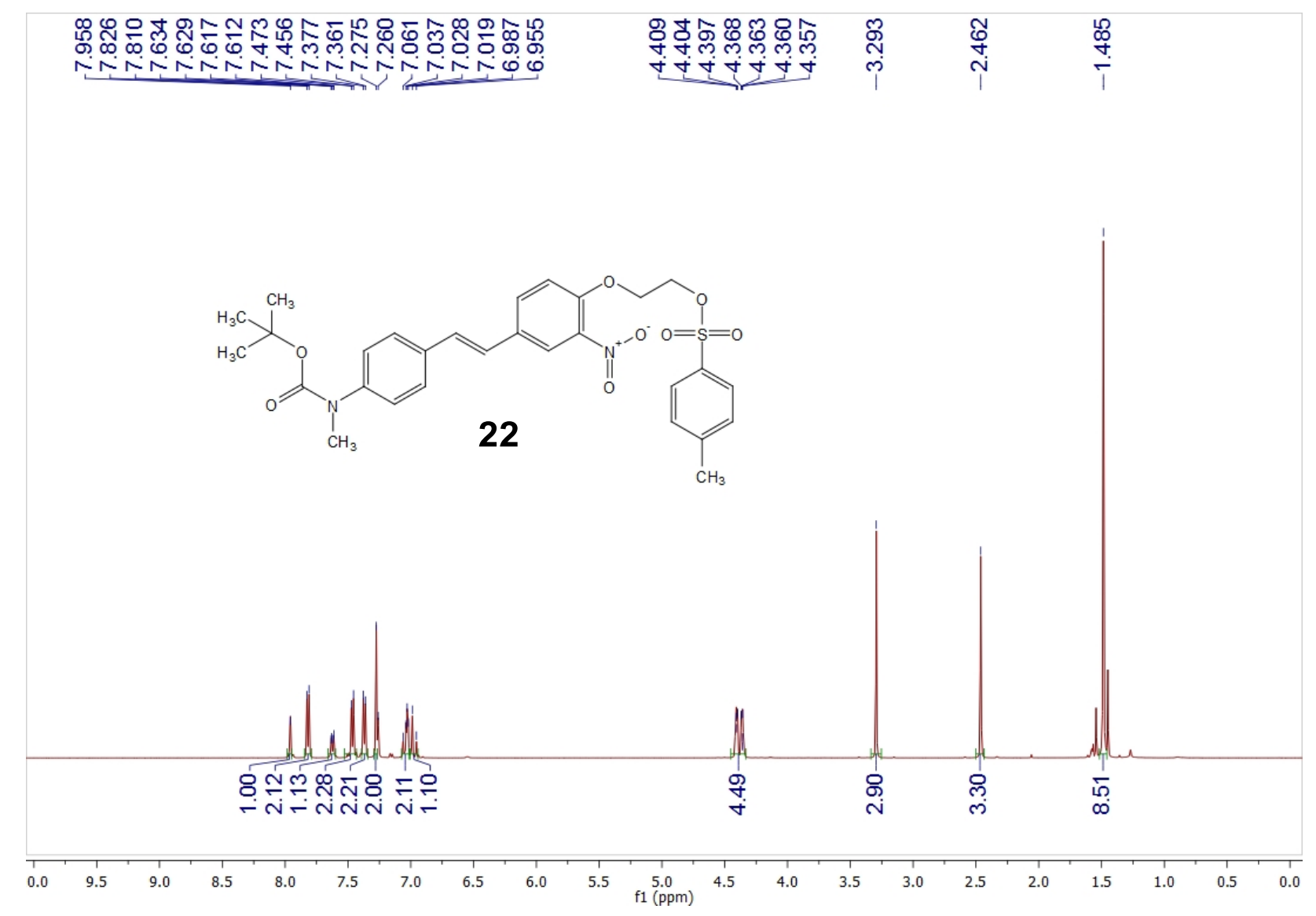




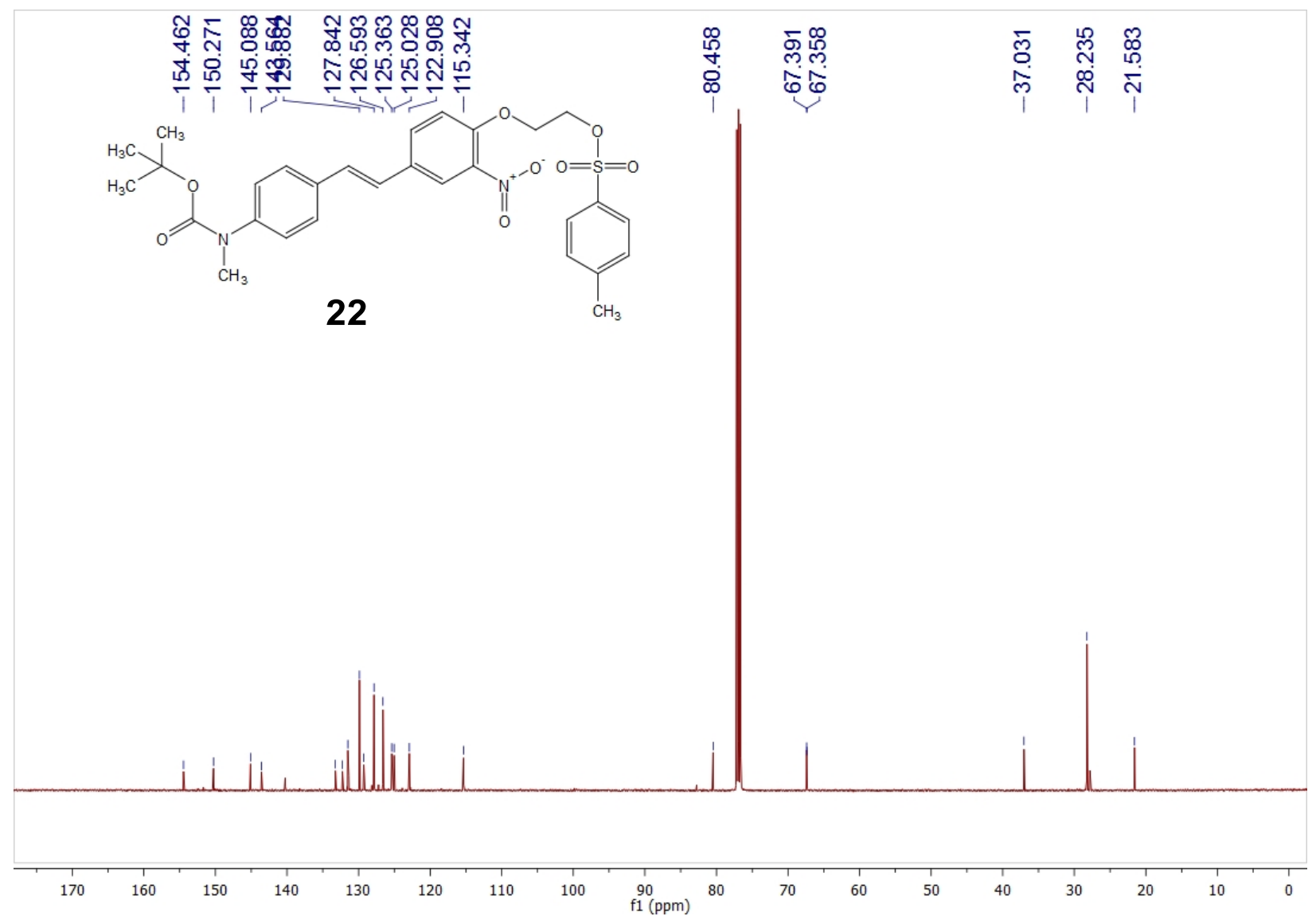



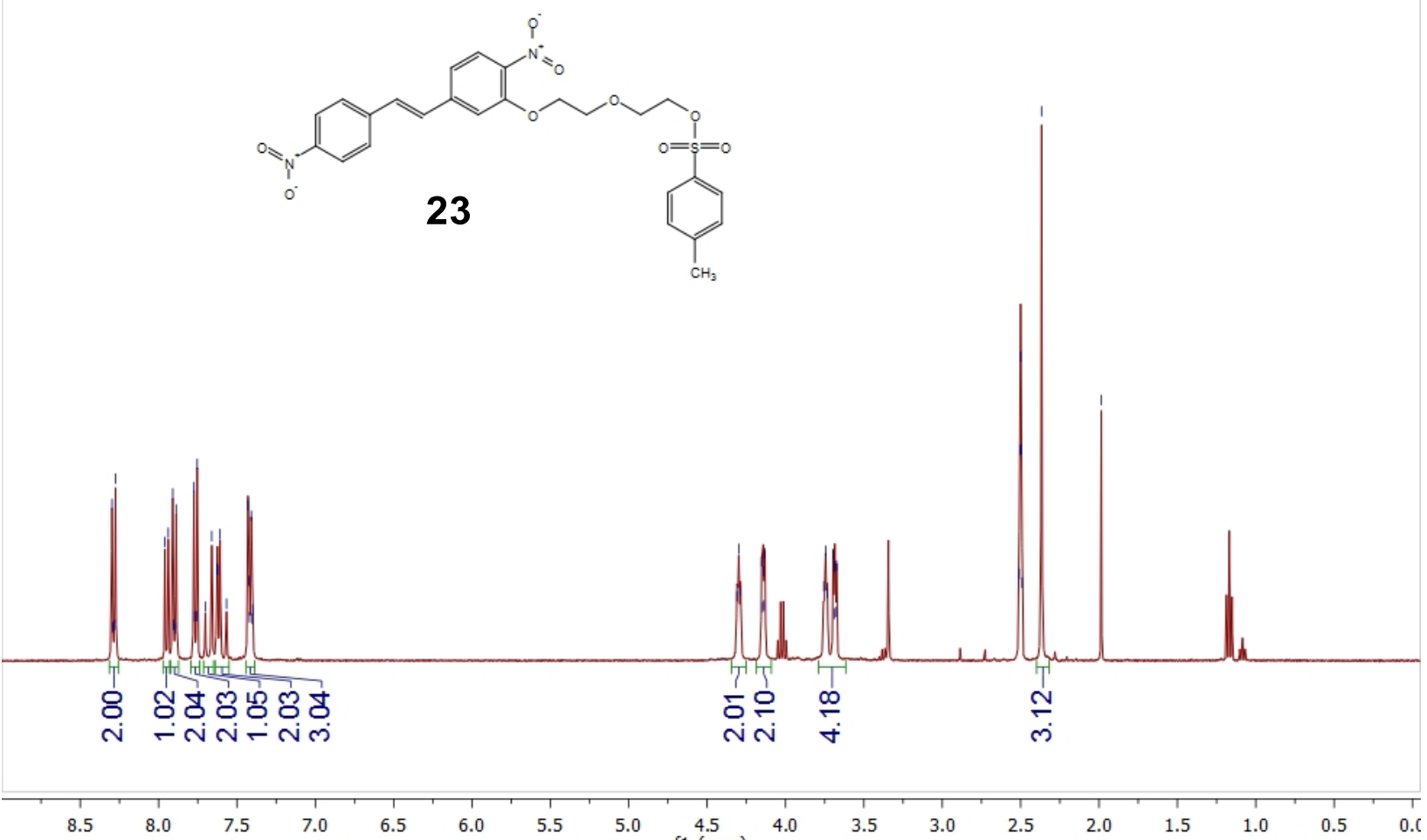


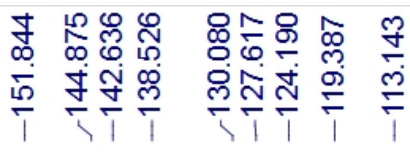

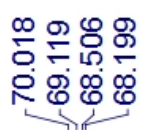

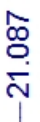
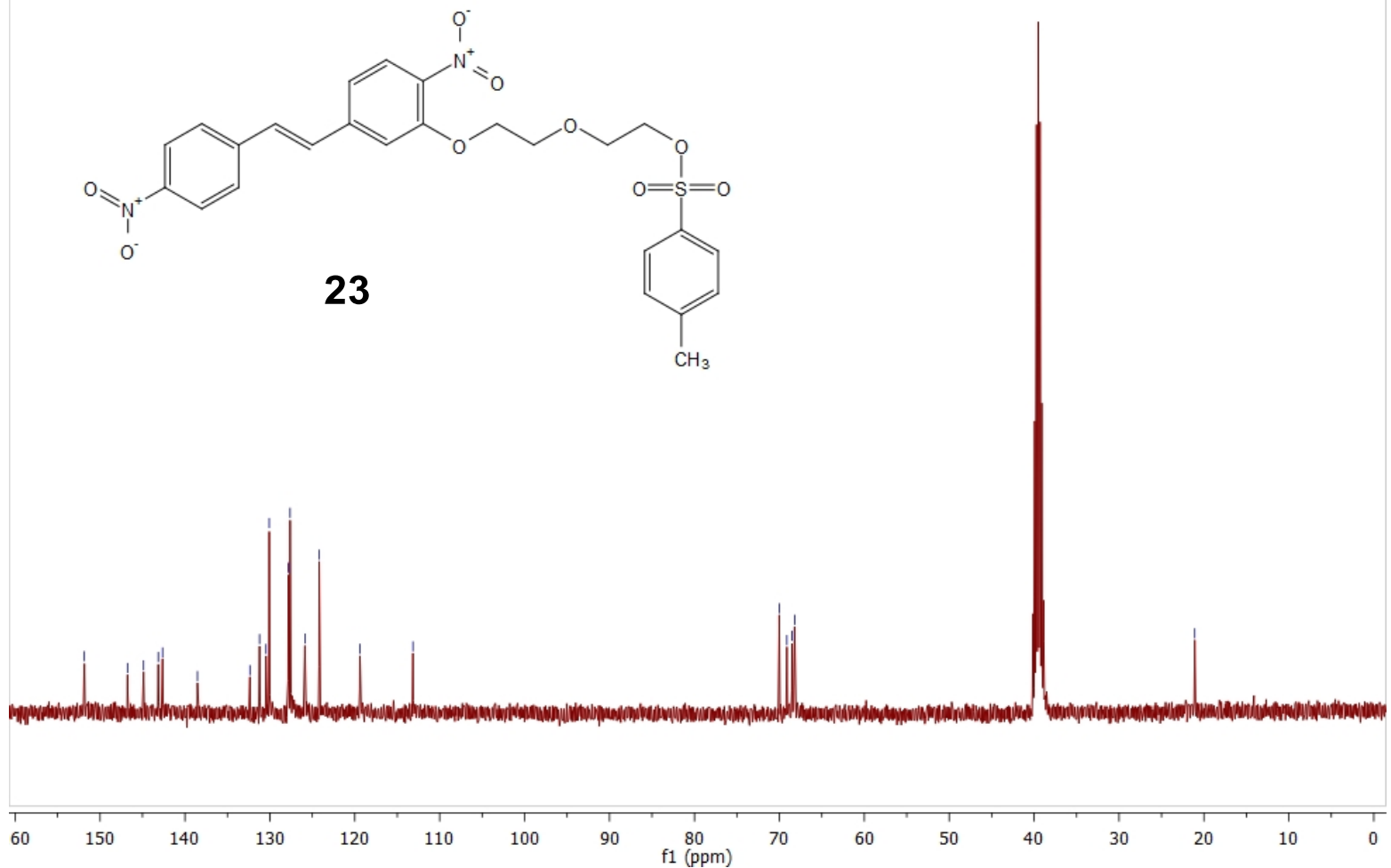


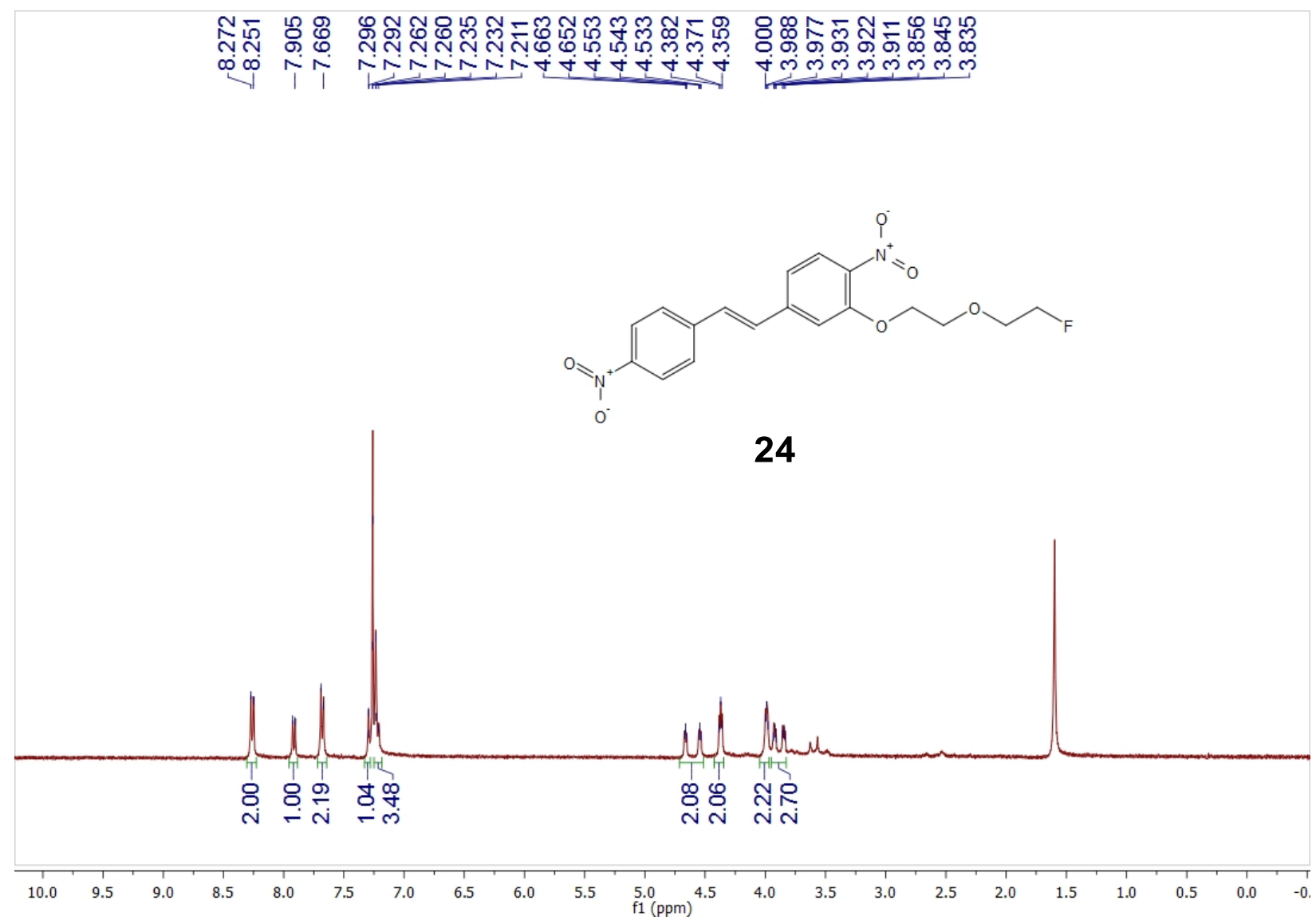




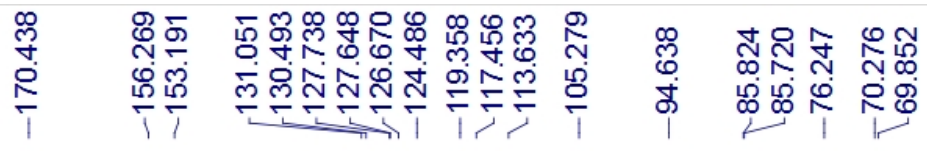

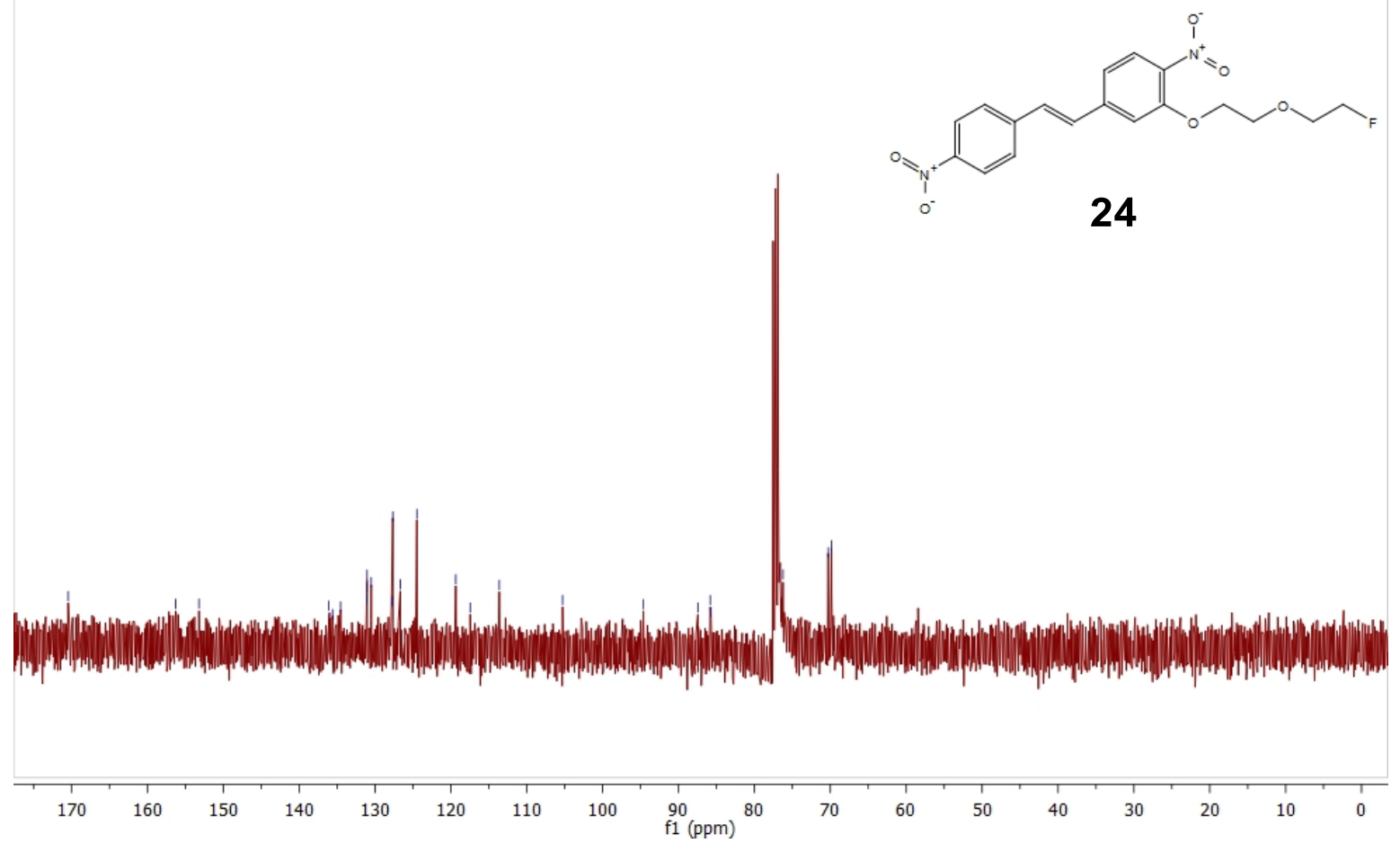




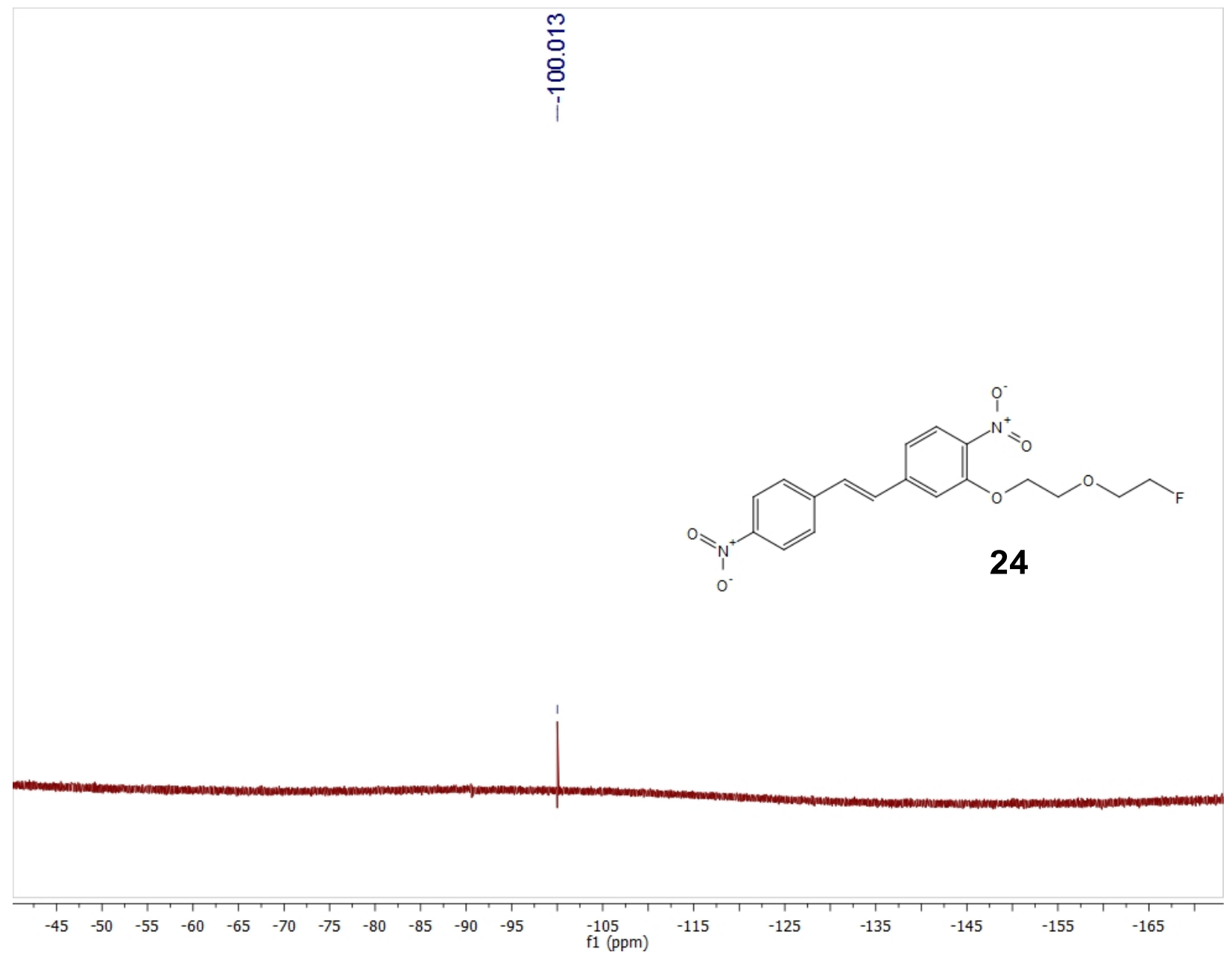




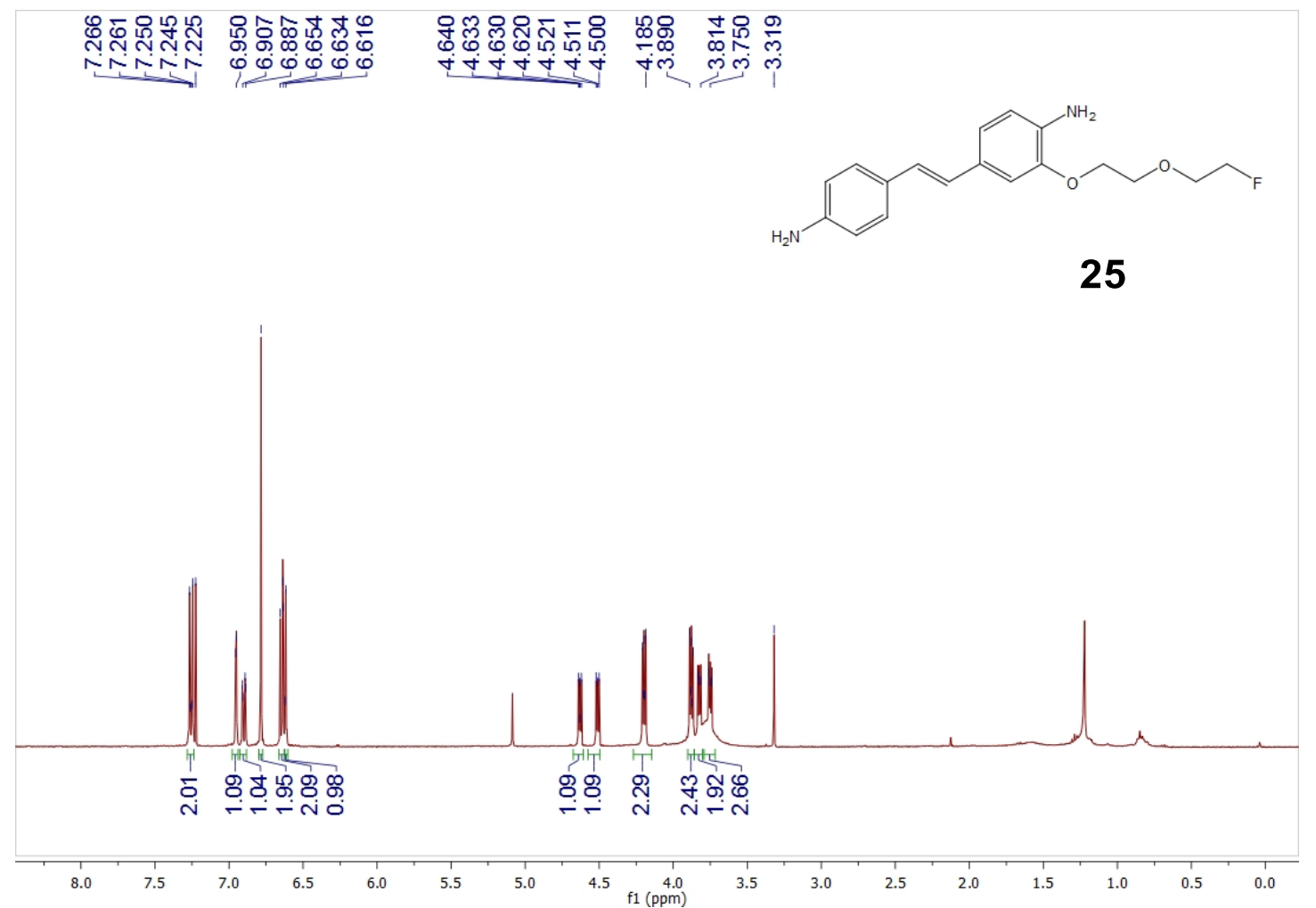




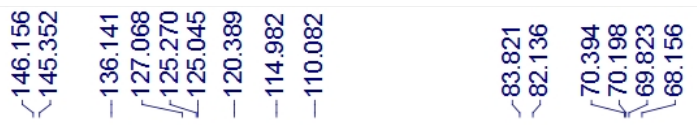
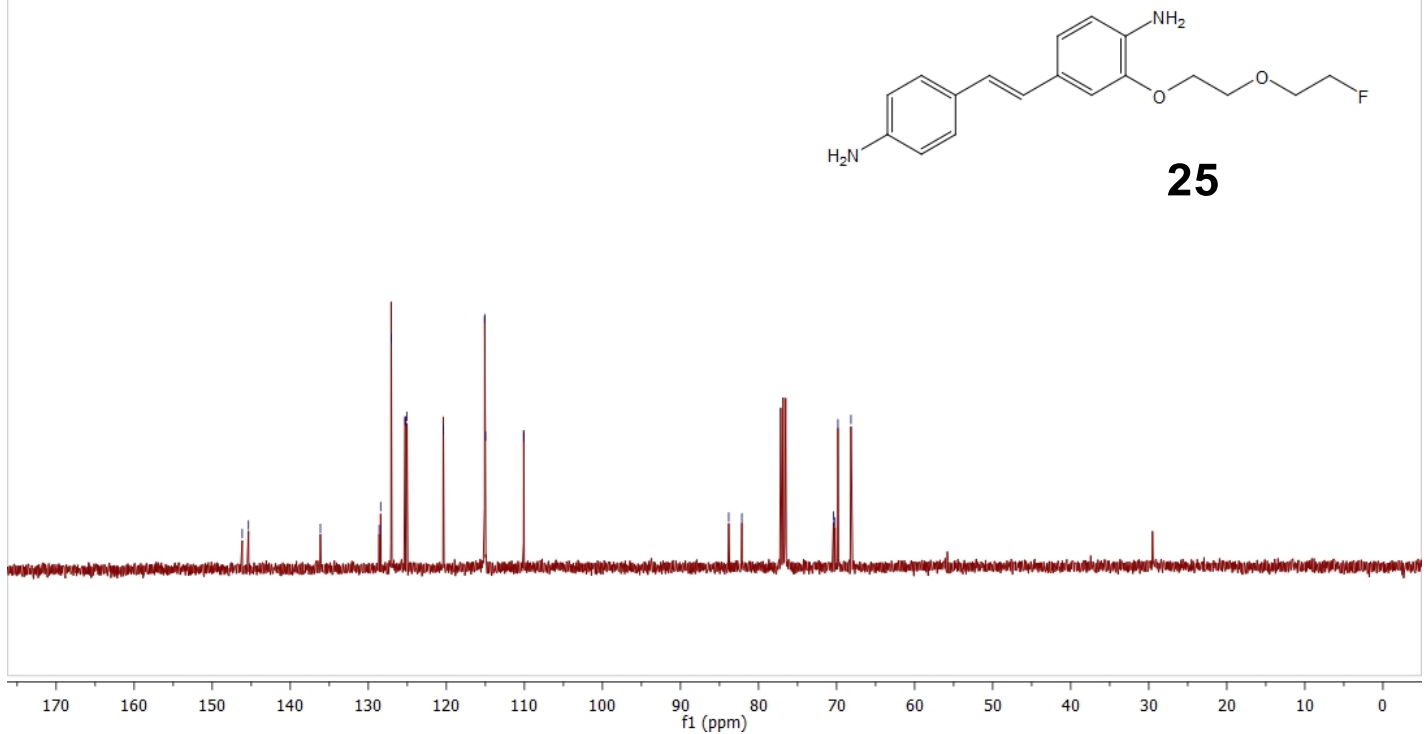


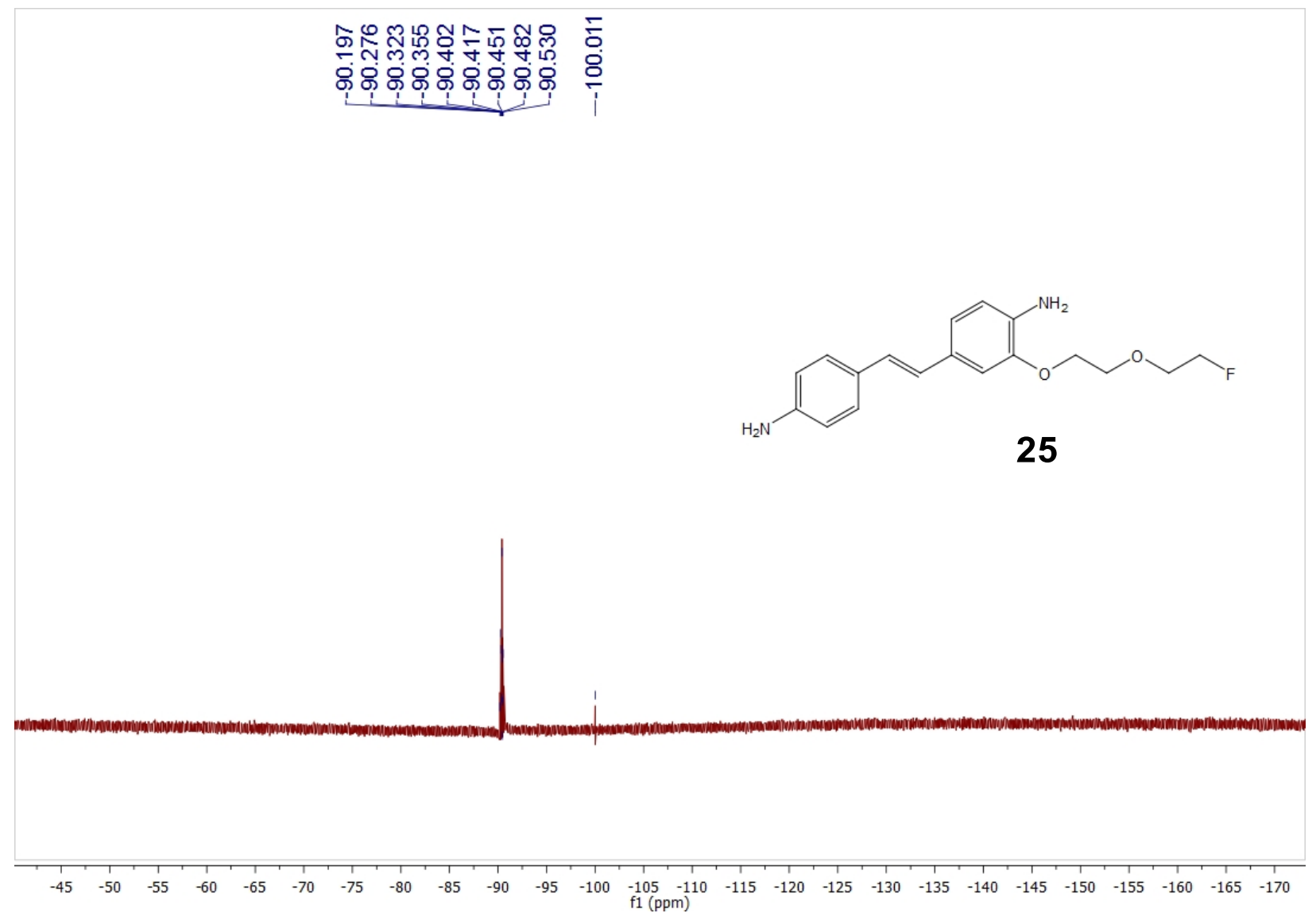




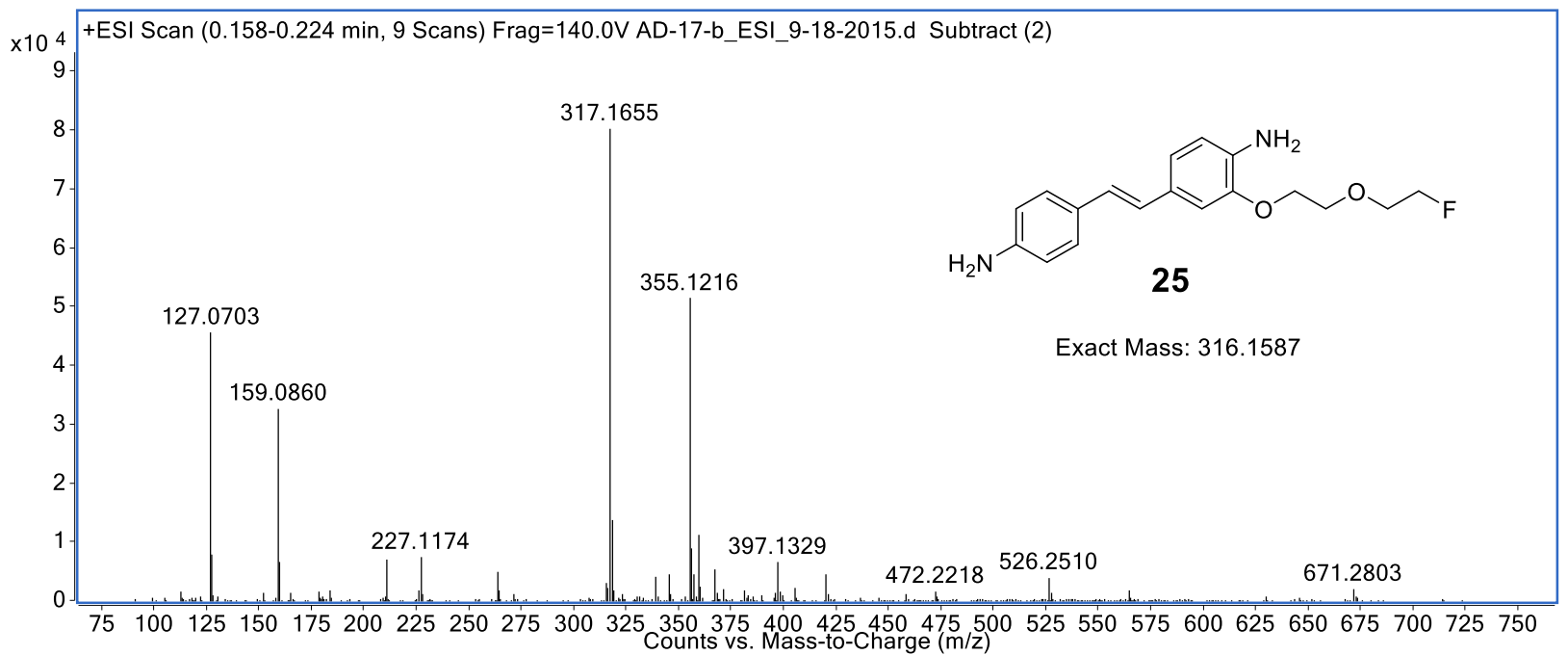



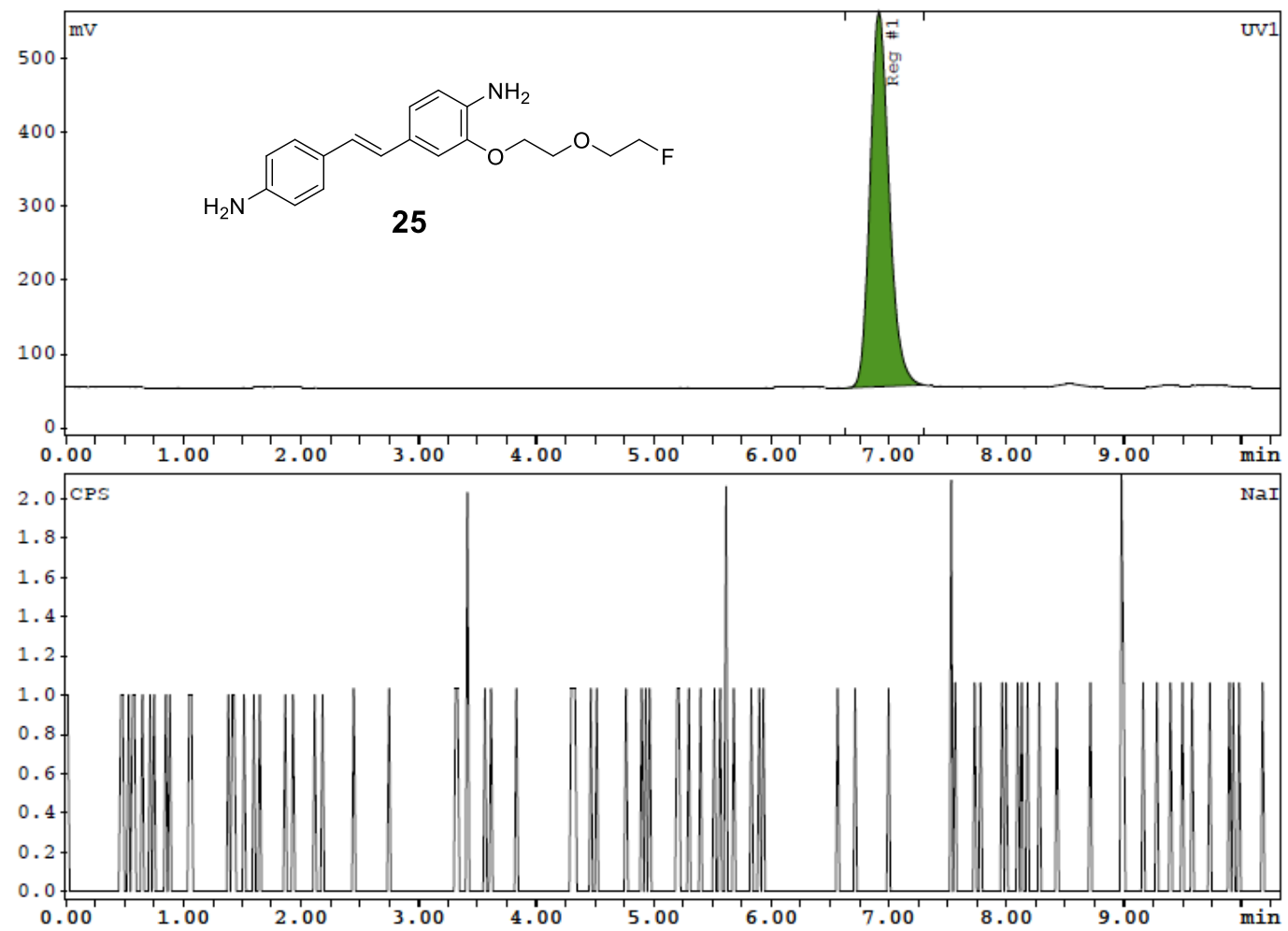

Sample description

\begin{tabular}{|c|c|c|}
\hline Measurement: & 10292015_105-2, injection : & 10/29/2015 10:28 AM \\
\hline Method: & ANAND COMPOUND from: & 10/29/2015 9:14 AM \\
\hline
\end{tabular}

Integration UV1
\begin{tabular}{|l|r|r|r|r|}
\hline Substance & $\mathrm{R} / \mathrm{T}$ & Type & Area & $\%$ Area \\
\hline & $\mathrm{min}$ & & $\mathrm{mV}^{\times} \mathrm{s}$ & $\%$ \\
\hline Reg \#1 & 6.92 & BB & 5662.917 & 100.00 \\
& & & & \\
\hline Total & & & 5662.917 & \\
\hline
\end{tabular}




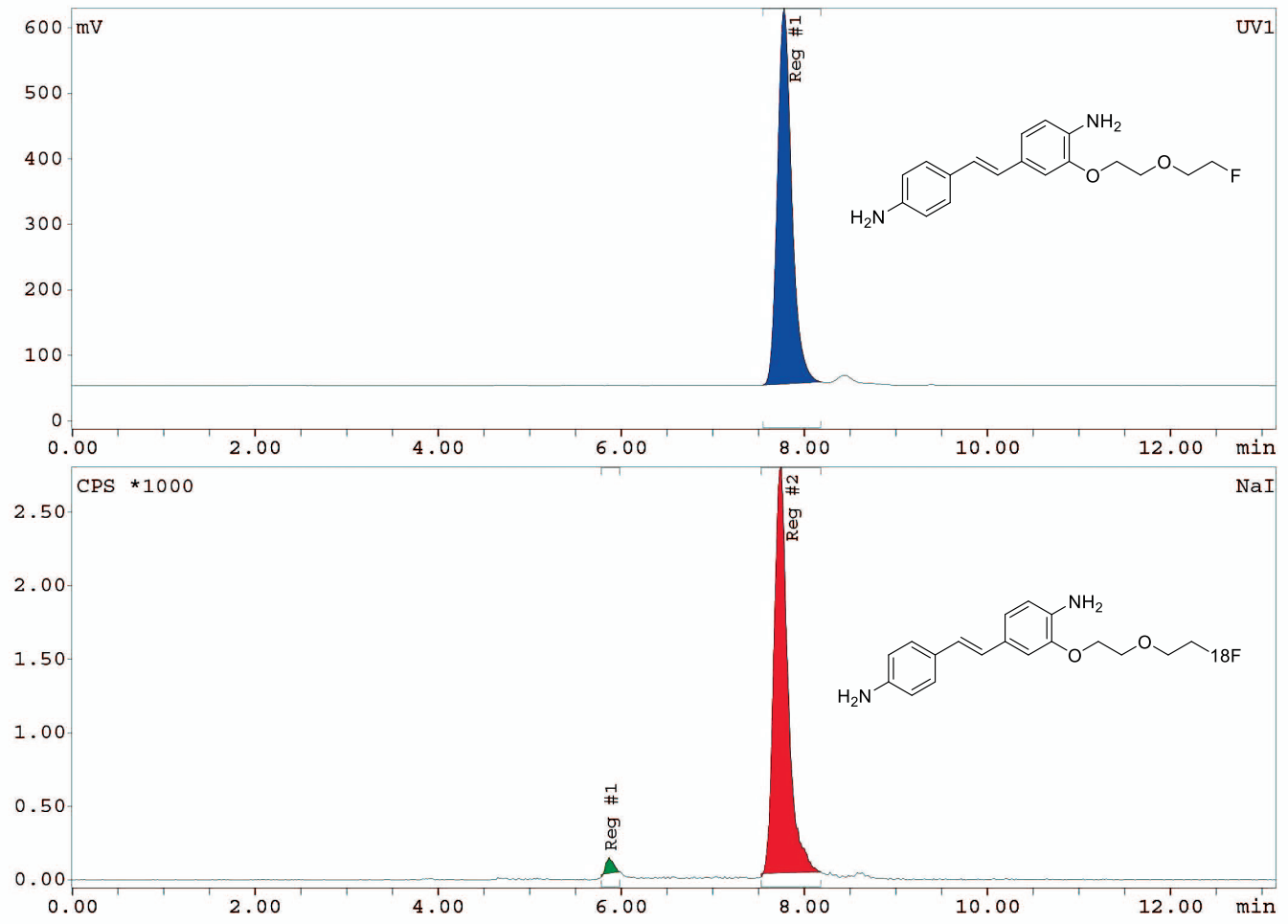

Sample description

$\begin{array}{lll}\text { Measurement: } & \text { 07162015_3, injection : } & \text { 7/16/2015 3:39 PM } \\ \text { Method: } & \text { ANAND COMPOUND from: } & \text { 7/16/2015 2:31 PM } \\ \text { Station number: } & 3 & \end{array}$

Timetable

\begin{tabular}{|c|c|c|c|c|c|}
\hline No. & Time, $\mathbf{m}$ & Event type & Value & Unit & Comment \\
\hline 1 & 1.15 & Gradient & Hold & & \\
\hline 2 & 1.18 & Gradient & Hold & & \\
\hline 3 & 13.17 & Manual stop & Stp & & \\
\hline
\end{tabular}

\begin{tabular}{|l|r|r|r|r|}
\hline Integration Nal & \multicolumn{3}{|c|}{} \\
\hline Substance & R/T & Type & Area & $\%$ Area \\
\hline & min & & Counts & $\%$ \\
\hline Reg \#1 & 5.87 & BB & 550.29 & 1.85 \\
Reg \#2 & 7.75 & BB & 29188.72 & 98.15 \\
\hline Total & & & & \\
Total area & & & 29739.01 & \\
\hline & & & 35927.25 & \\
\hline Integration UV1 & & & & \\
\hline Substance & R/T & Type & Area & $\%$ Area \\
\hline & min & & mV*s & $\%$ \\
\hline Reg \#1 & 7.78 & BB & 6196.199 & 100.00 \\
\hline Total & & & & \\
\hline
\end{tabular}




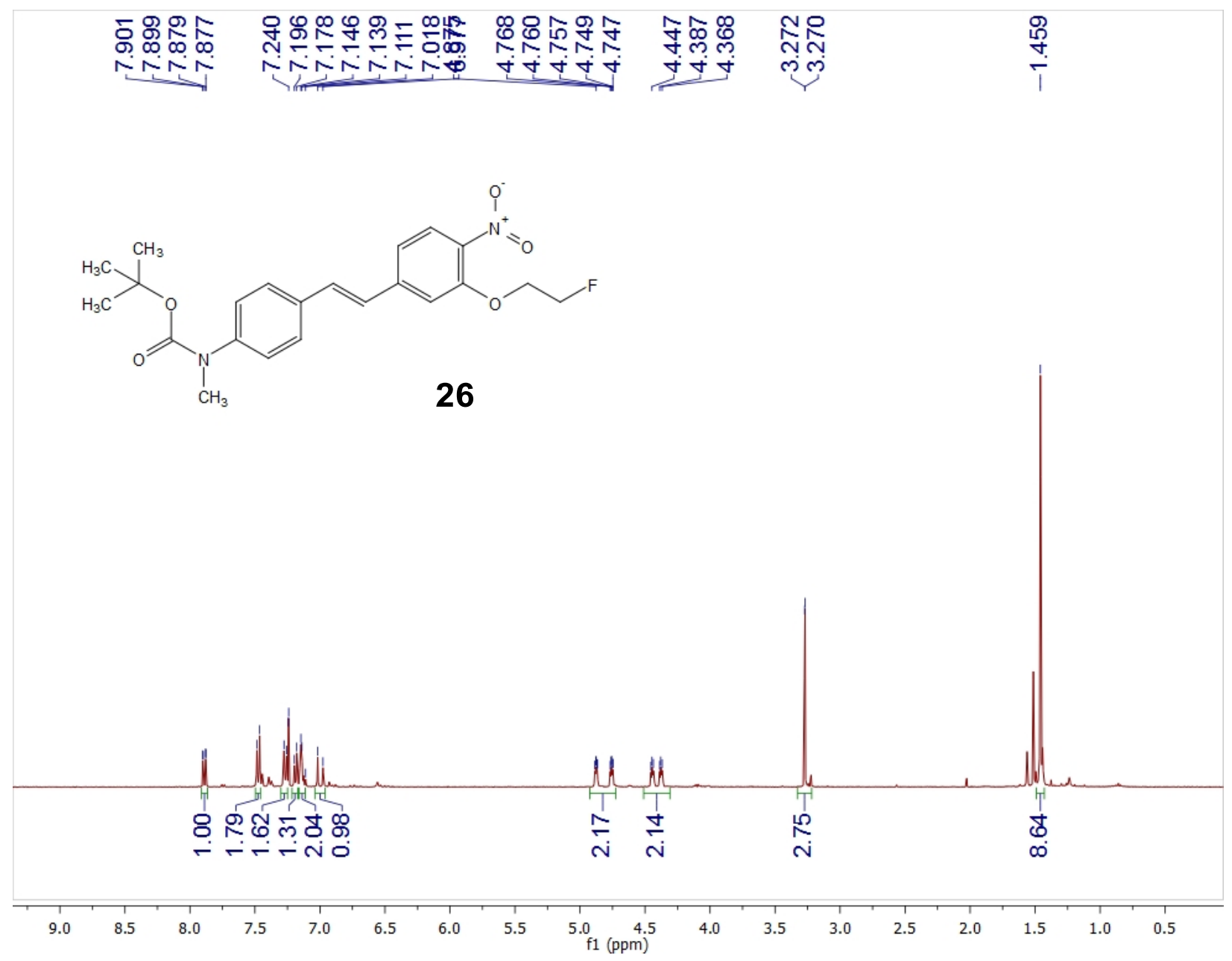




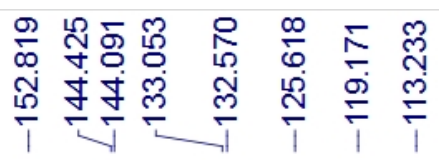

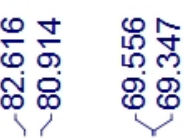

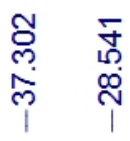
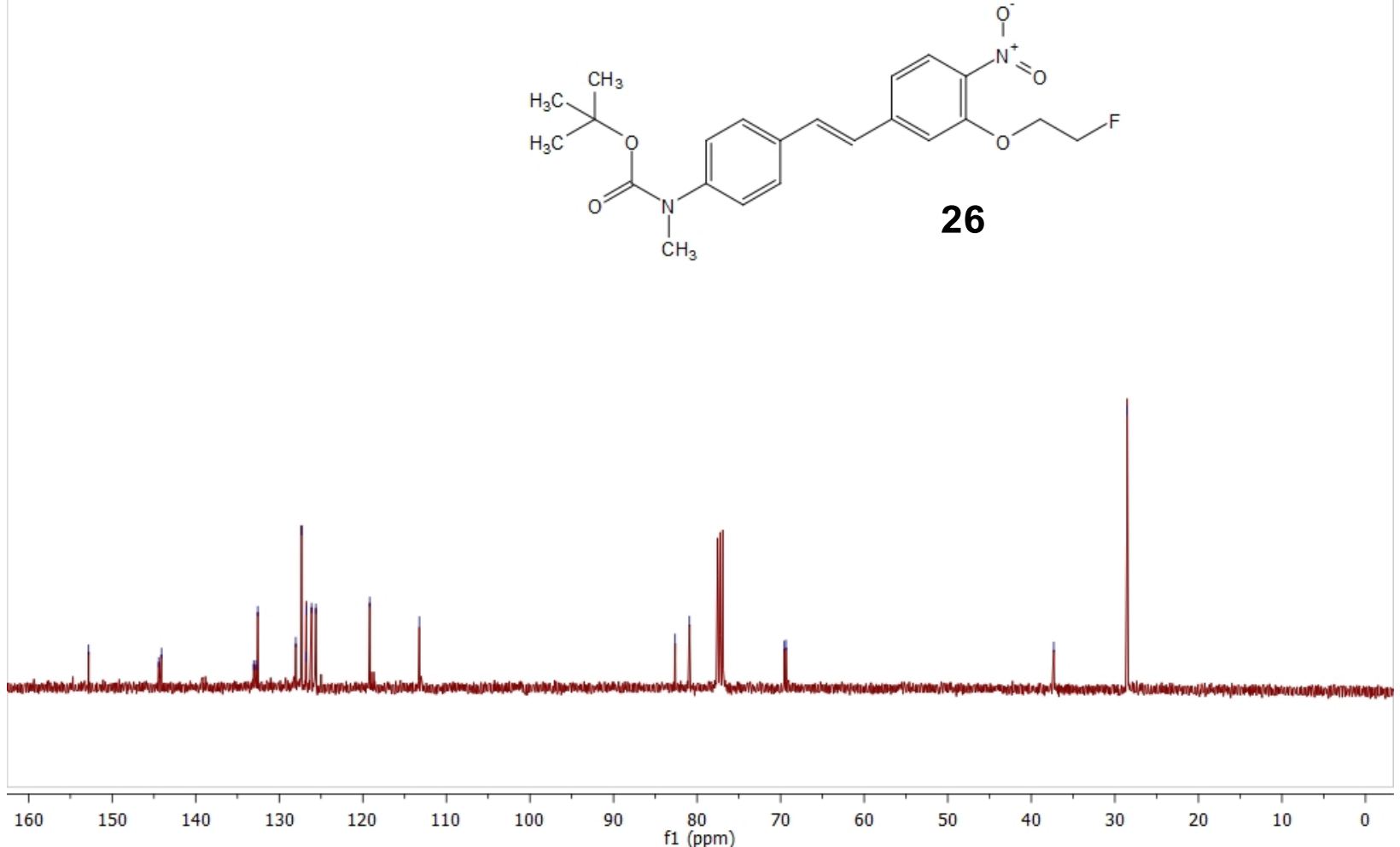


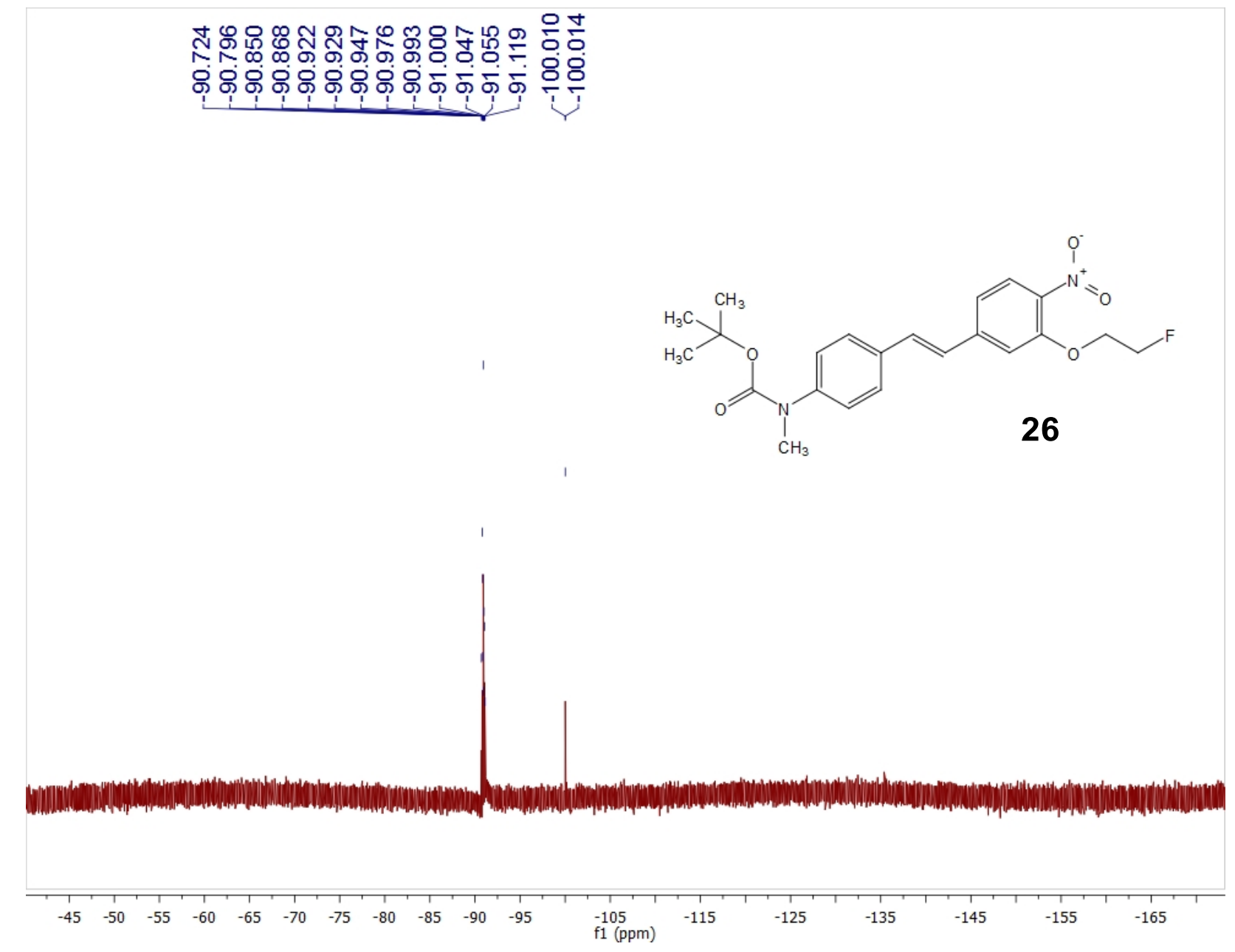




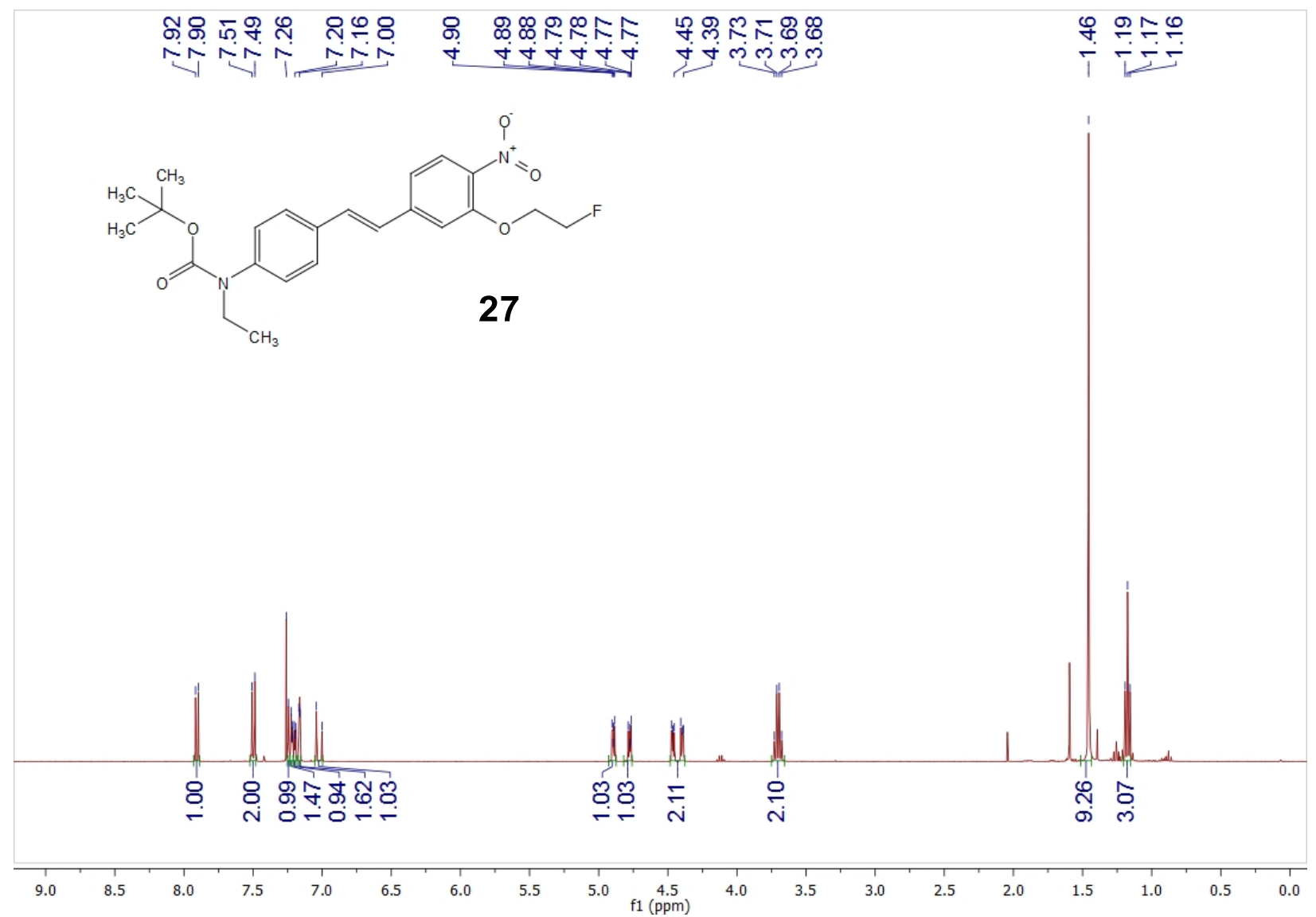




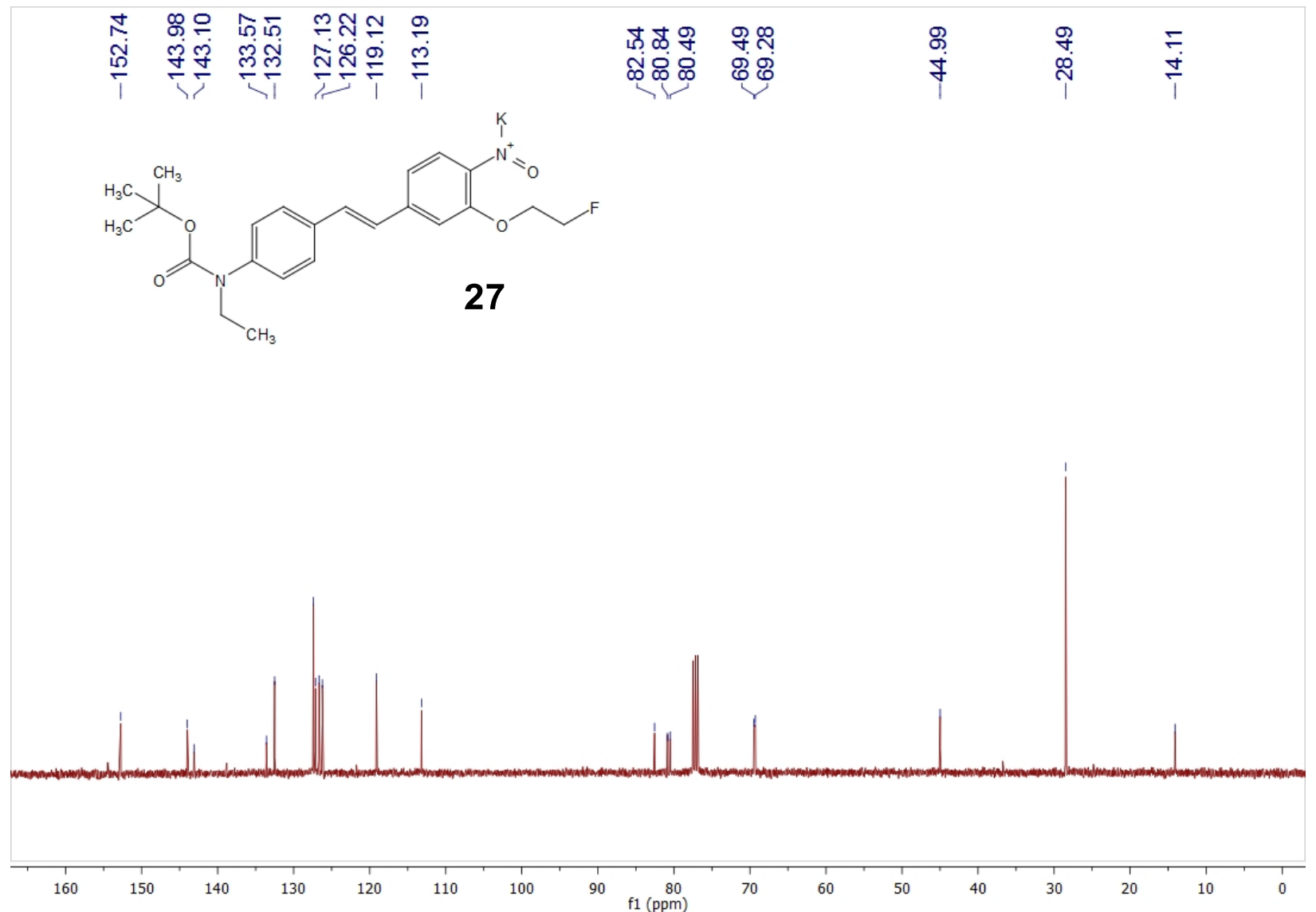




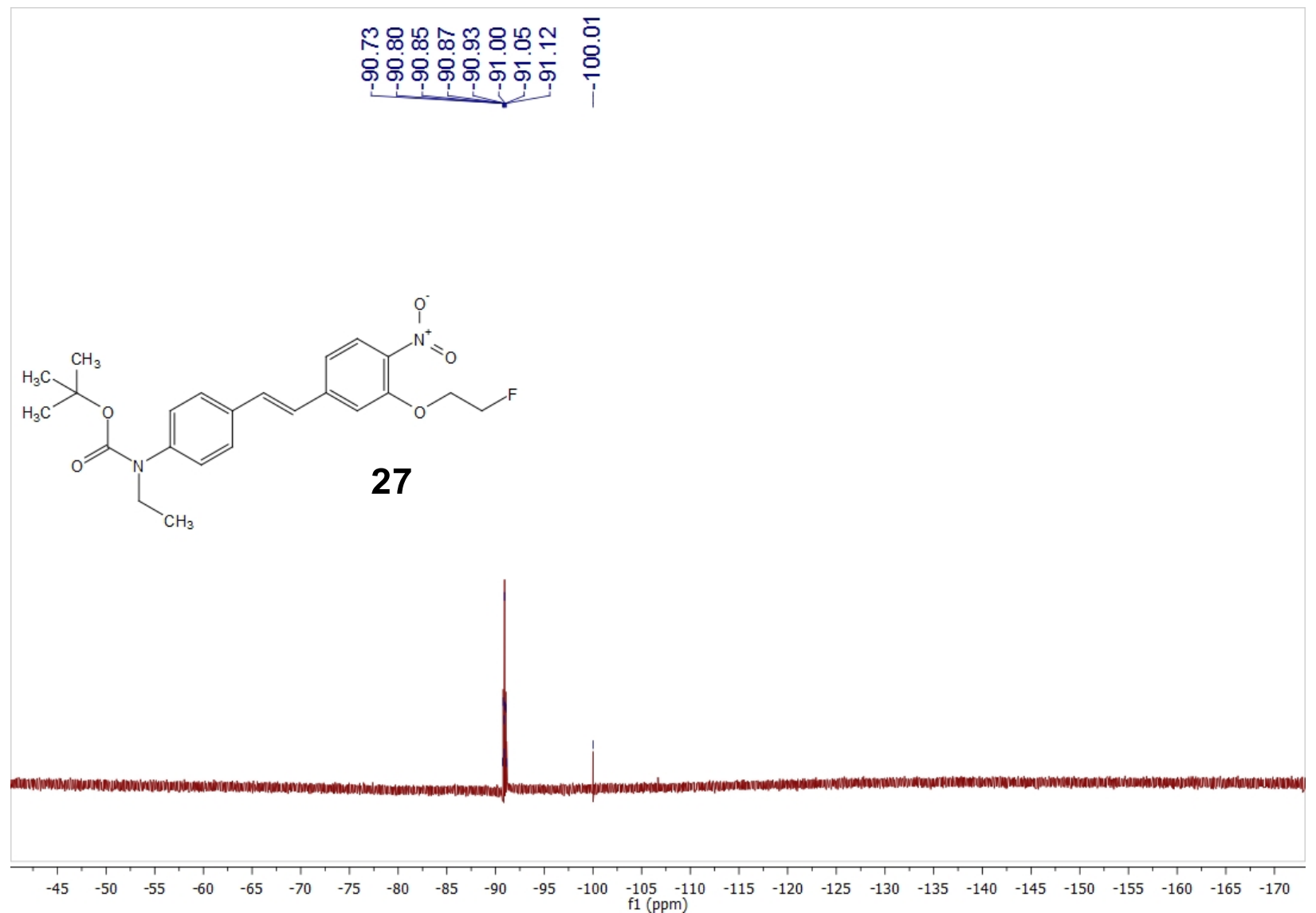




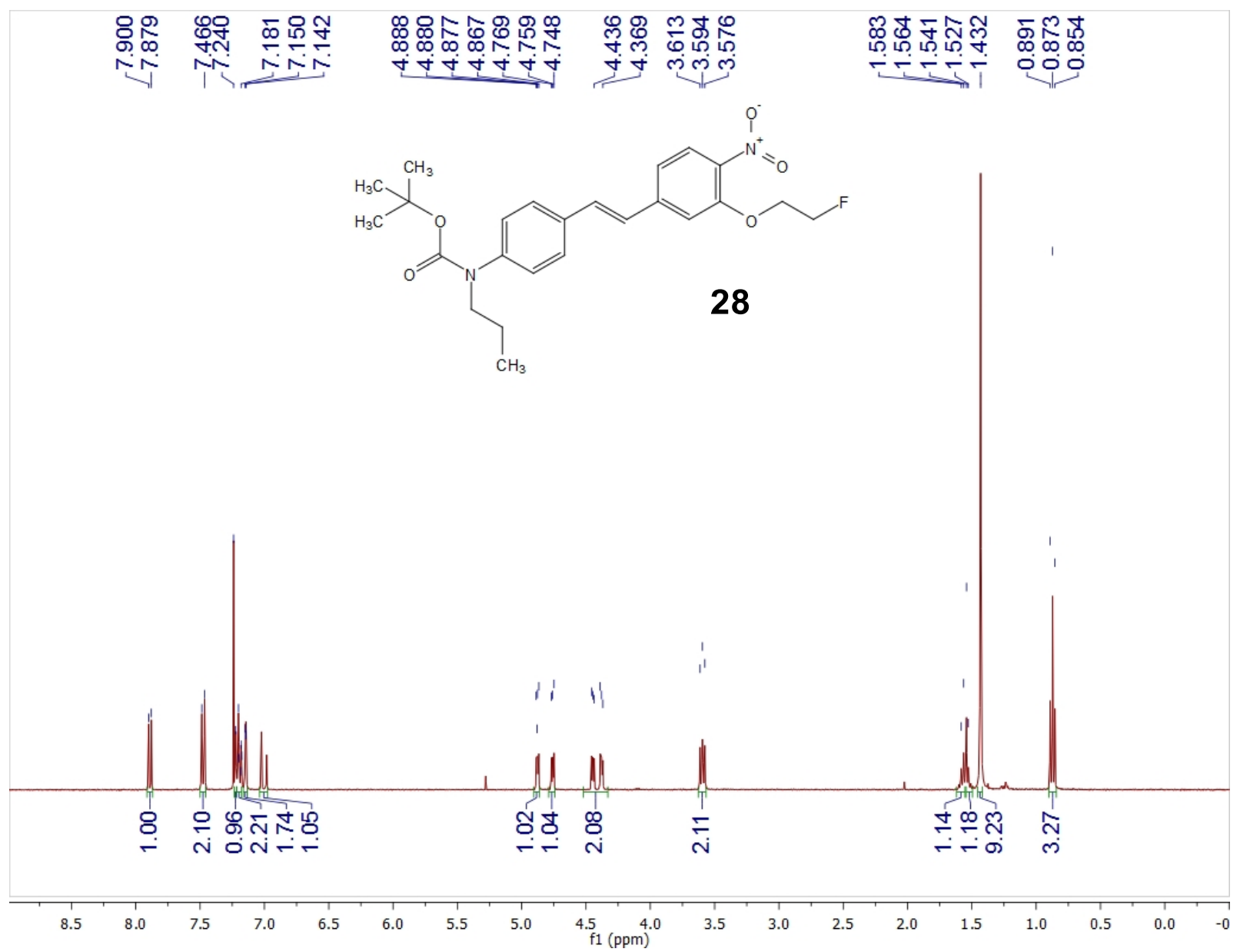




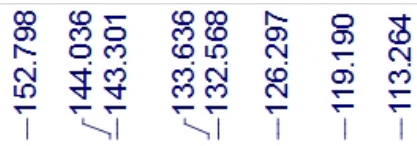

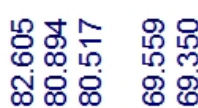

$\stackrel{\substack{8 \\ \text { in }}}{1}$

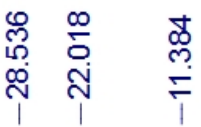
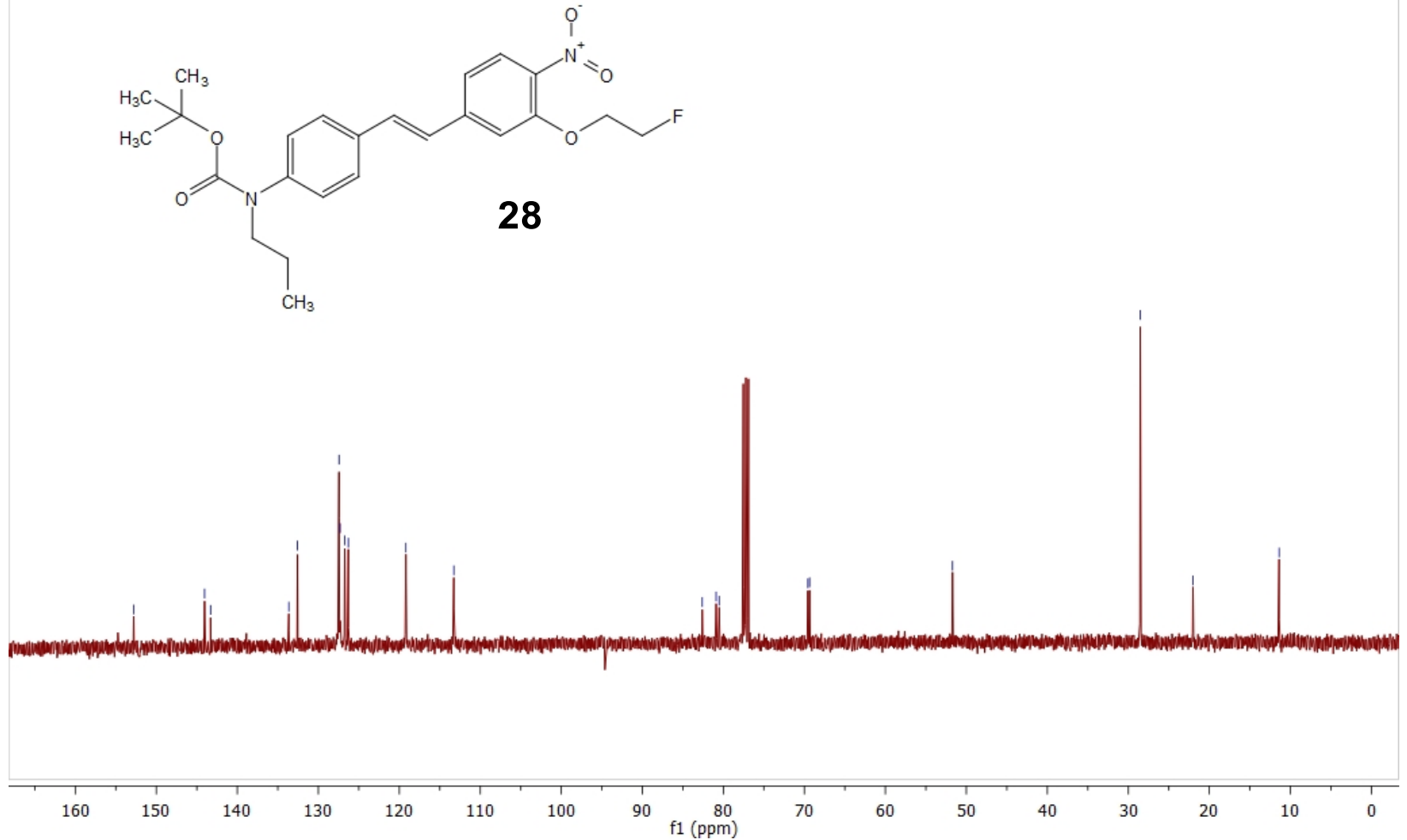


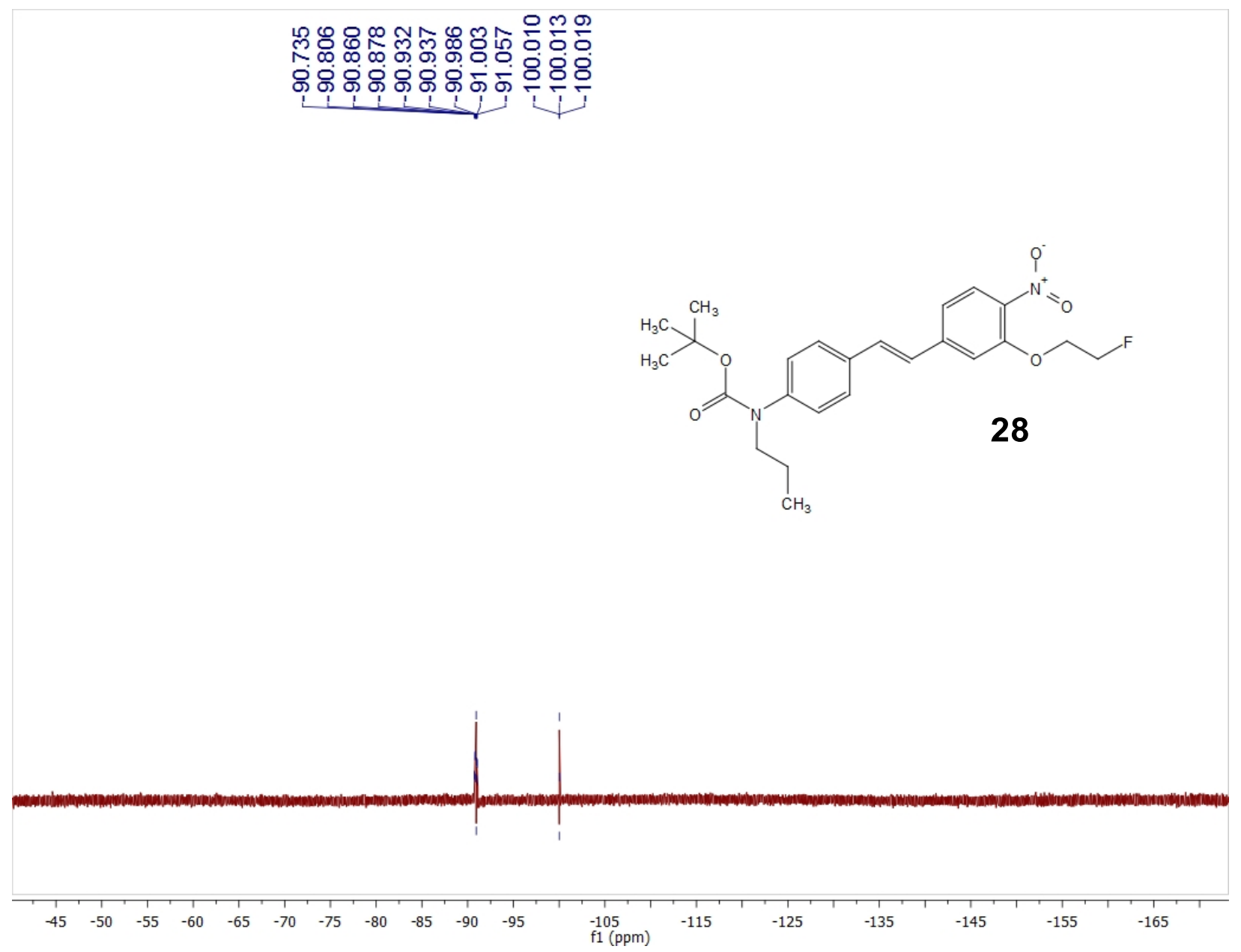




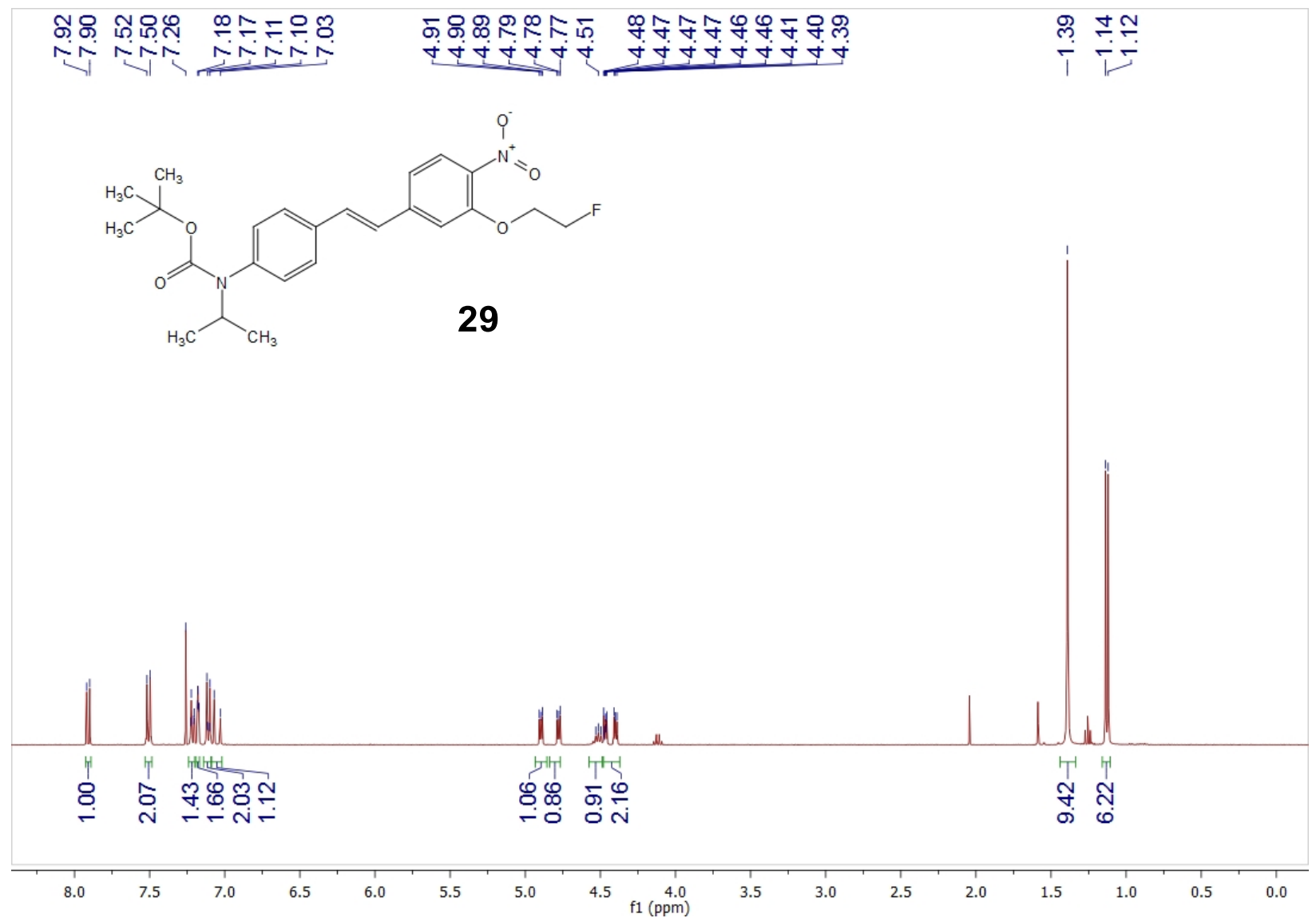




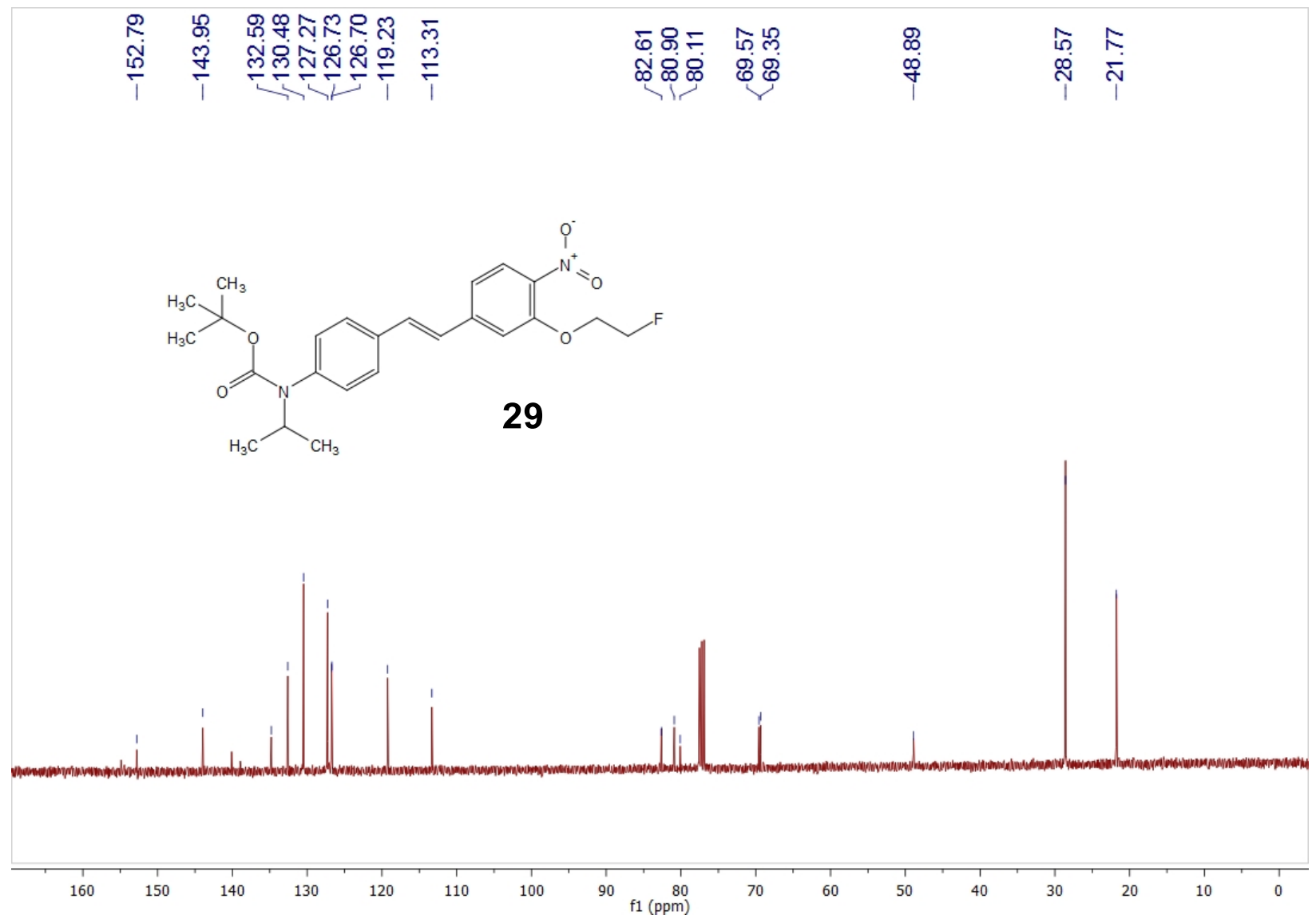




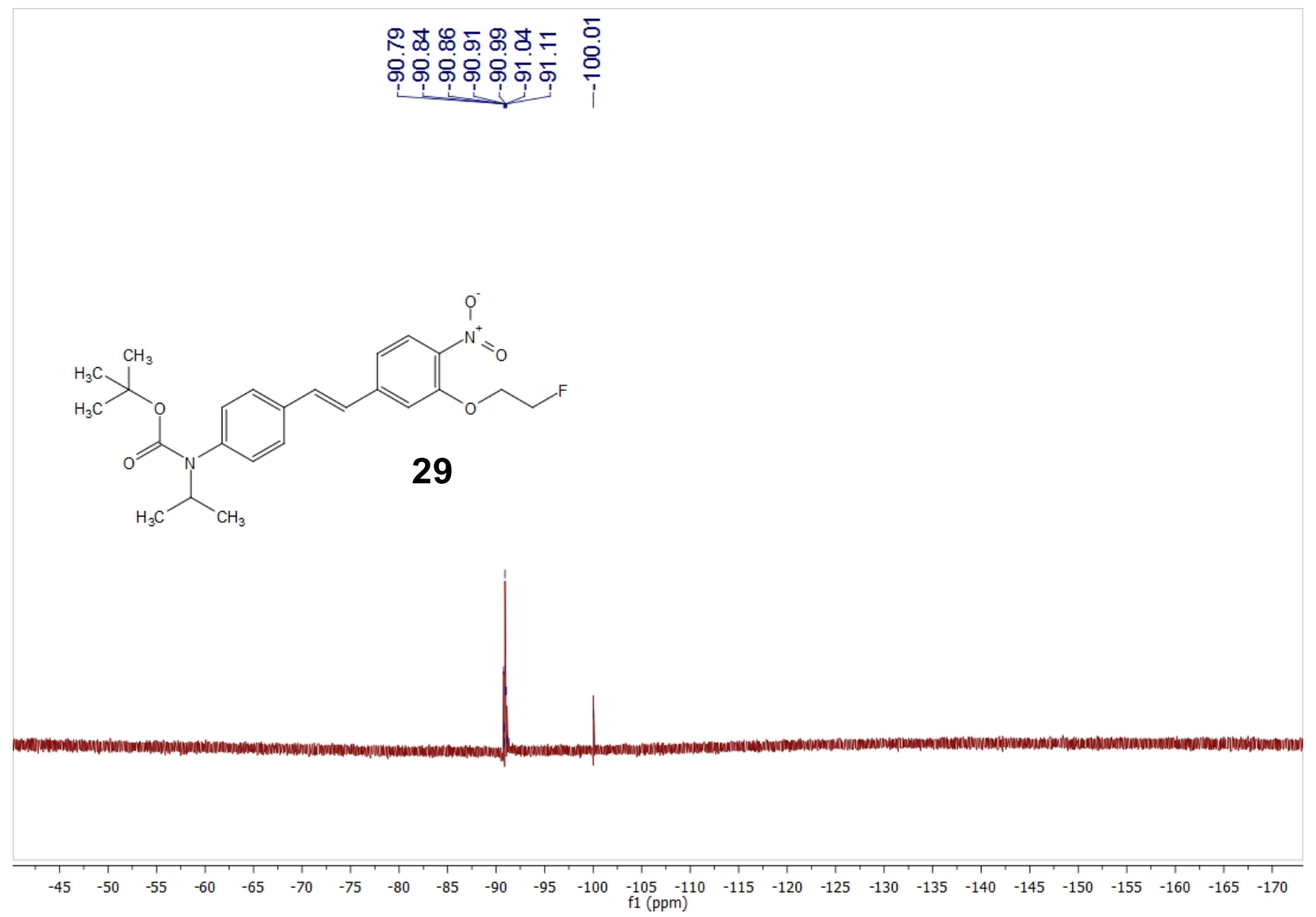




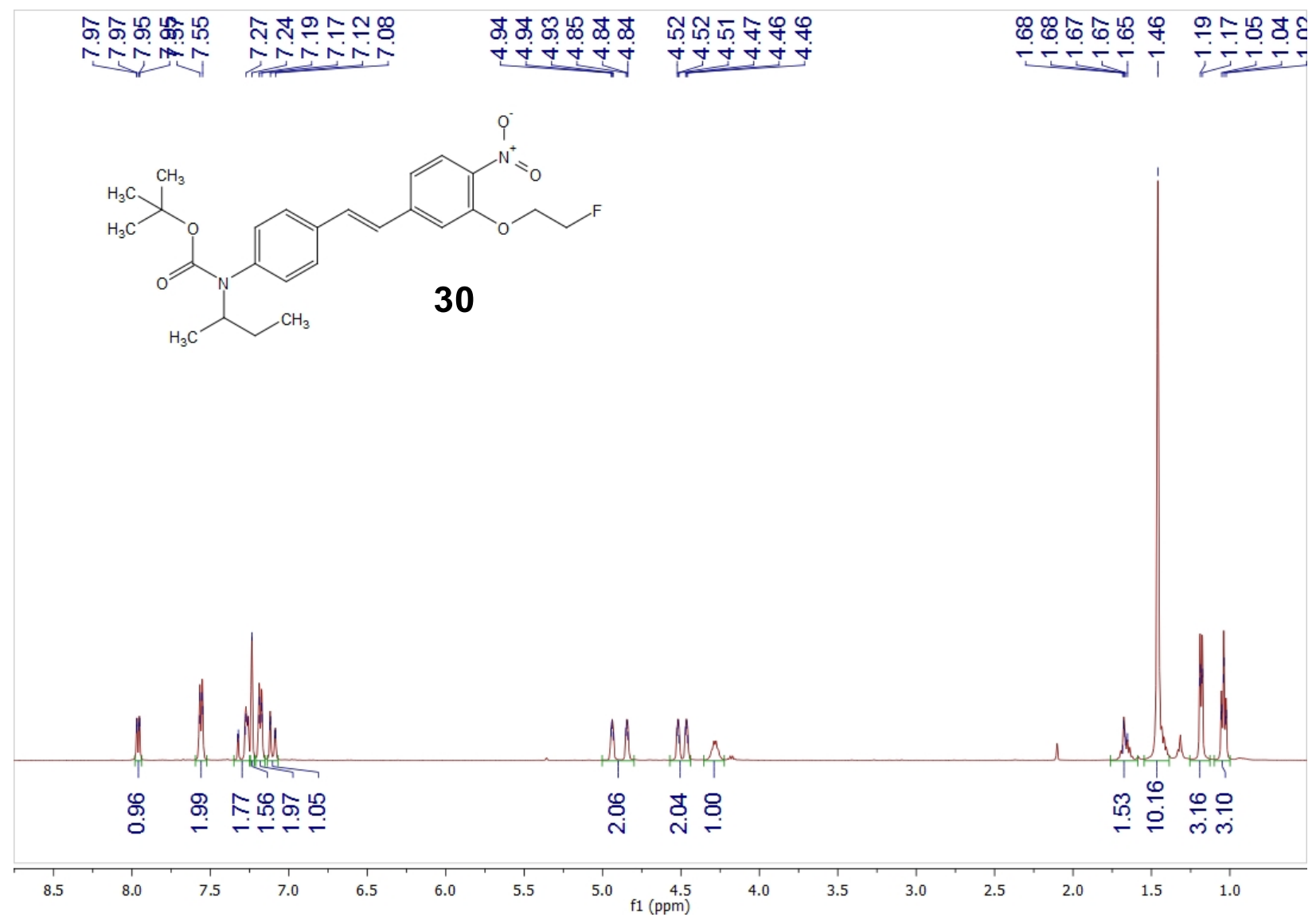




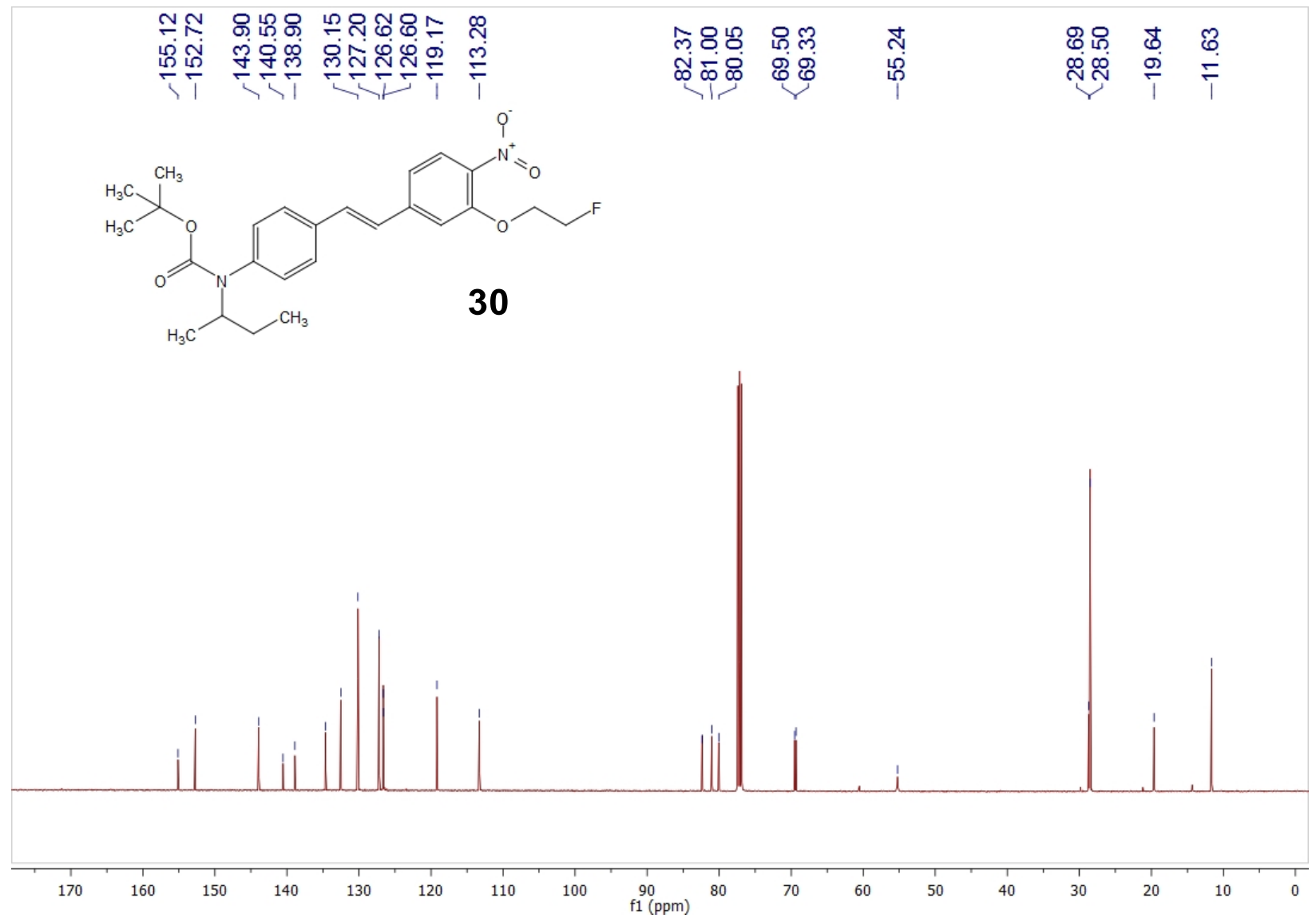




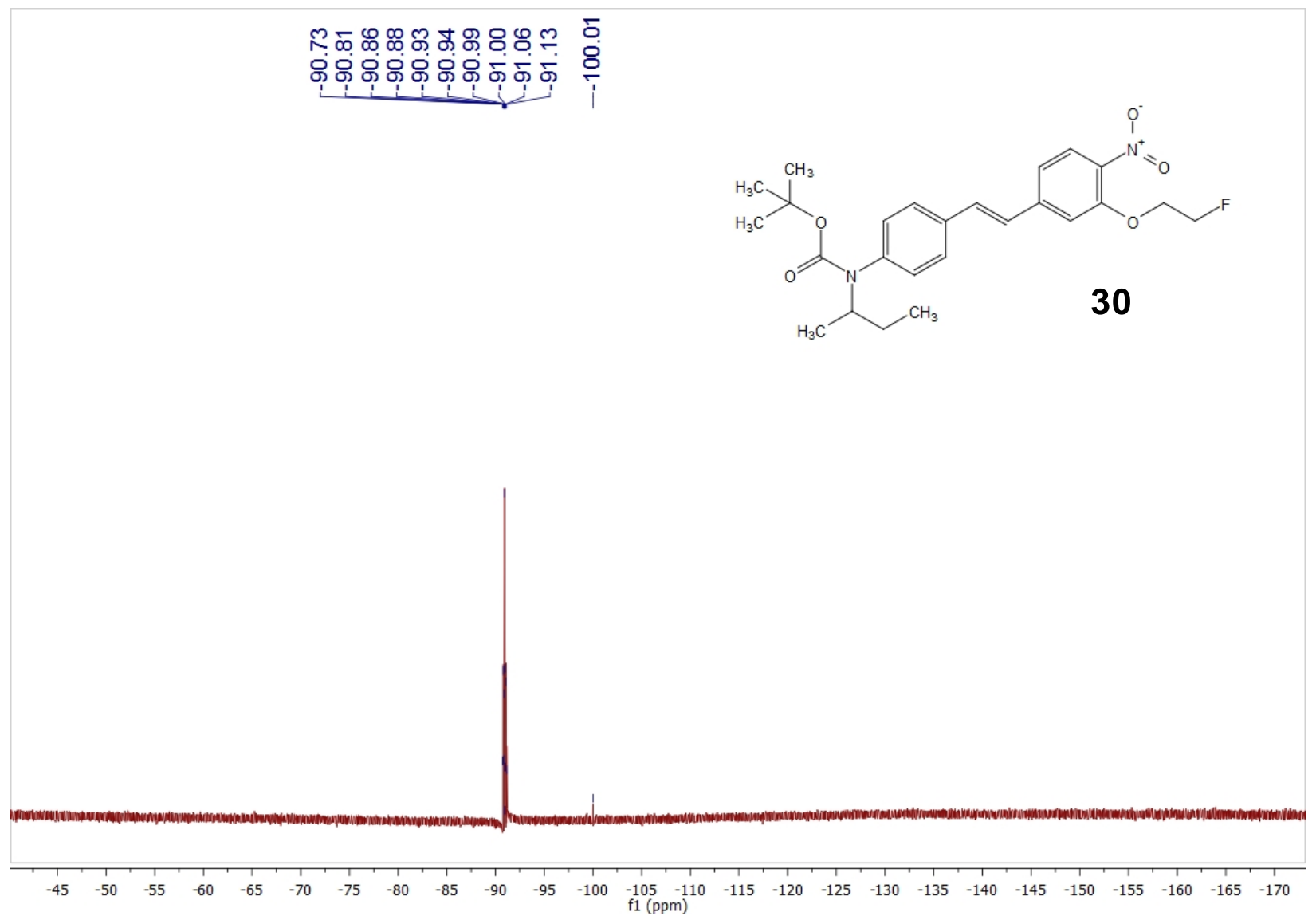




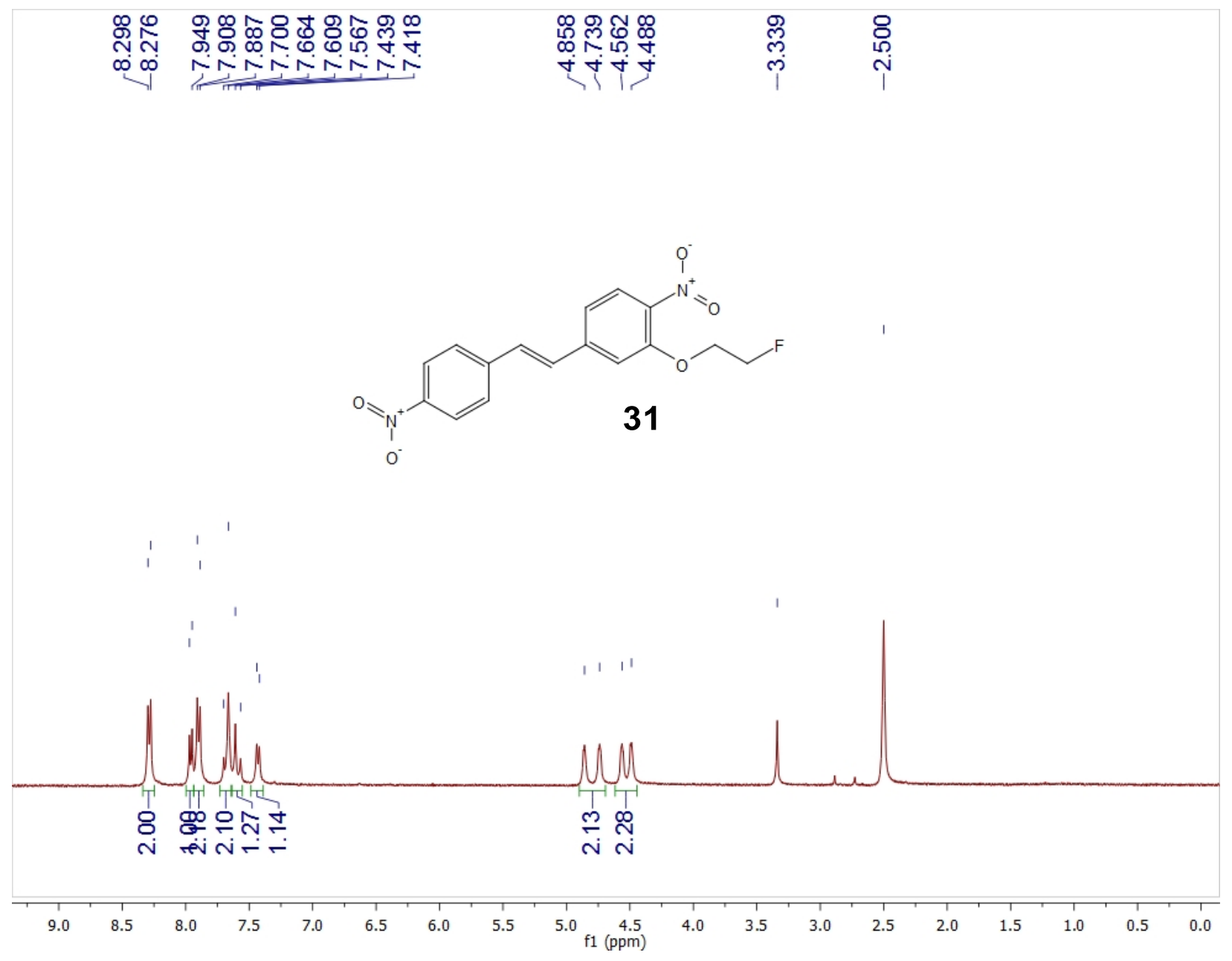




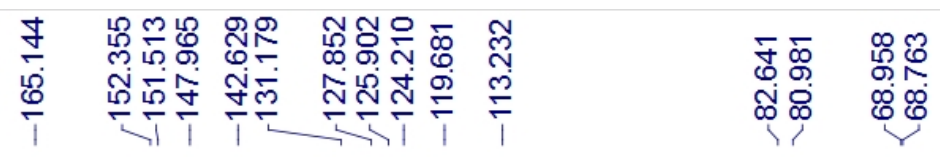<smiles>O=[N+]([O-])c1ccc(/C=C/c2ccc([N+](=O)[O-])c(OCCF)c2)cc1</smiles>

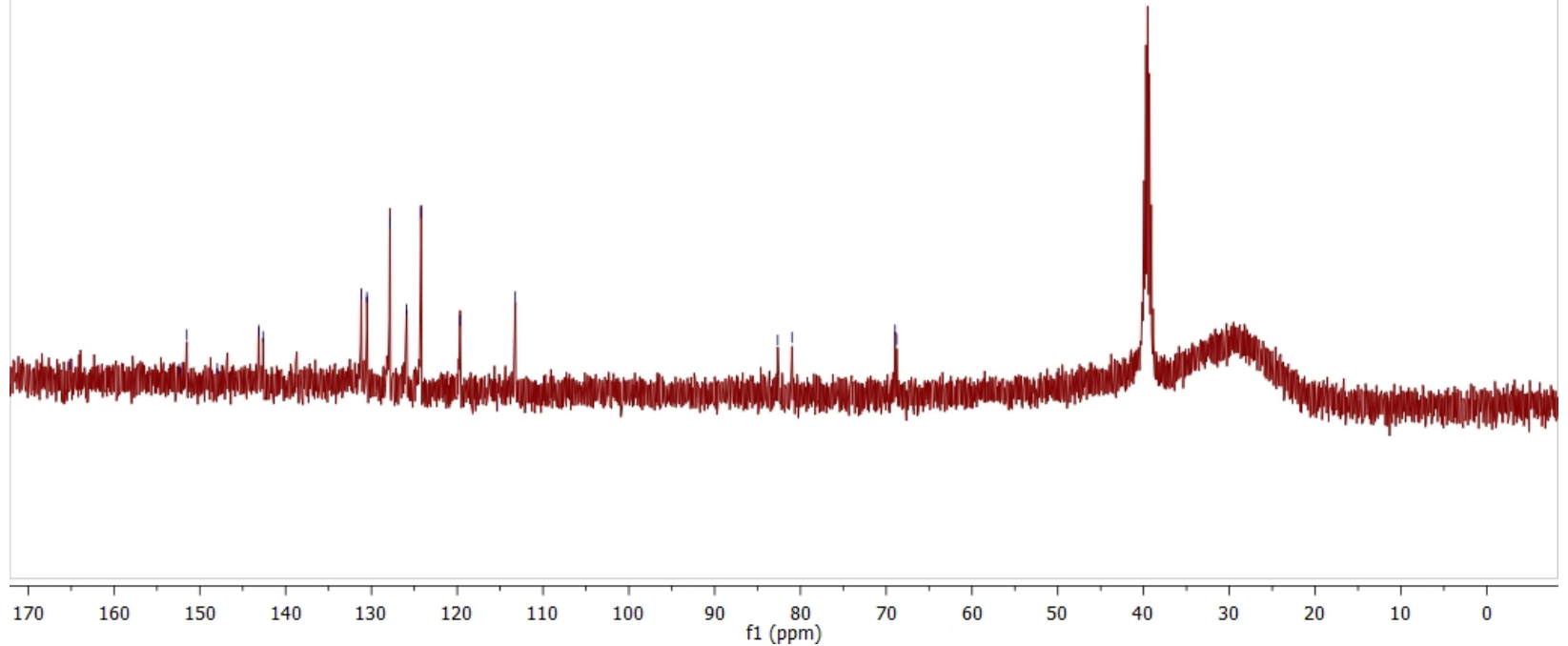




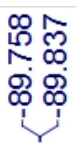
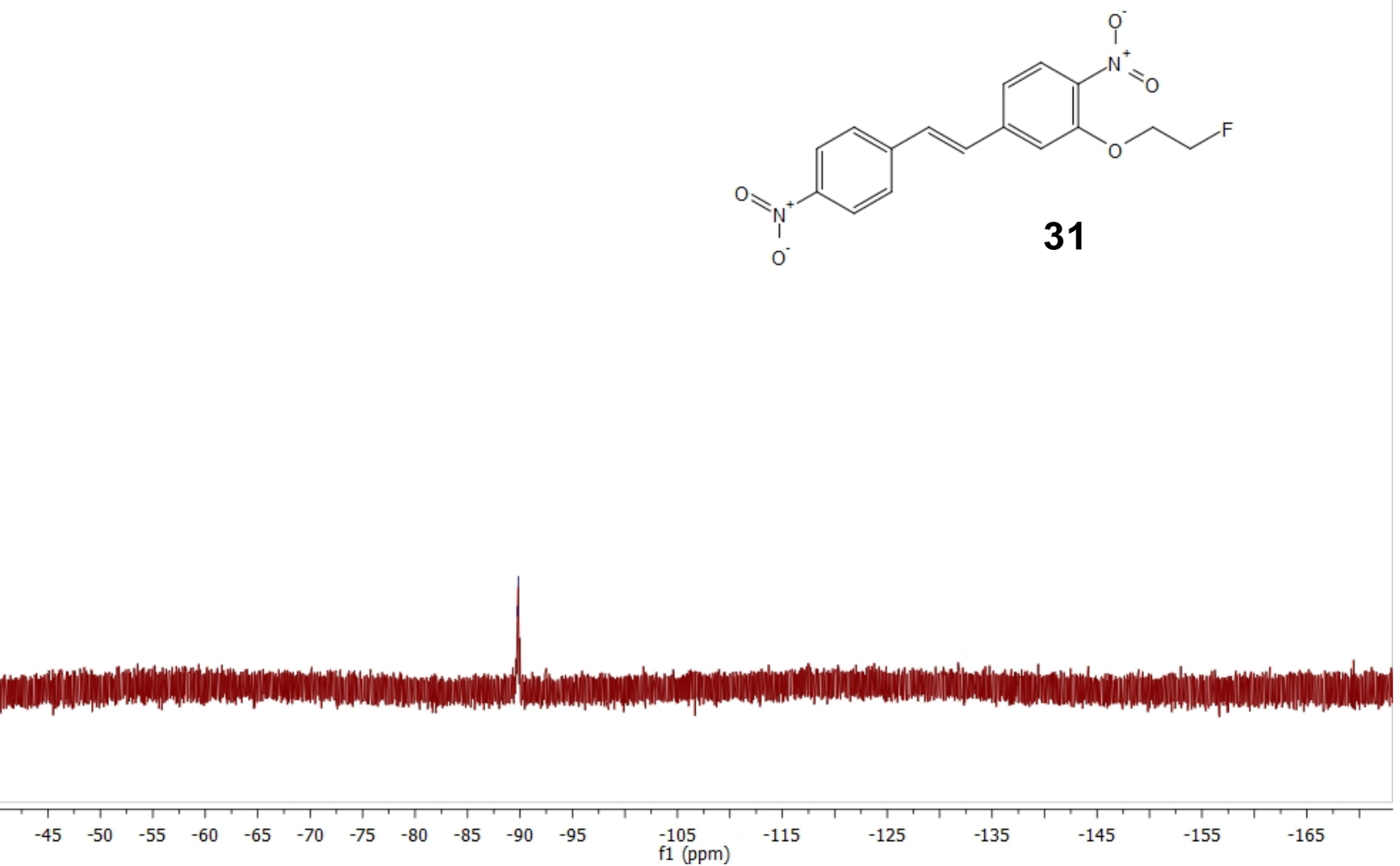


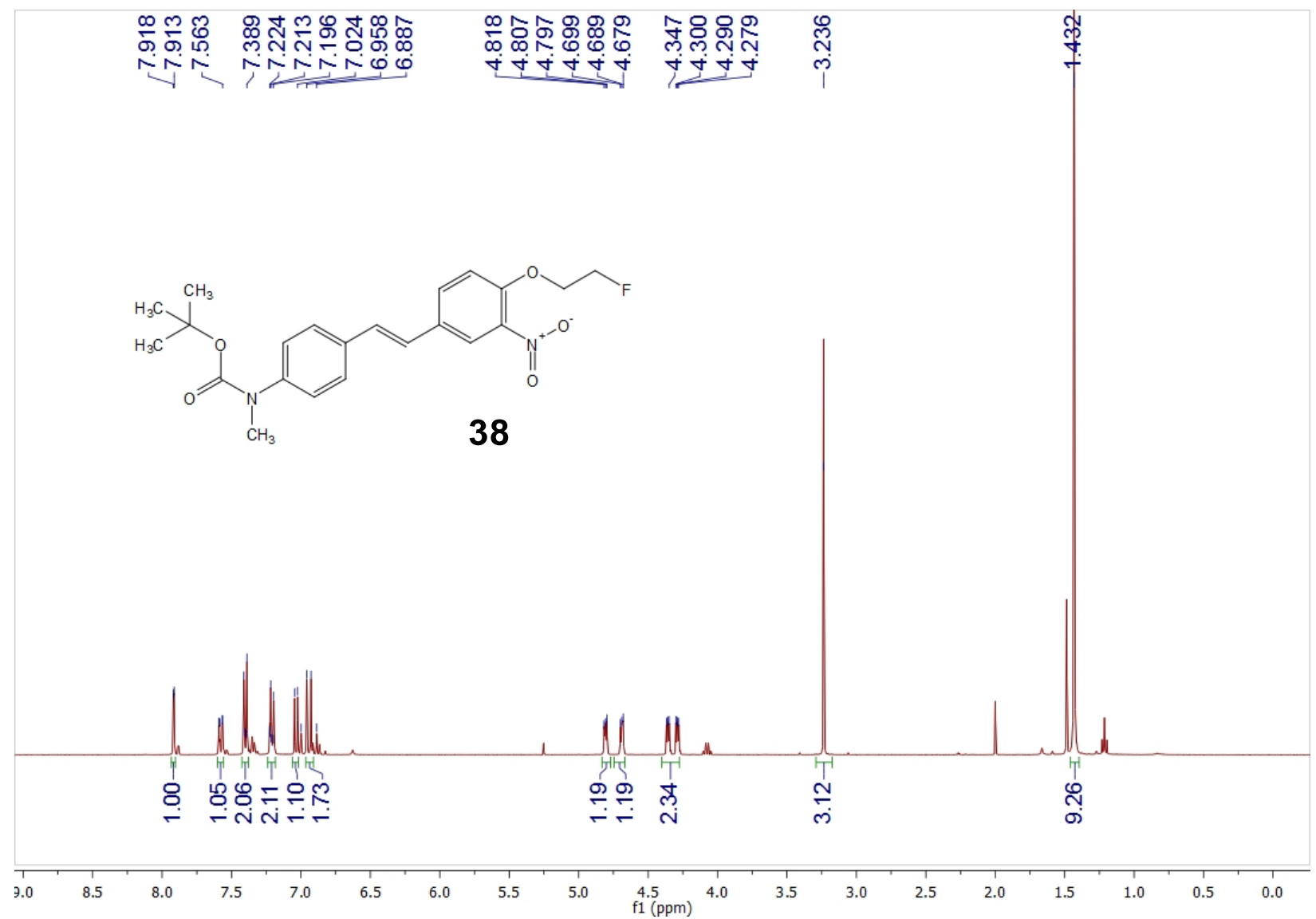




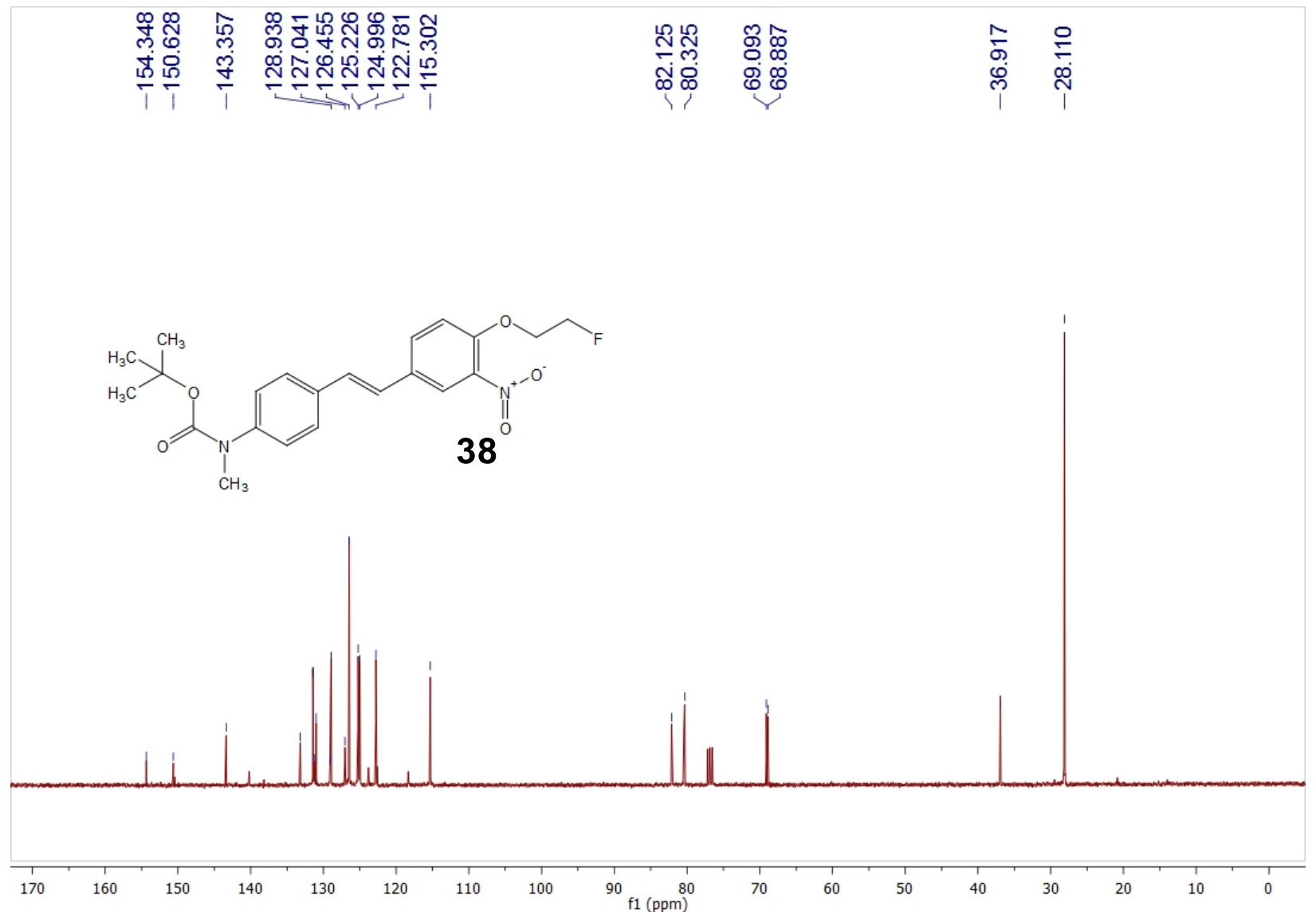




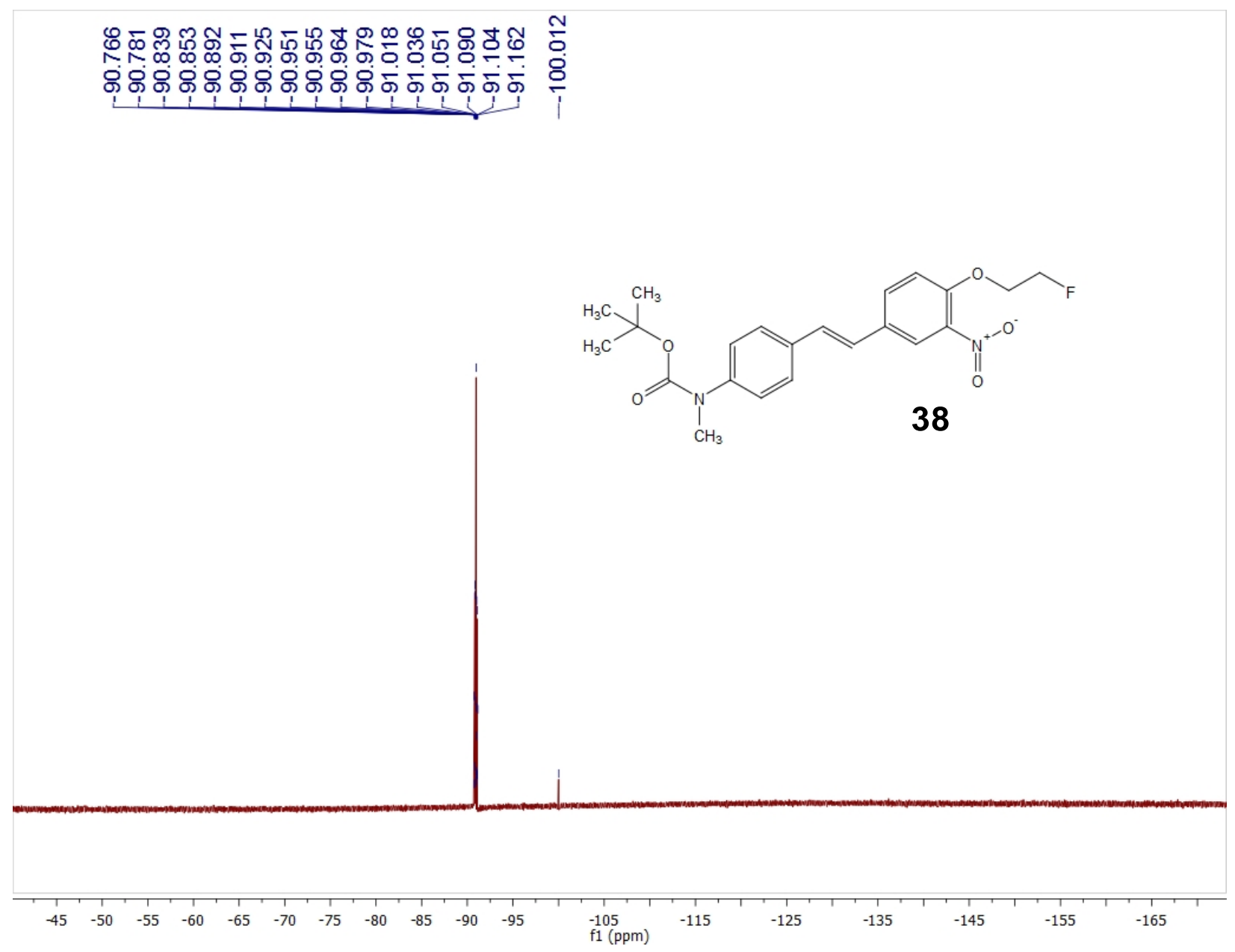




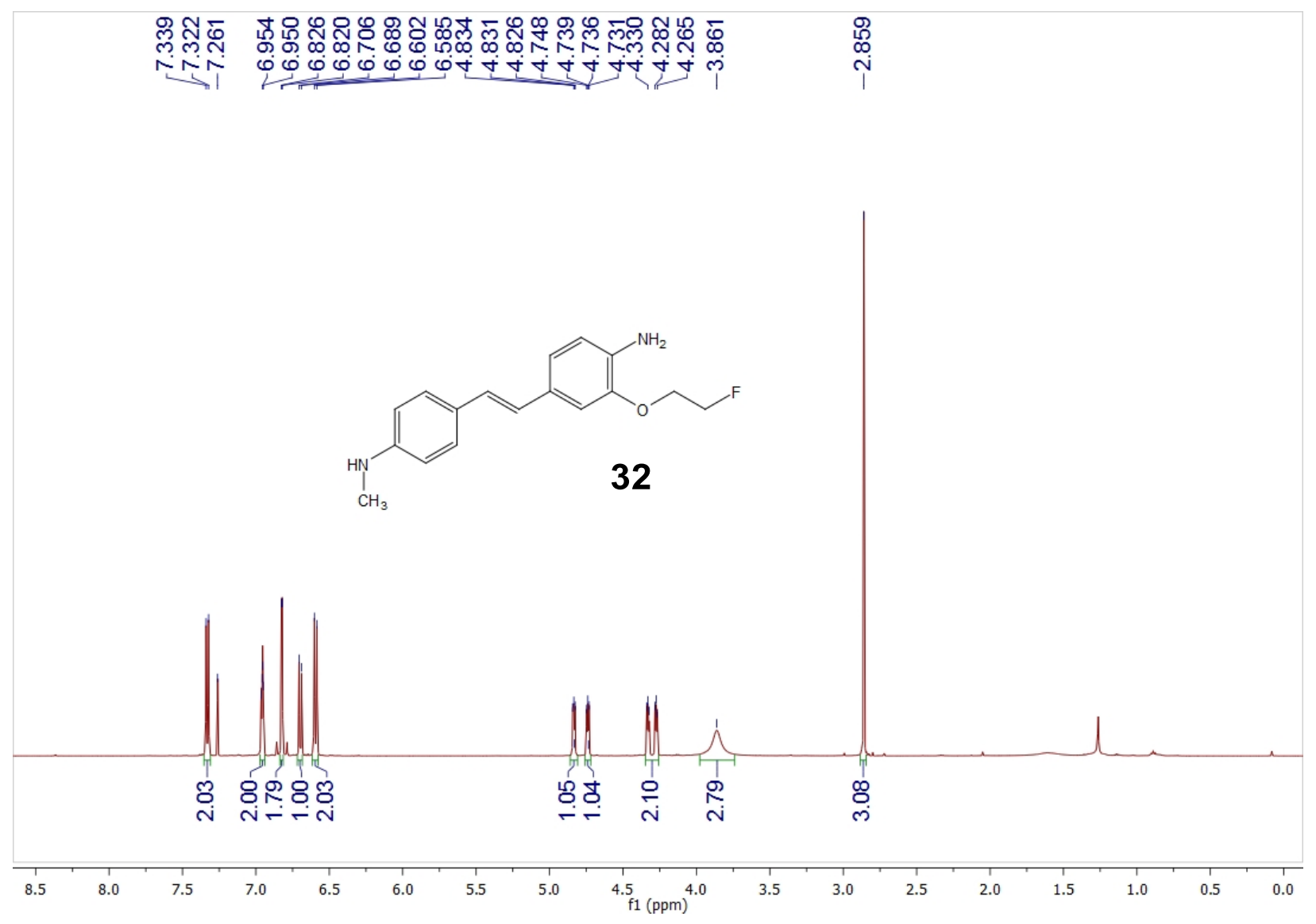




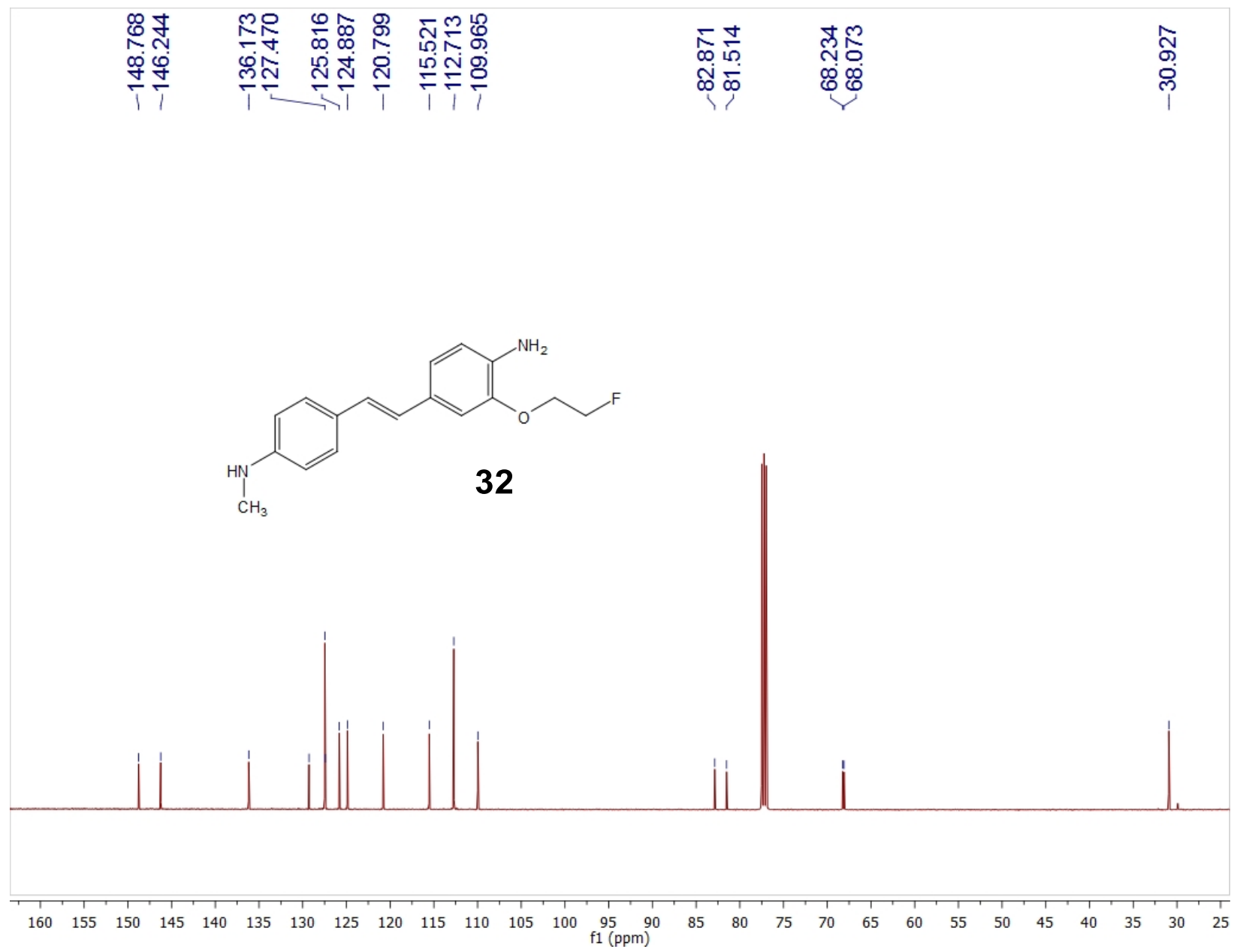




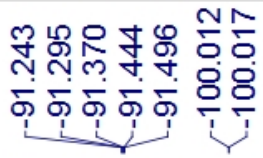
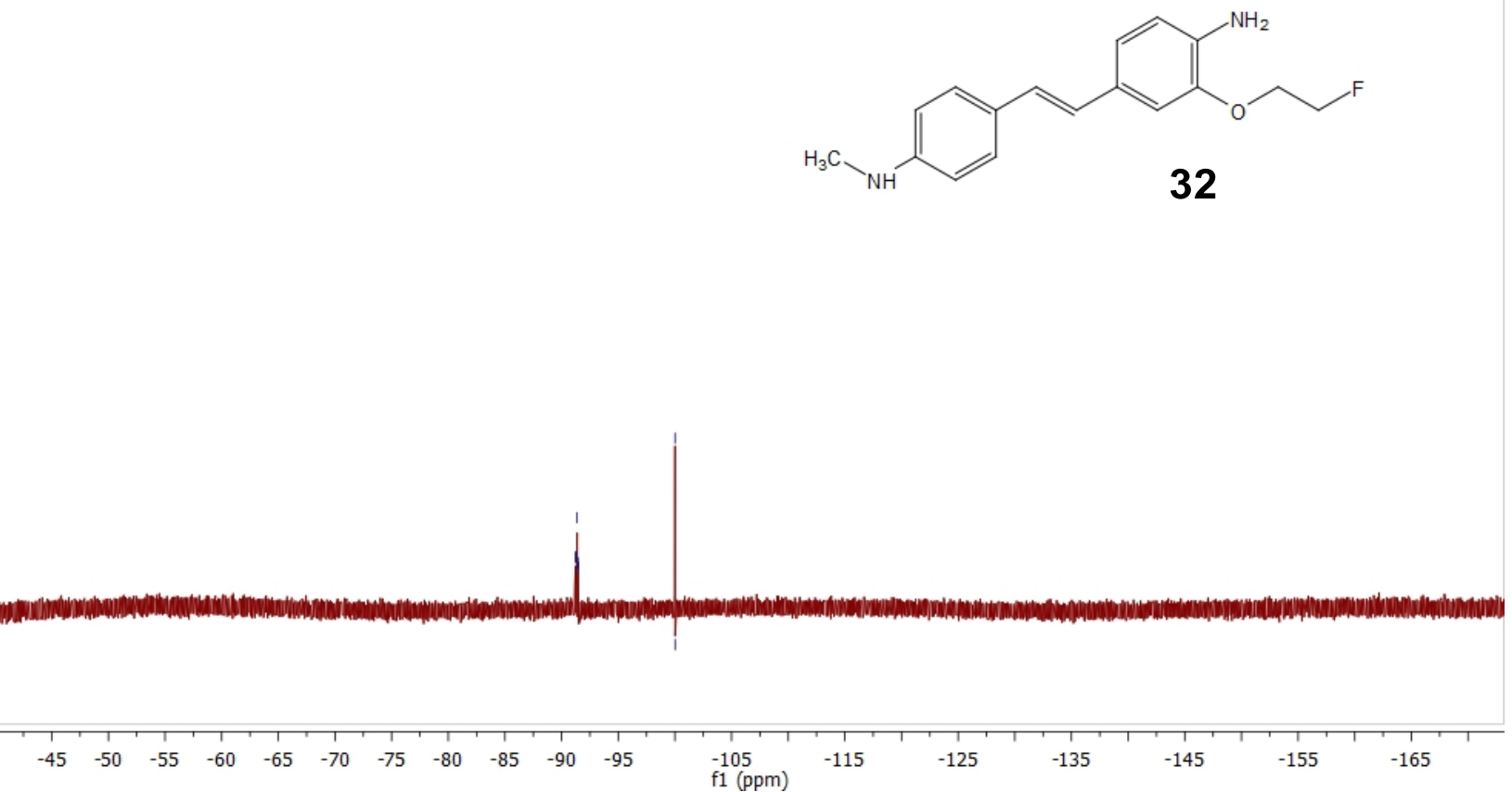


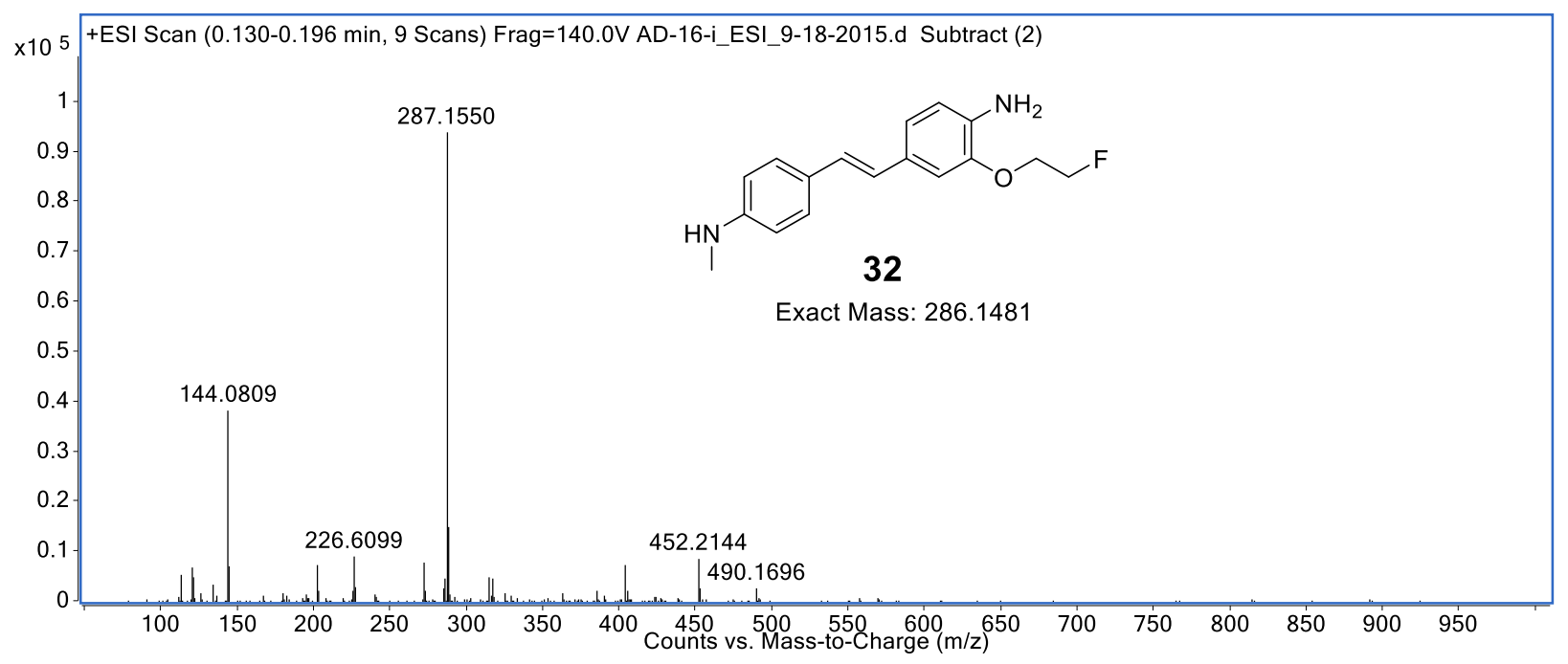


Measurement 10292015_101-1
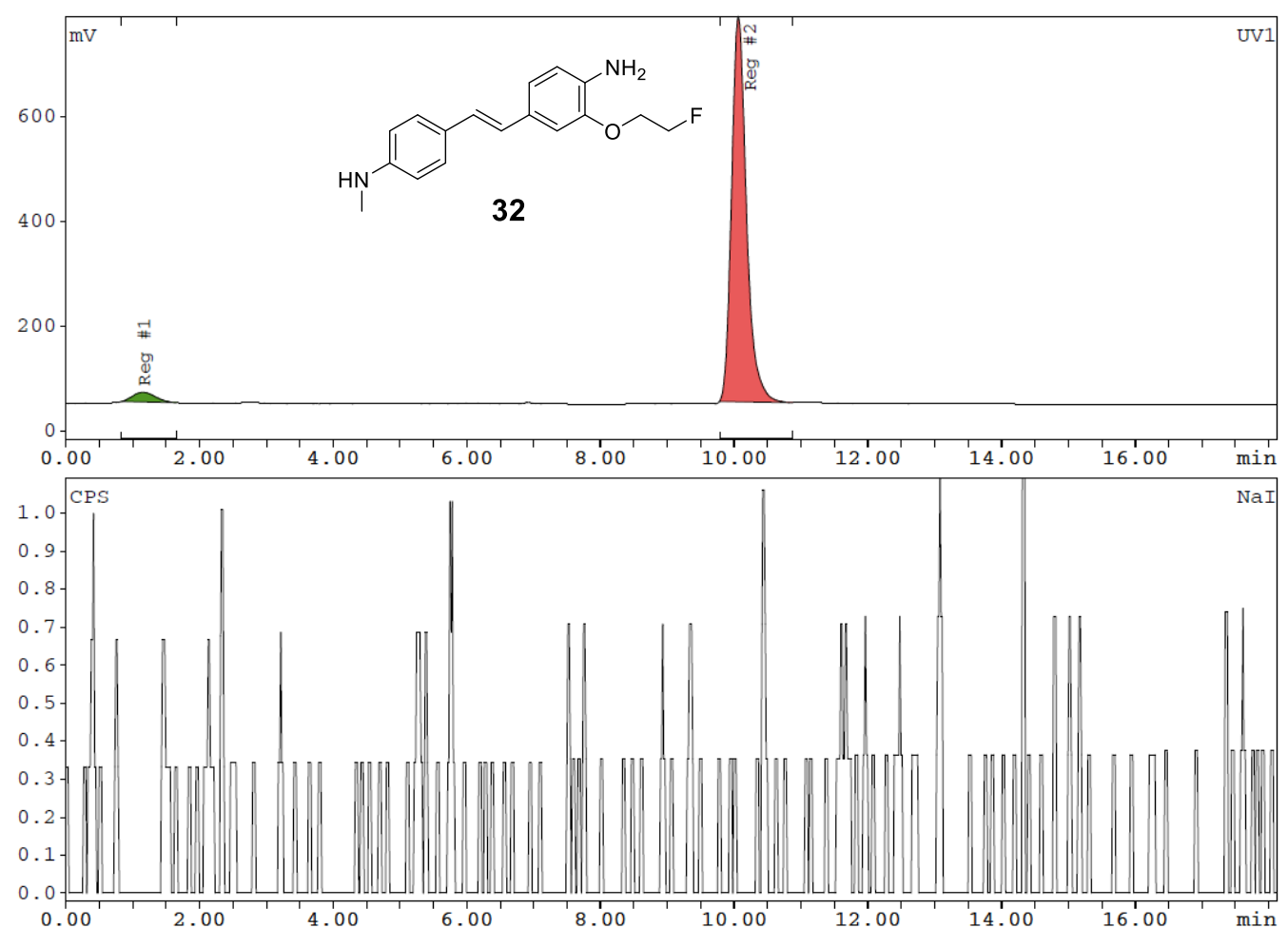

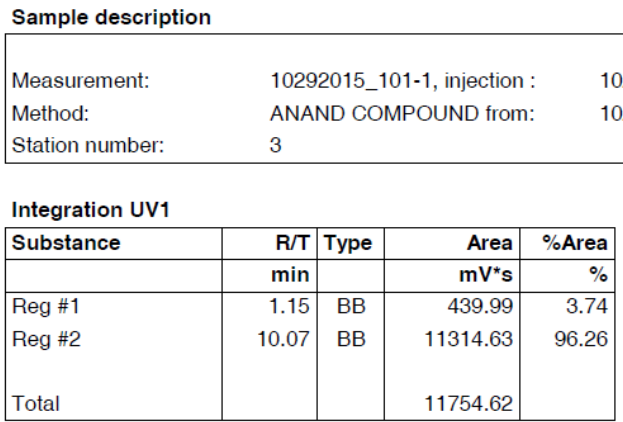


Measurement 07142015_COINJECTION
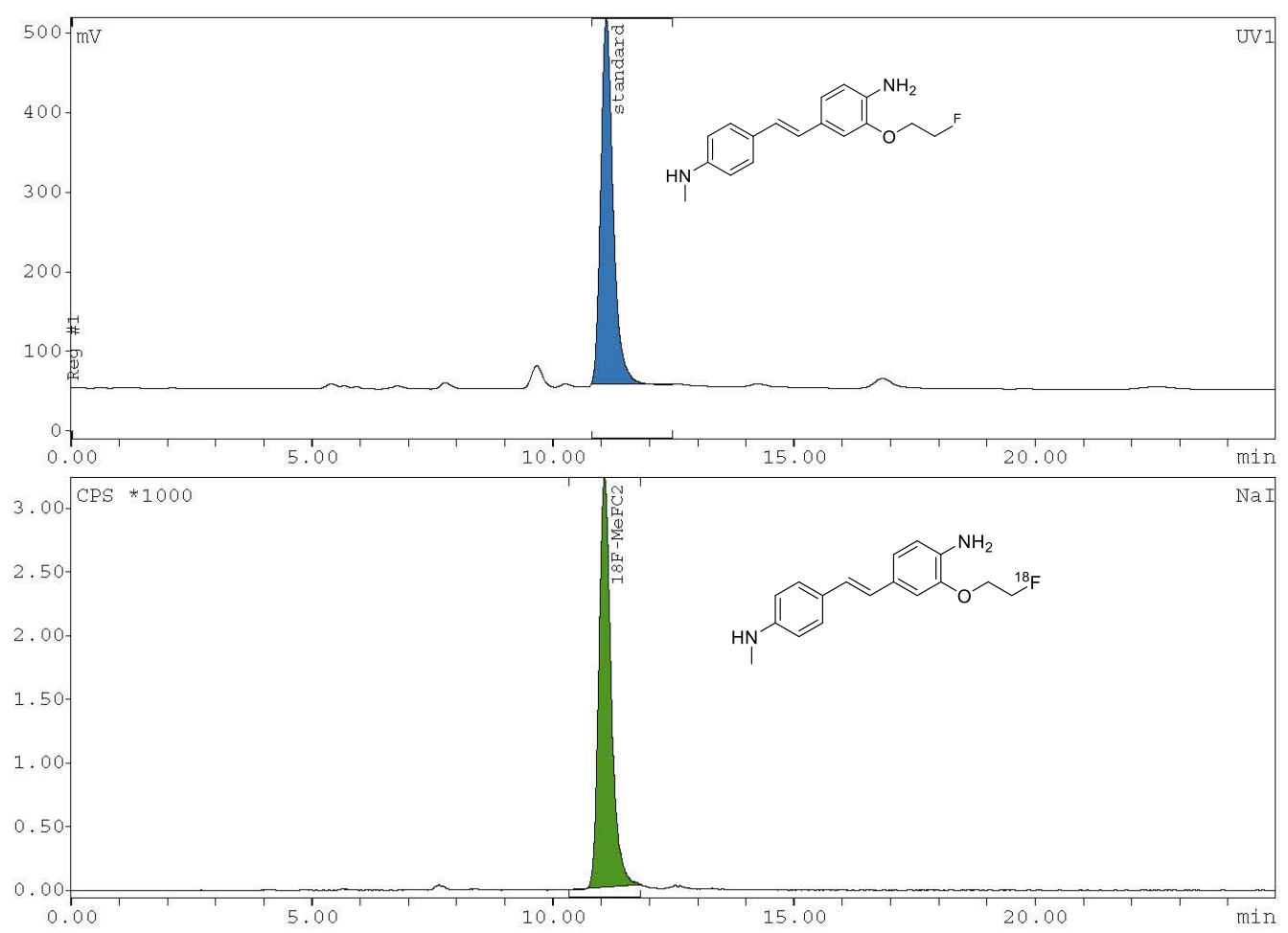

Sample description

\begin{tabular}{llll}
\hline & & & \\
Measurement: & 07142015 COINJECTION, injection : & $7 / 14 / 2015$ & $3: 35$ PM \\
Method: & ANAND COMPOUND from: $\quad 7 / 14 / 2015$ & $3: 31$ PM \\
Station number: & 3 & & \\
\hline
\end{tabular}

Integration Nal
\begin{tabular}{|l|r|r|r|r|}
\hline Substance & R/T & Type & Area & $\%$ Area \\
\hline & min & & Counts & $\%$ \\
\hline 18 F-MeFC2 & 11.07 & BB & 56888.83 & 100.00 \\
& & & & \\
Total & & & 56888.83 & \\
Total area & & & 63371.25 & \\
\hline
\end{tabular}

Integration UV1

\begin{tabular}{|l|r|r|r|r|}
\hline Substance & R/T & Type & Area & \%Area \\
\hline & $\mathbf{m i n}$ & & $\mathbf{m V}^{*} \mathbf{S}$ & $\%$ \\
\hline Reg \#1 & 0.00 & BB & 0.046 & 0.00 \\
standard & 11.10 & BB & 8173.148 & 100.00 \\
& & & & \\
Total & & & 8173.194 & \\
\hline
\end{tabular}



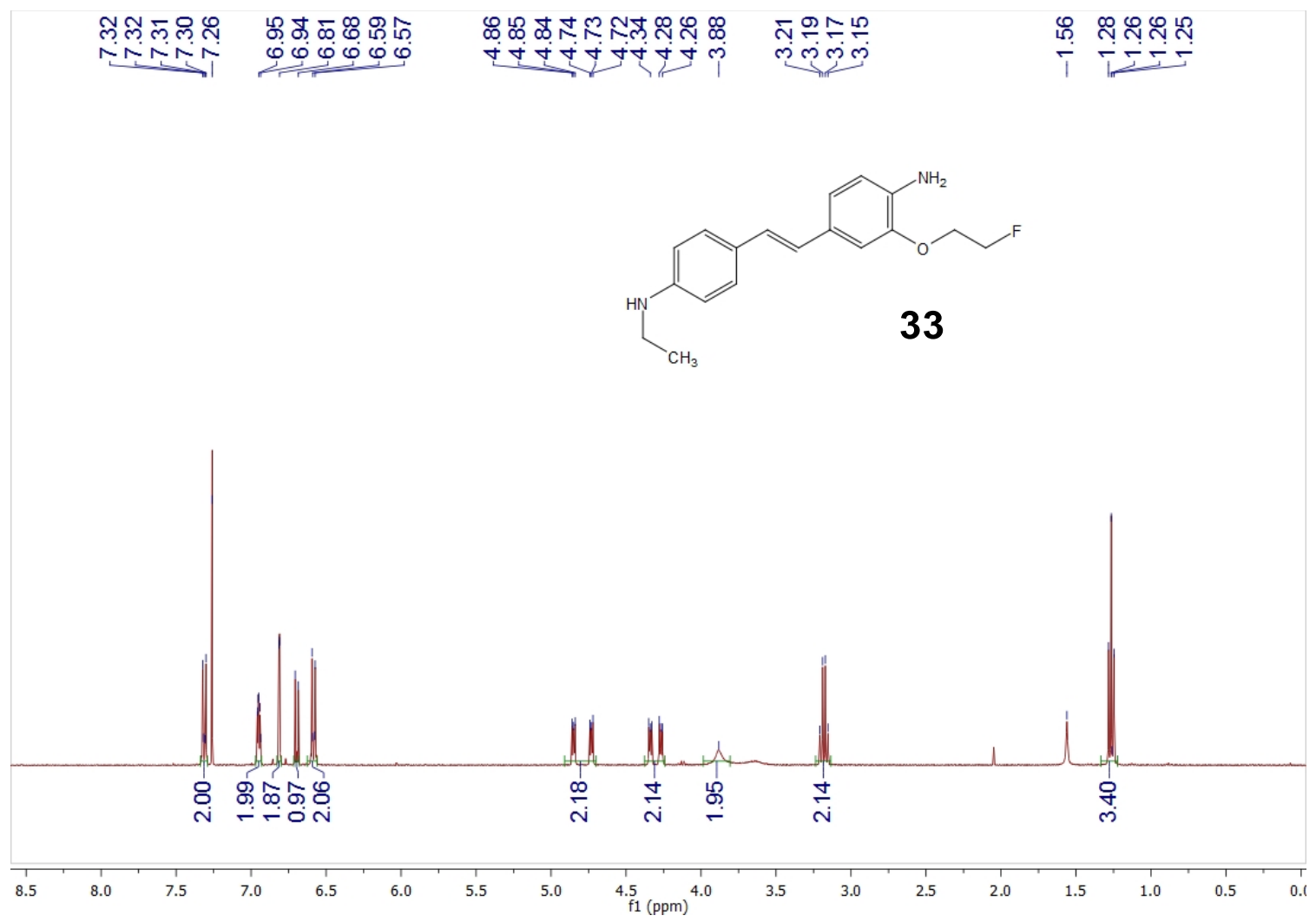


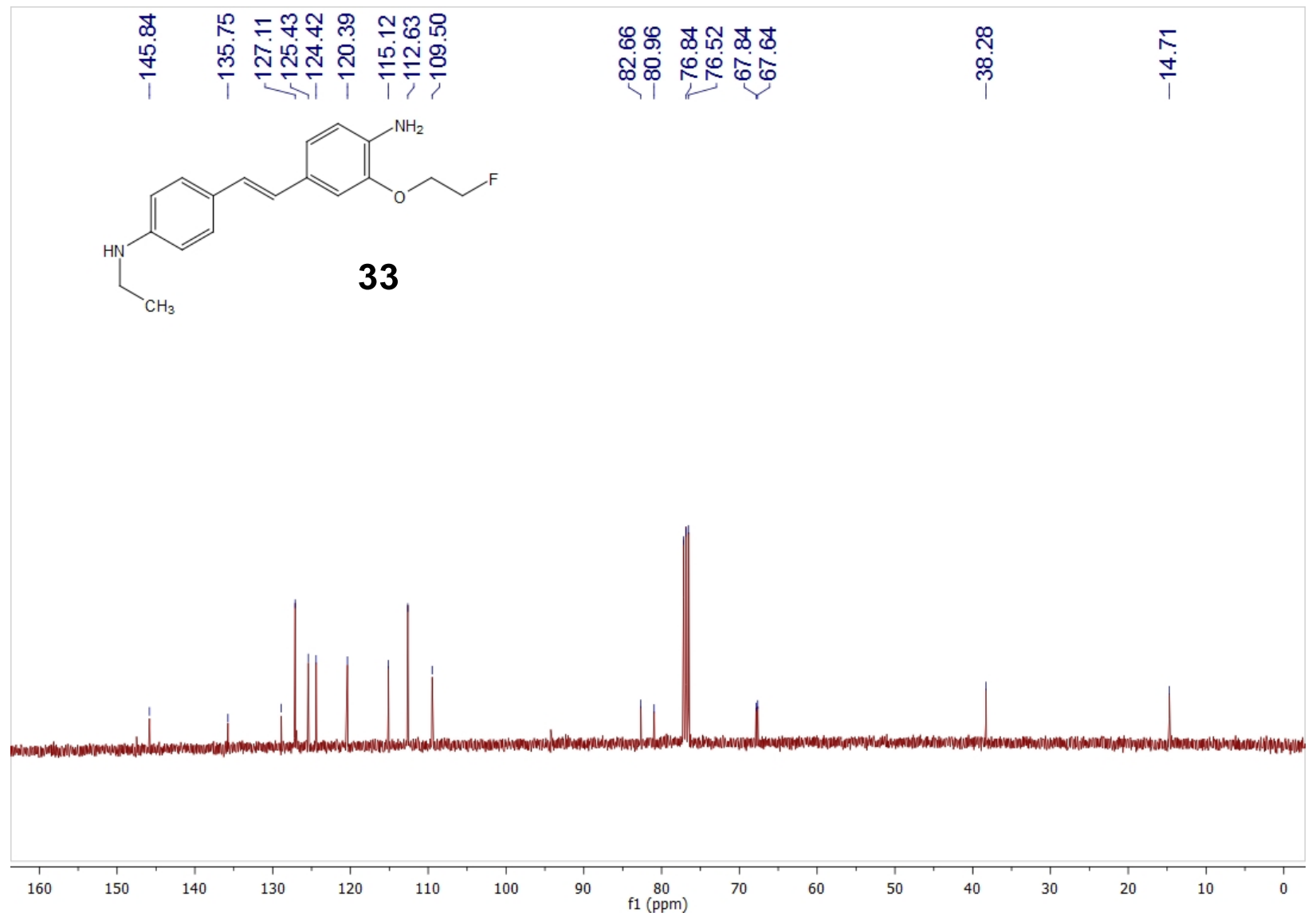




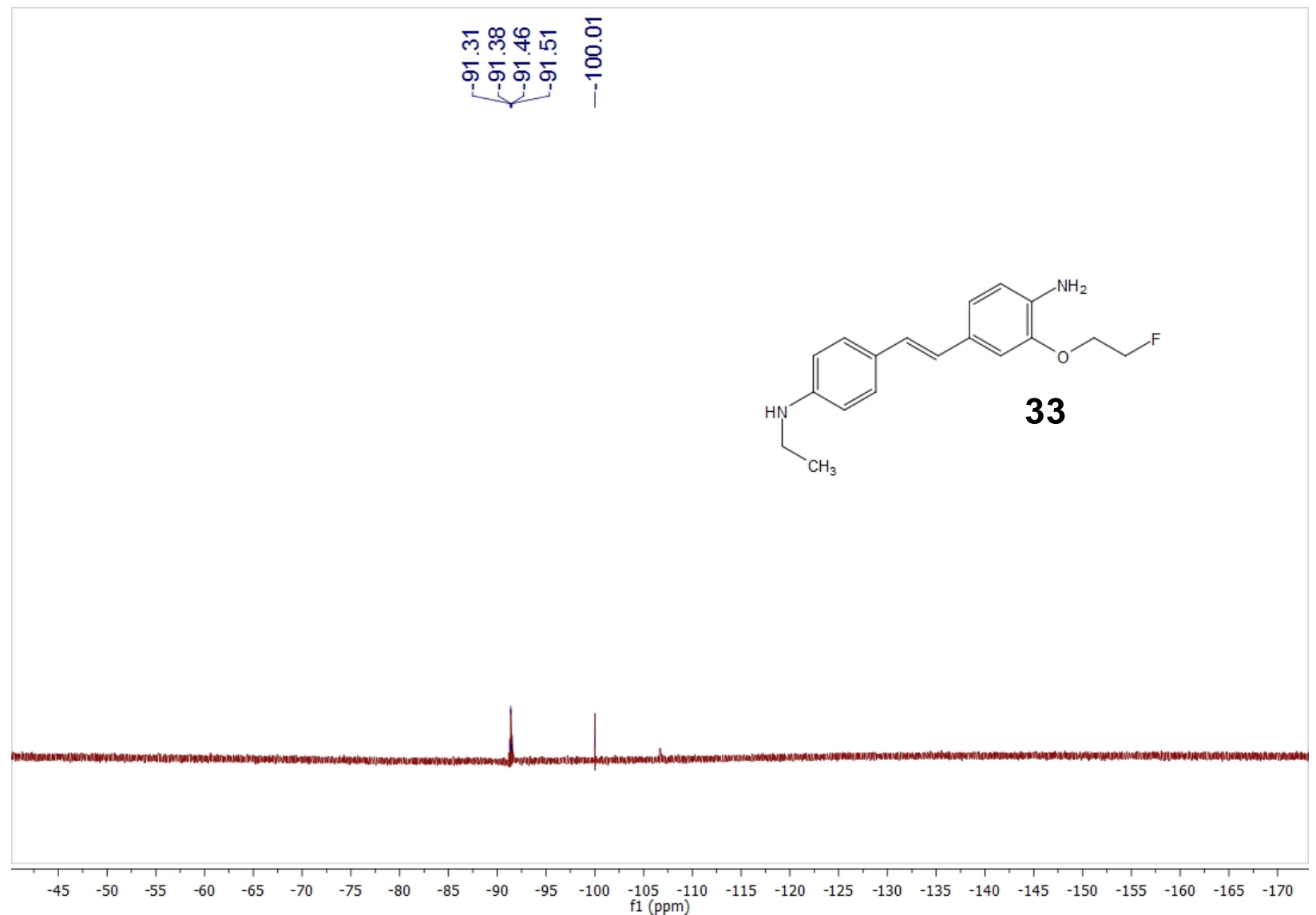




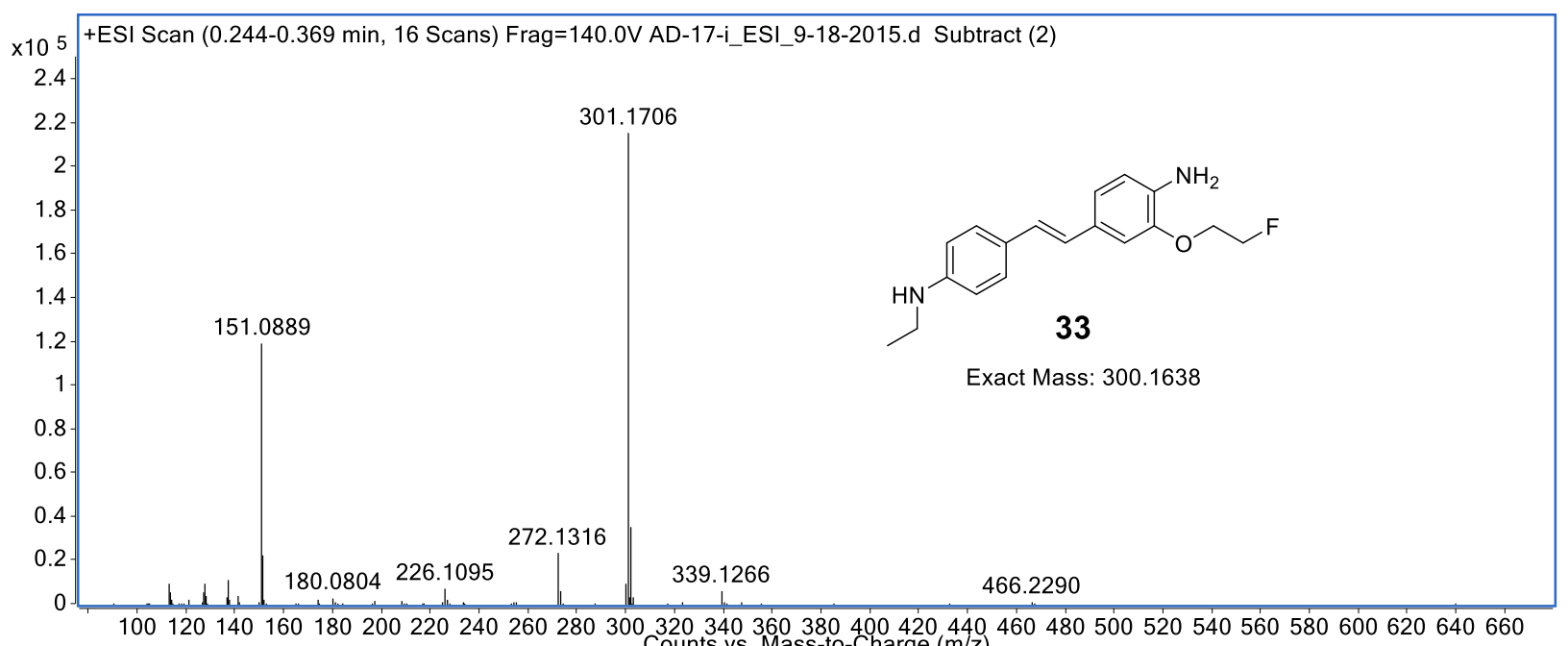

$100120140160180200220240260280300320340 \begin{gathered}360 \\ \text { Counts vs. Mass-to-Charge }(\mathrm{m} / \mathrm{z})\end{gathered} 460480500520540560580600620640660$ 

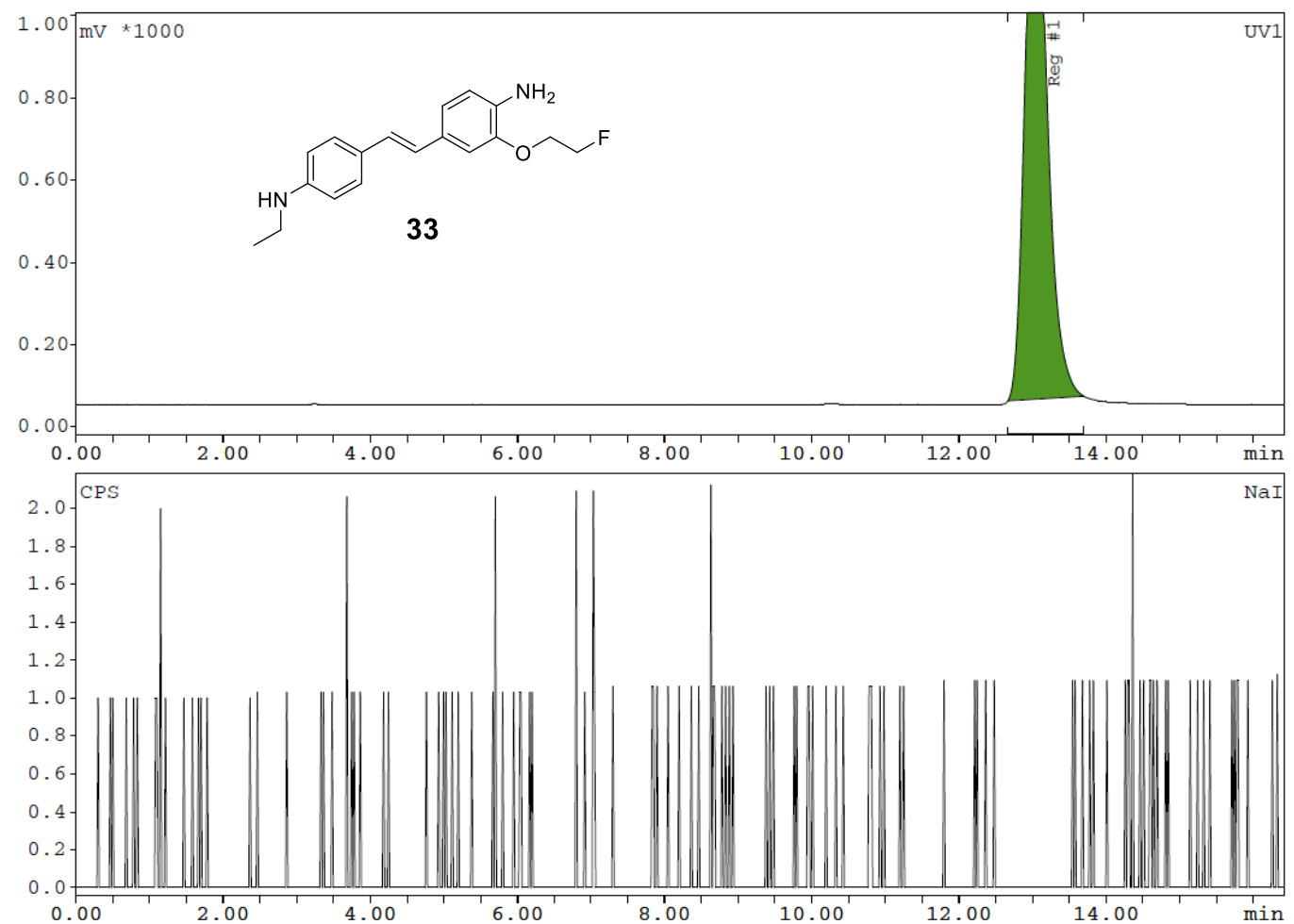

Sample description

\begin{tabular}{|lll|}
\hline & & \\
Measurement: & 09092015_141C_1, injection : & 9/9/2015 $3: 18 \mathrm{PM}$ \\
Method: & ANAND COMPOUND from: & 9/9/2015 2:51 PM \\
Station number: & 3 & \\
\hline
\end{tabular}

Integration UV1

\begin{tabular}{|l|r|r|r|r|}
\hline Substance & $\mathbf{R} / \mathbf{T}$ & Type & Area & $\%$ Area \\
\hline Reg \#1 & $\mathbf{m i n}$ & & $\mathbf{m V}^{*} \mathbf{s}$ & $\%$ \\
\hline Total & 13.12 & BB & 24732.03 & 100.00 \\
\hline
\end{tabular}



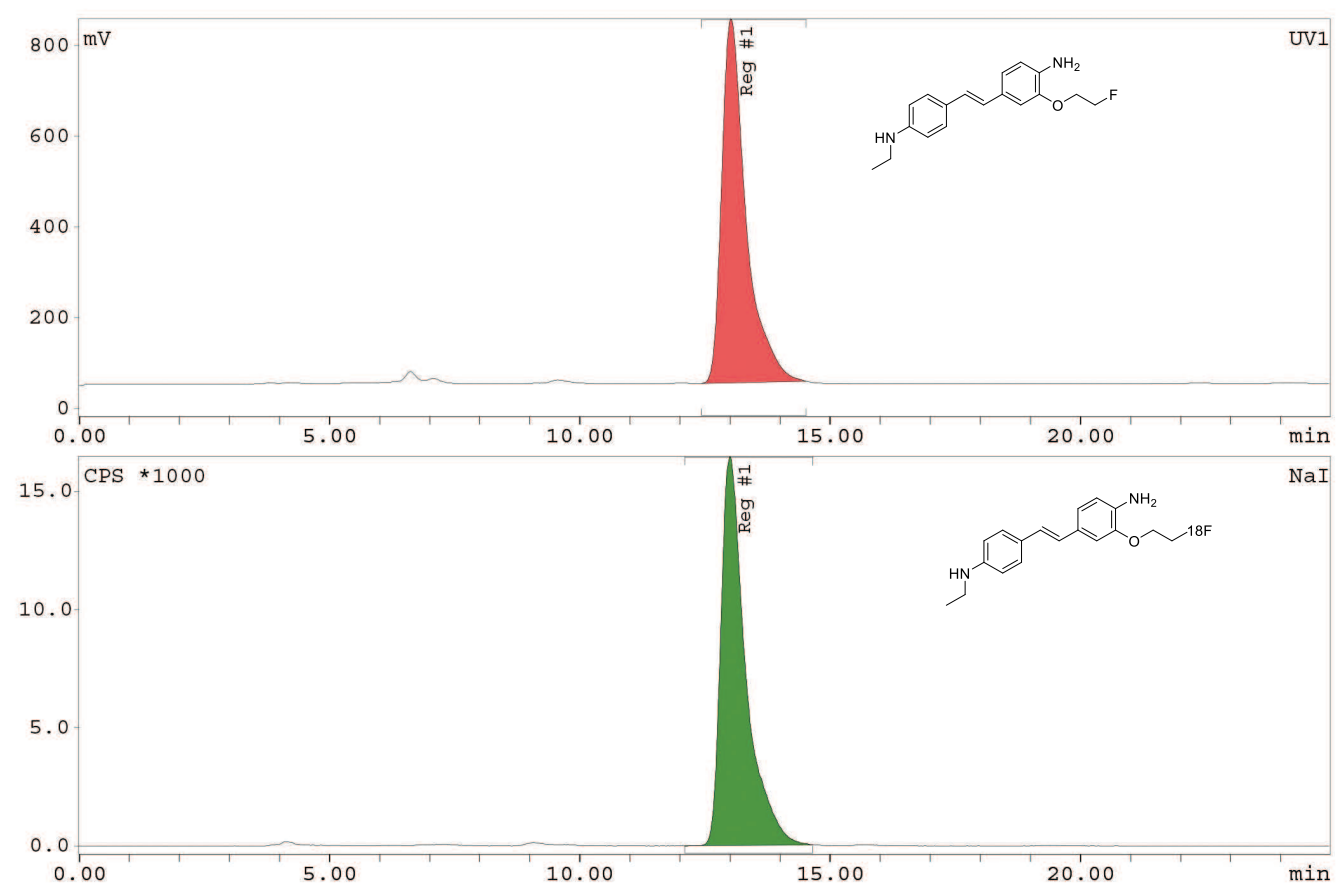

Sample description

\begin{tabular}{llll} 
Measurement: & 07152015_STA_87-2, injection : & $7 / 15 / 2015$ & $3: 04$ \\
Method: & ANAND COMPOUND from: & $7 / 15 / 2015$ & $3: 03$ \\
\hline & & PM
\end{tabular}

Station number:

\begin{tabular}{|l|r|r|r|r|}
\hline Integration Nal & \multicolumn{3}{|r|}{} \\
\hline Substance & R/T & Type & Area & $\%$ Area \\
\hline Reg \#1 & min & & Counts & $\%$ \\
\hline Total & 13.02 & BB & 565083.6 & 100.00 \\
\hline Total area & & & & \\
\hline
\end{tabular}

Integration UV1

\begin{tabular}{|l|r|r|r|r|}
\hline Substance & R/T & Type & Area & \%Area \\
\hline Reg \#1 & min & & $\mathbf{m V}^{*} \mathbf{s}$ & $\%$ \\
\hline Total & 13.02 & BB & 26889.87 & 100.00 \\
\hline
\end{tabular}




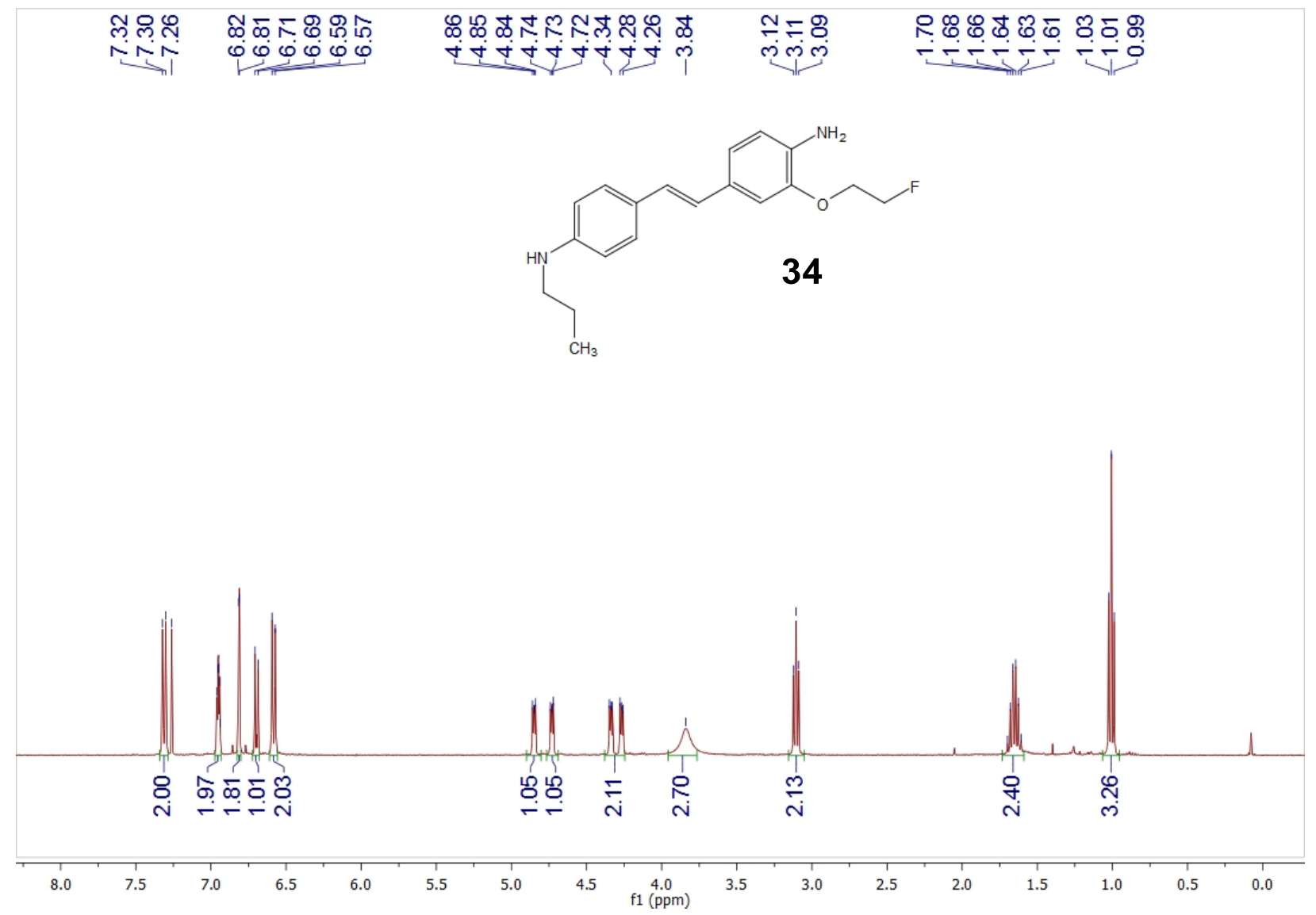




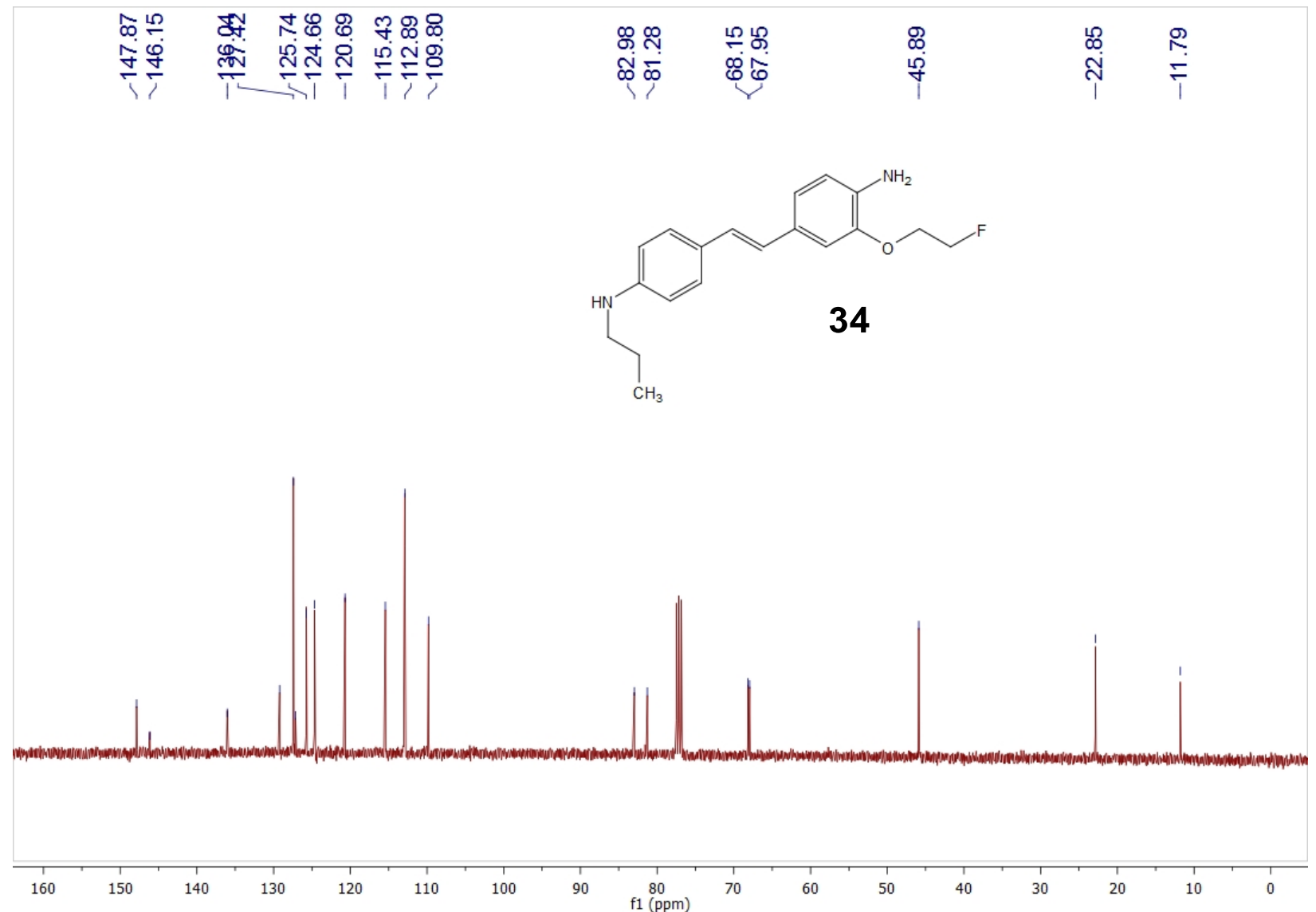



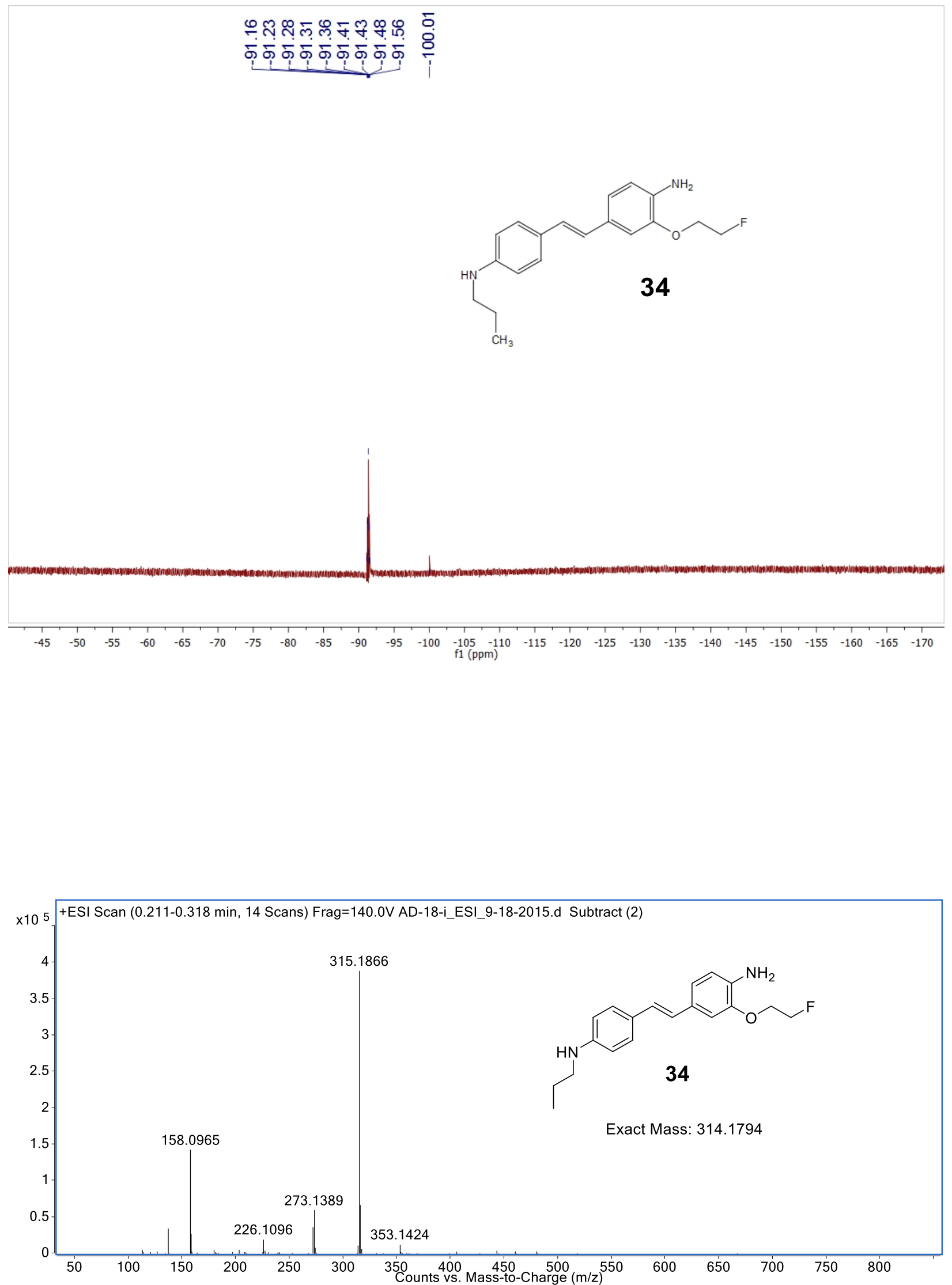
Measurement 09092015_134C_1
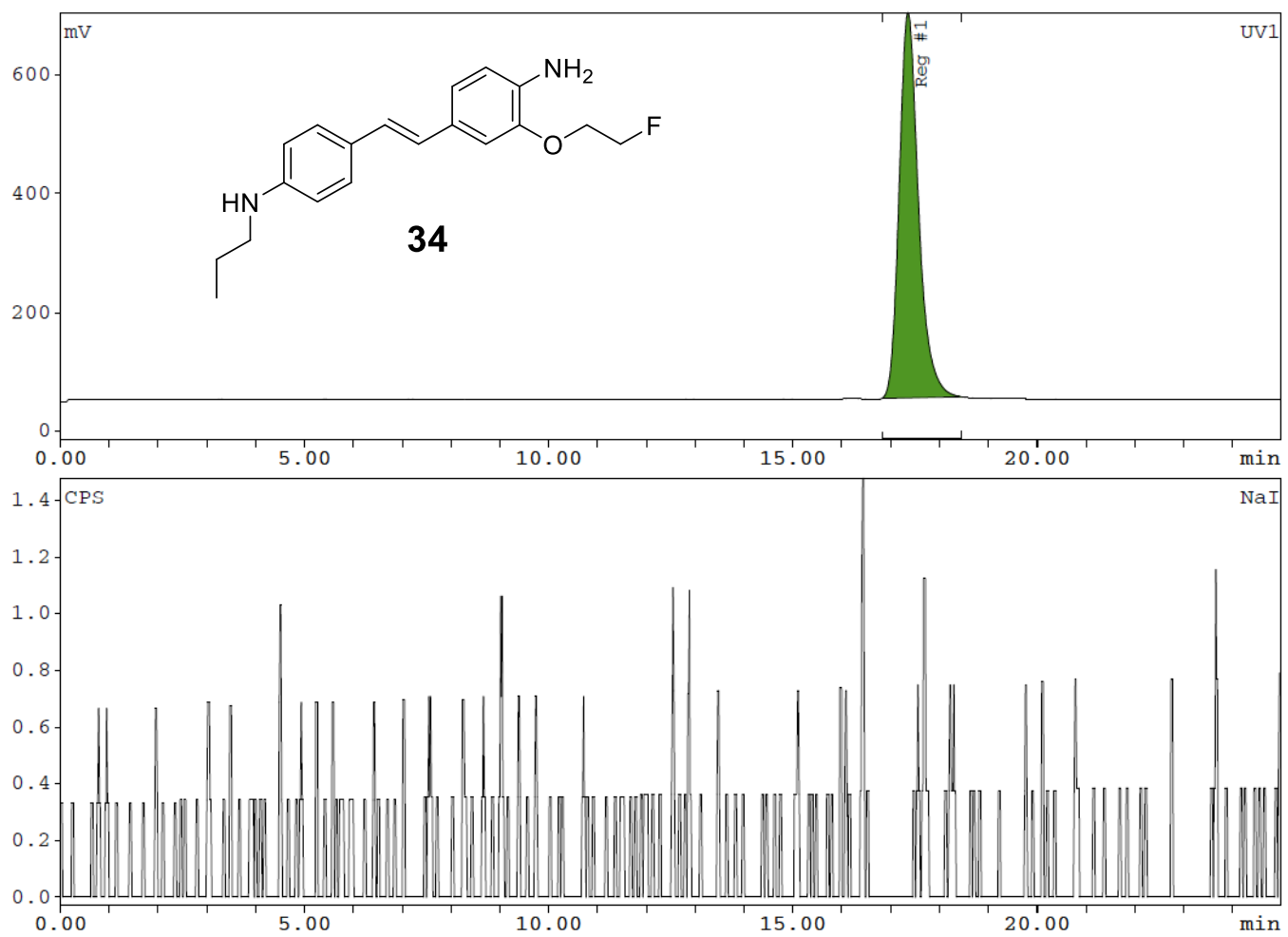

Sample description

Measurement: $\quad$ 09092015_134C_1, injection : 9/9/2015 4:34 PM

Method: $\quad$ ANAND COMPOUND from: 9/9/2015 2:51 PM

Station number:

3

Integration UV1
\begin{tabular}{|l|r|r|r|r|}
\hline Substance & R/T & Type & Area & $\%$ Area \\
\hline & min & & mV*s $^{*}$ & $\%$ \\
\hline Reg \#1 & 17.37 & BB & 17671.21 & 100.00 \\
Total & & & & \\
\hline
\end{tabular}



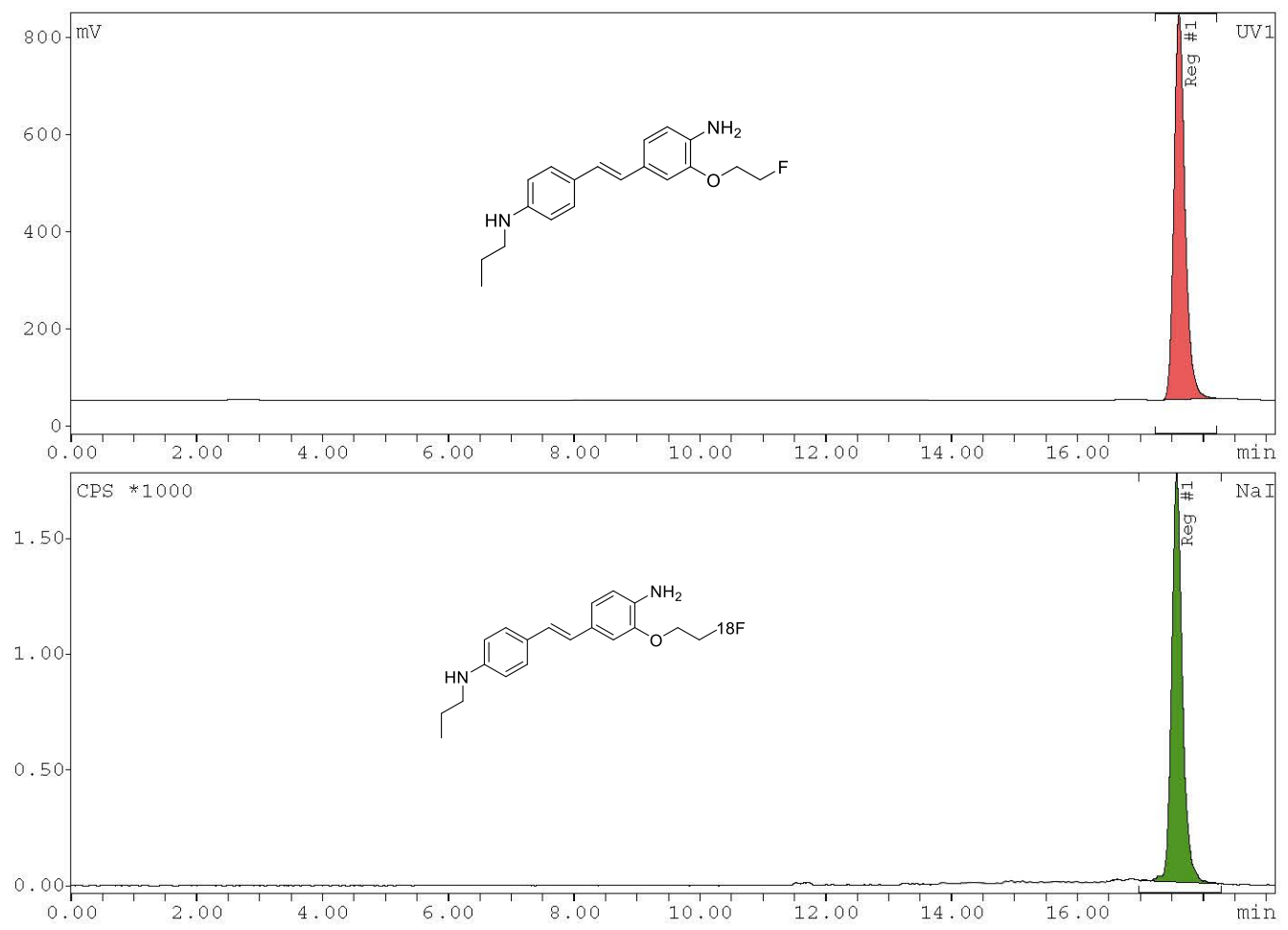

Sample description

\begin{tabular}{llll|}
\hline & & & \\
Measurement: & $01262016 \_37$, injection : & $1 / 26 / 2016$ & $1: 05 \mathrm{PM}$ \\
Method: & ANAND COMPOUND from: & $1 / 26 / 2016$ & $1: 01 \mathrm{PM}$ \\
Station number: & 3 & & \\
\hline
\end{tabular}

Timetable

\begin{tabular}{|r|r|l|r|r|l|}
\hline No. & Time, $\mathbf{m}$ & Event type & Value & Unit & Comment \\
\hline 1 & 17.35 & Gradient & Hold & & \\
2 & 19.07 & Gradient & Hold & & \\
3 & 19.15 & Manual stop & Stp & & \\
\hline
\end{tabular}

Integration Nal

\begin{tabular}{|l|r|r|r|r|}
\hline Substance & R/T & Type & Area & \%Area \\
\hline & min & & Counts & $\%$ \\
\hline Reg\#1 & 17.58 & BB & 20727.27 & 100.00 \\
& & & & \\
Total & & & 20727.27 & \\
Total area & & & 32113.31 & \\
\hline
\end{tabular}

Integration UV1

\begin{tabular}{|l|r|r|r|r|}
\hline Substance & R/T & Type & Area & \%Area \\
\hline & $\mathbf{m i n}$ & & $\mathbf{m V *}$ & $\%$ \\
\hline Reg\#1 & 17.62 & BB & 9626.580 & 100.00 \\
Total & & & & \\
\hline
\end{tabular}




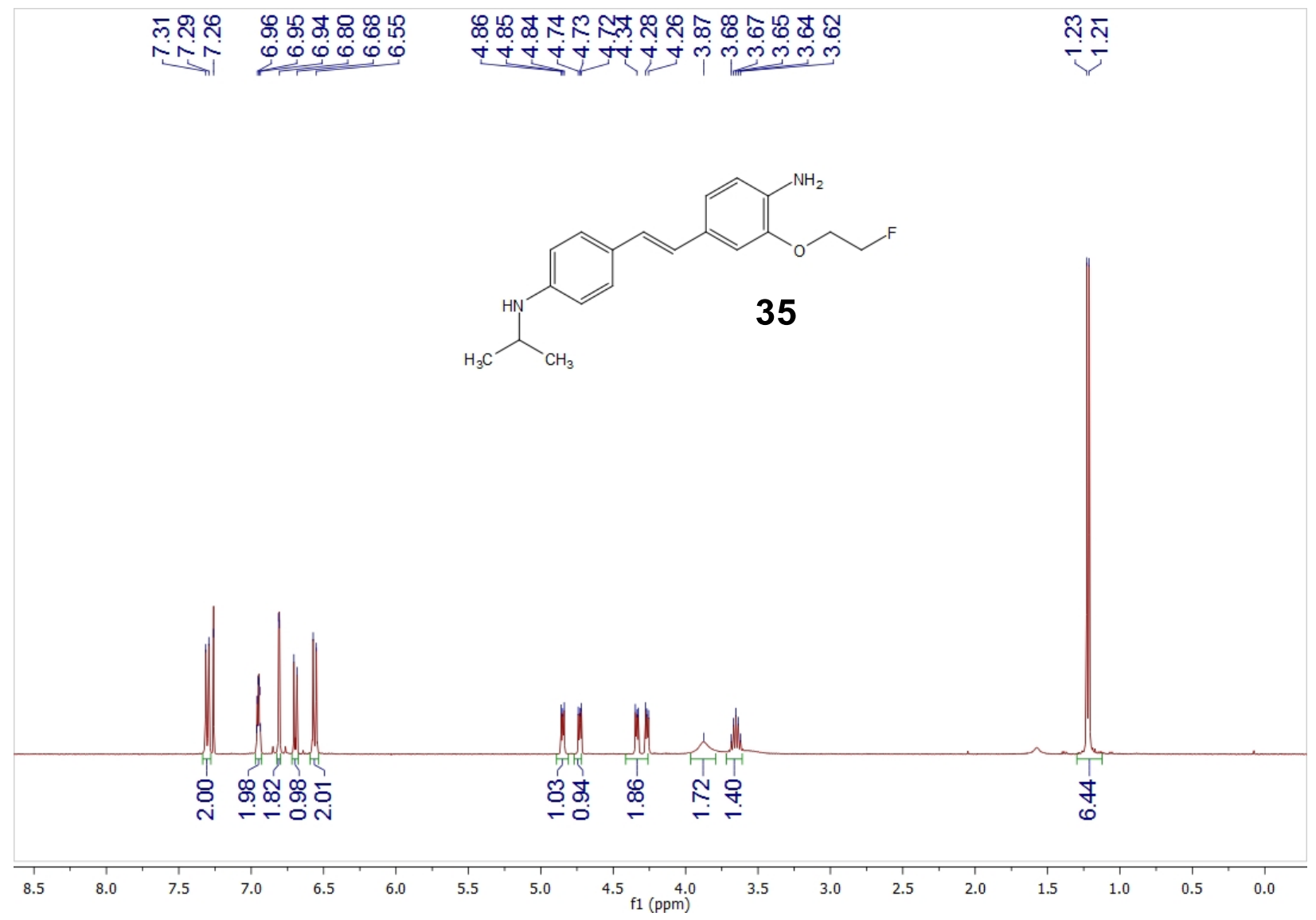




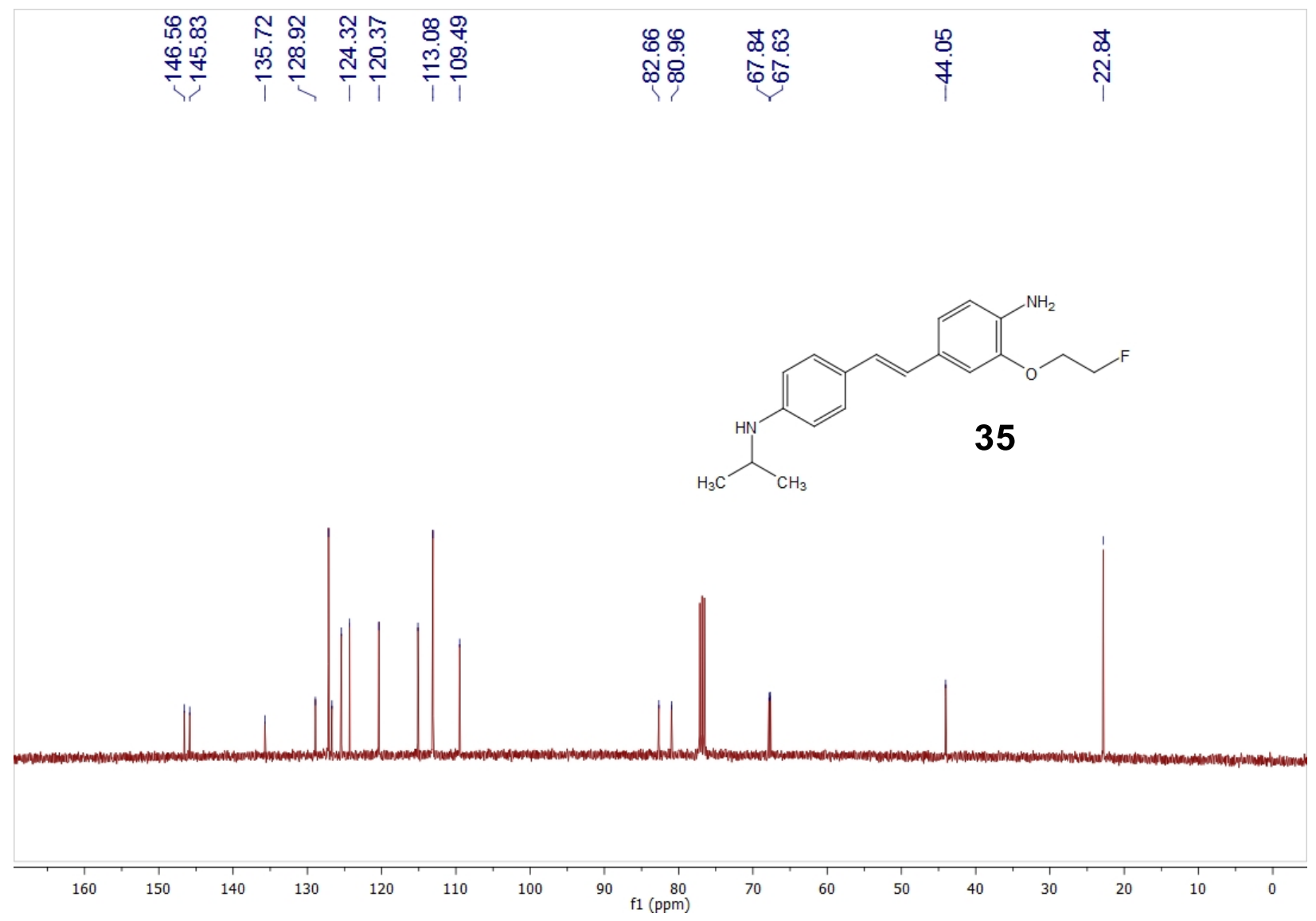




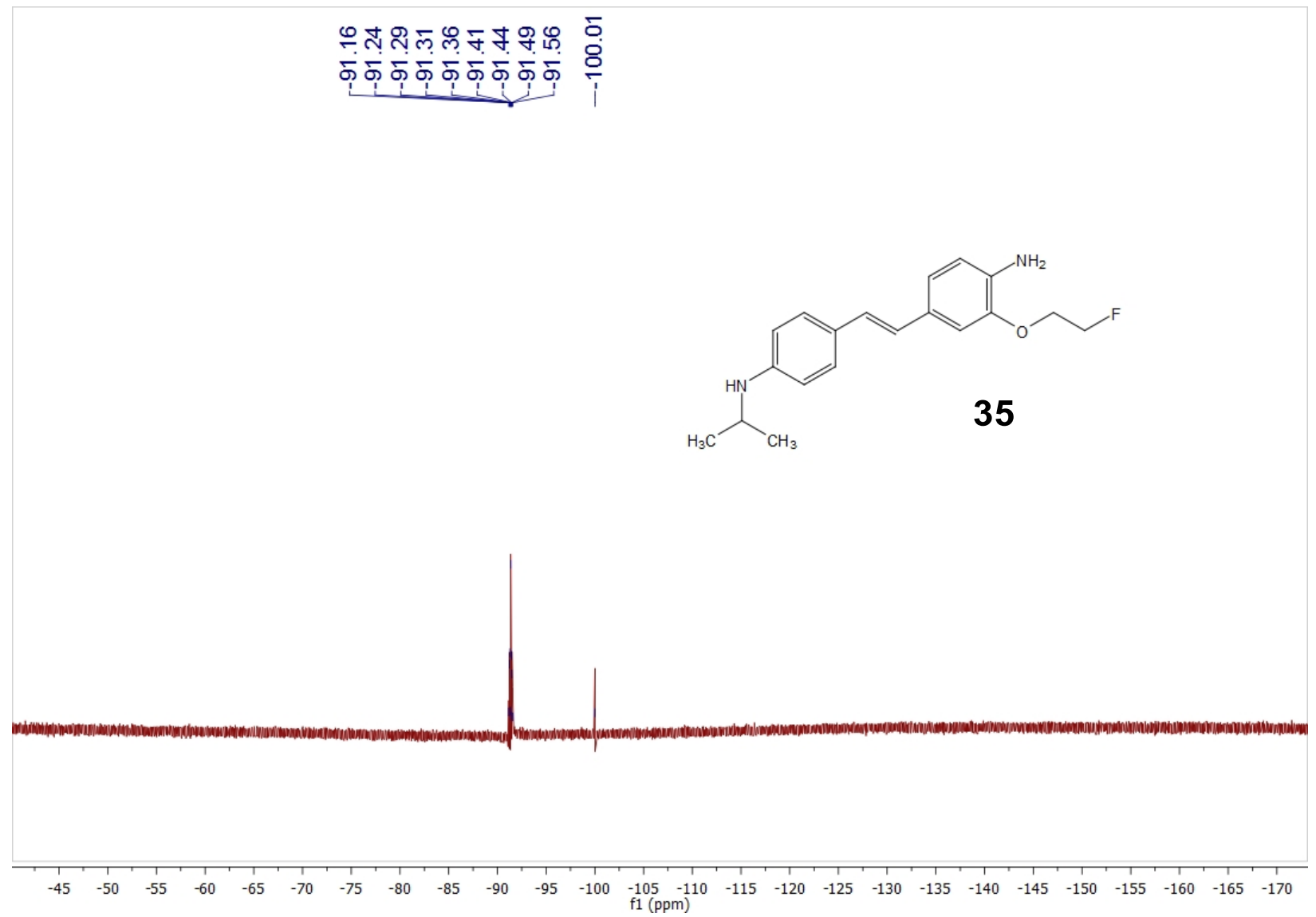




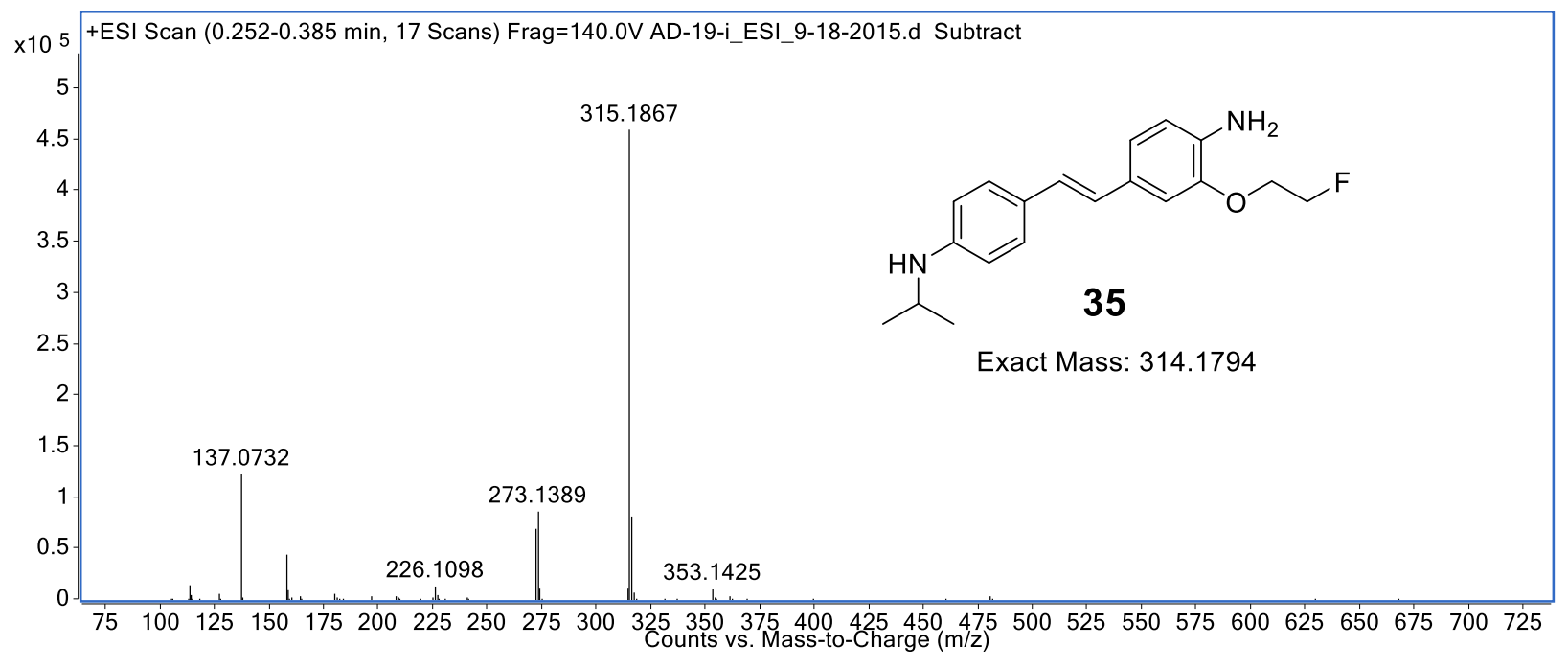



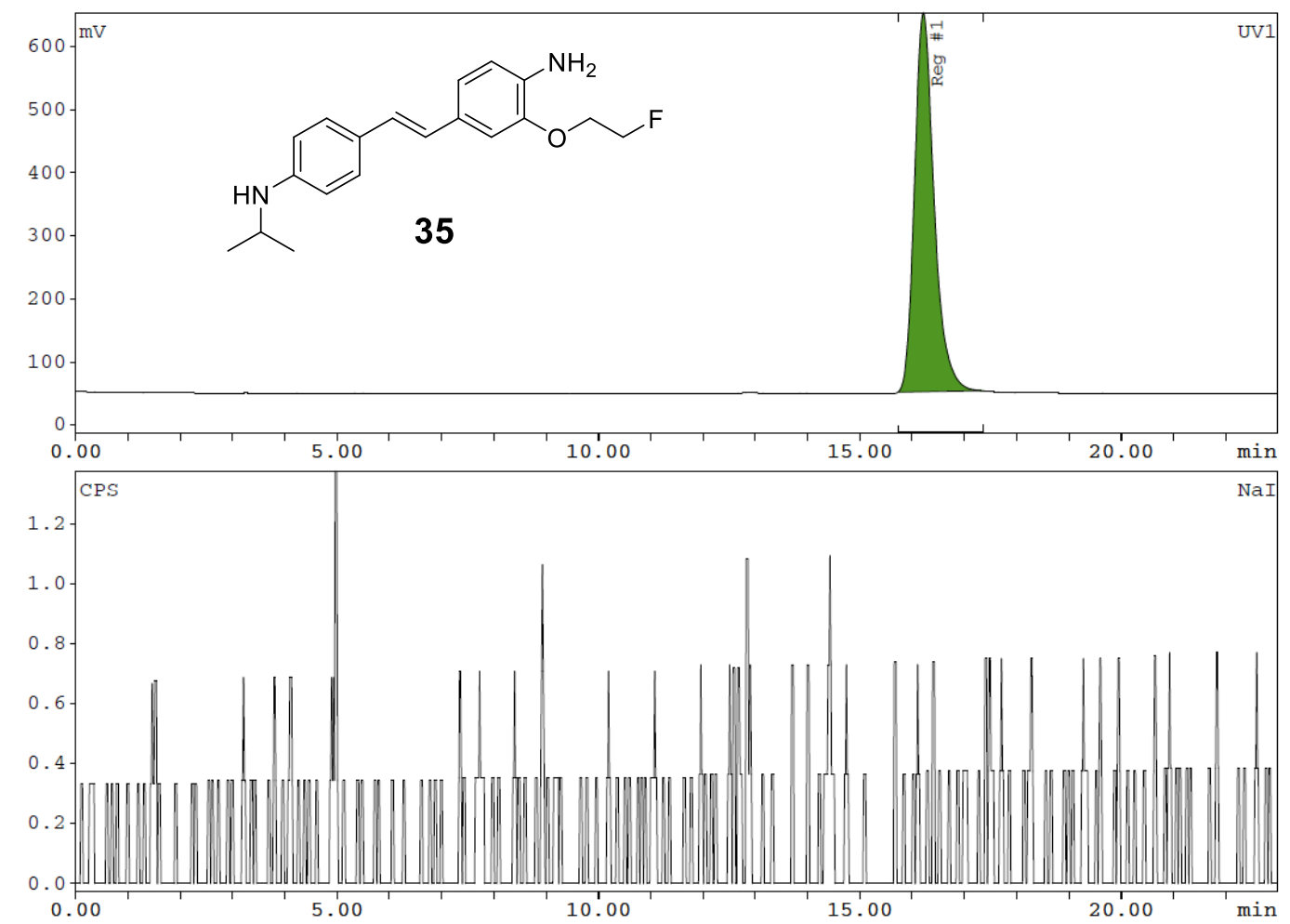

Sample description

$\begin{array}{llll}\text { Measurement: } & \text { 09092015_142C-2, injection : } & \text { 9/9/2015 } & \text { 4:11 PM } \\ \text { Method: } & \text { ANAND COMPOUND from: } & \text { 9/9/2015 } & \text { 2:51 PM }\end{array}$

Station number: $\quad 3$

Integration UV1
\begin{tabular}{|l|r|r|r|r|}
\hline Substance & $\mathbf{R} / \mathbf{T}$ & Type & Area & $\%$ Area \\
\hline & $\mathbf{m i n}$ & & $\mathbf{m V}^{*} \mathbf{s}$ & $\%$ \\
\hline Reg \#1 & 16.22 & BB & 15584.32 & 100.00 \\
Total & & & & \\
\hline
\end{tabular}


Measurement 09092015_142C-2
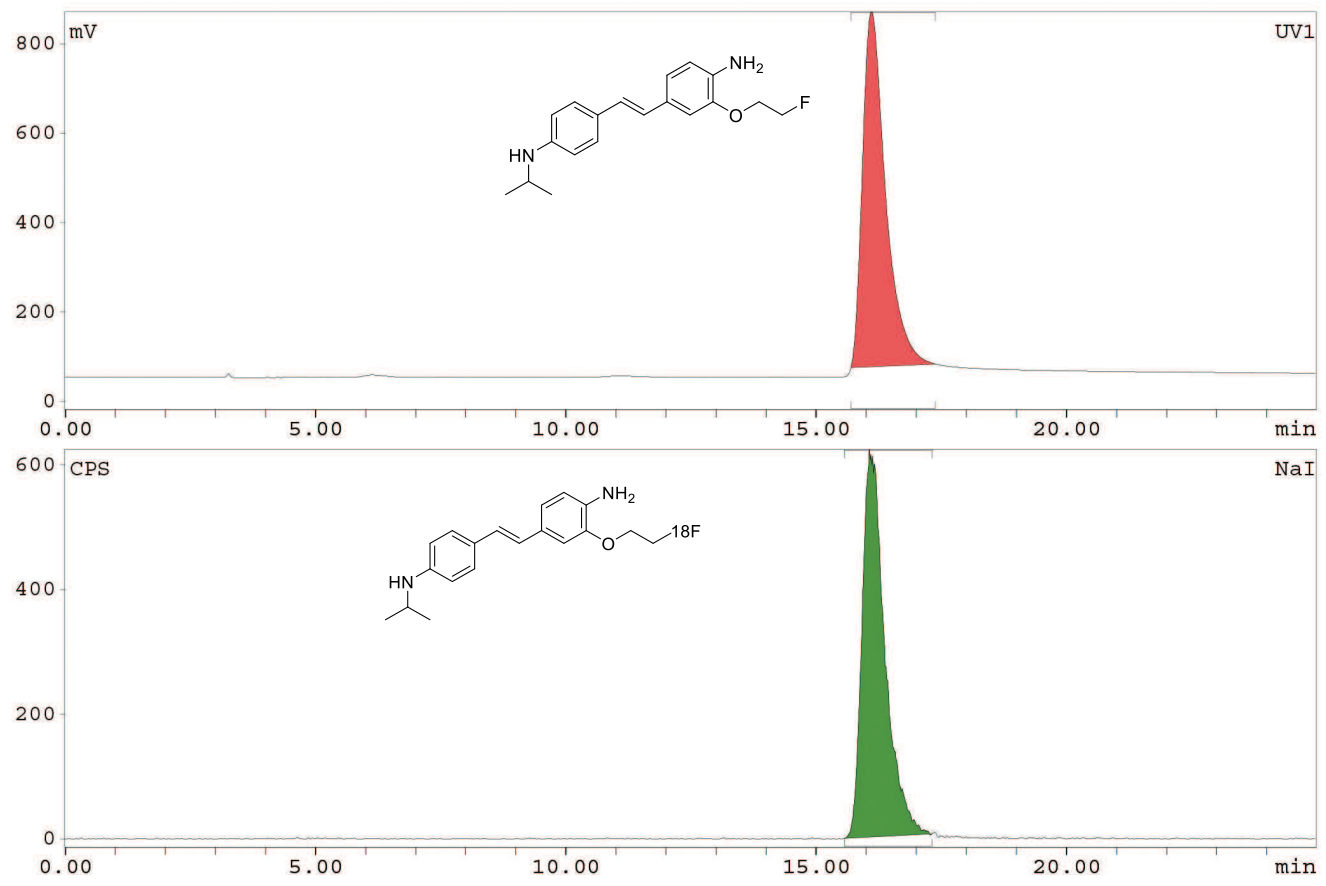

Sample description

$\begin{array}{llll}\text { Measurement: } & \text { 09092015_142C-2, injection: } & \text { 9/9/2015 4:11 PM } \\ \text { Method: } & \text { ANAND COMPOUND from: } & \text { 9/9/2015 2:51 PM } \\ \text { Station number: } & 3 & \end{array}$

Station number:

Integration $\mathrm{Nal}$

\begin{tabular}{|l|r|r|r|r|}
\hline Substance & R/T & Type & Area & \%Area \\
\hline & min & & Counts & $\%$ \\
\hline & 16.08 & BB & 19627.48 & 100.00 \\
\hline Total & & & & \\
Total area & & & 19627.48 & \\
\hline
\end{tabular}

Integration UV1

\begin{tabular}{|l|r|r|r|r|}
\hline Substance & R/T & Type & Area & \%Area \\
\hline & min & & $\mathbf{m V}^{*} \mathbf{s}$ & $\%$ \\
\hline & 16.12 & BB & 25181.90 & 100.00 \\
\hline Total & & & & \\
\hline
\end{tabular}




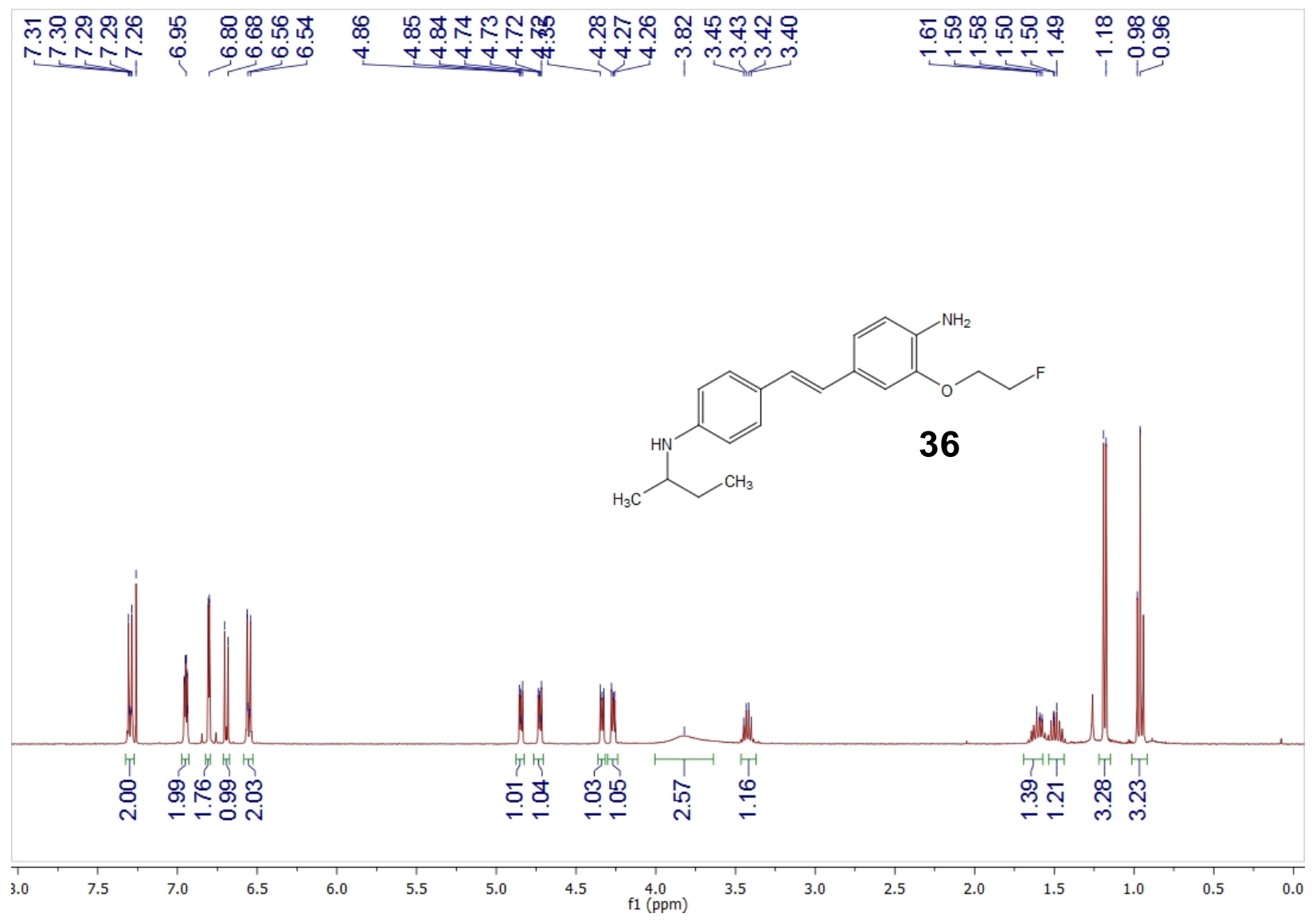




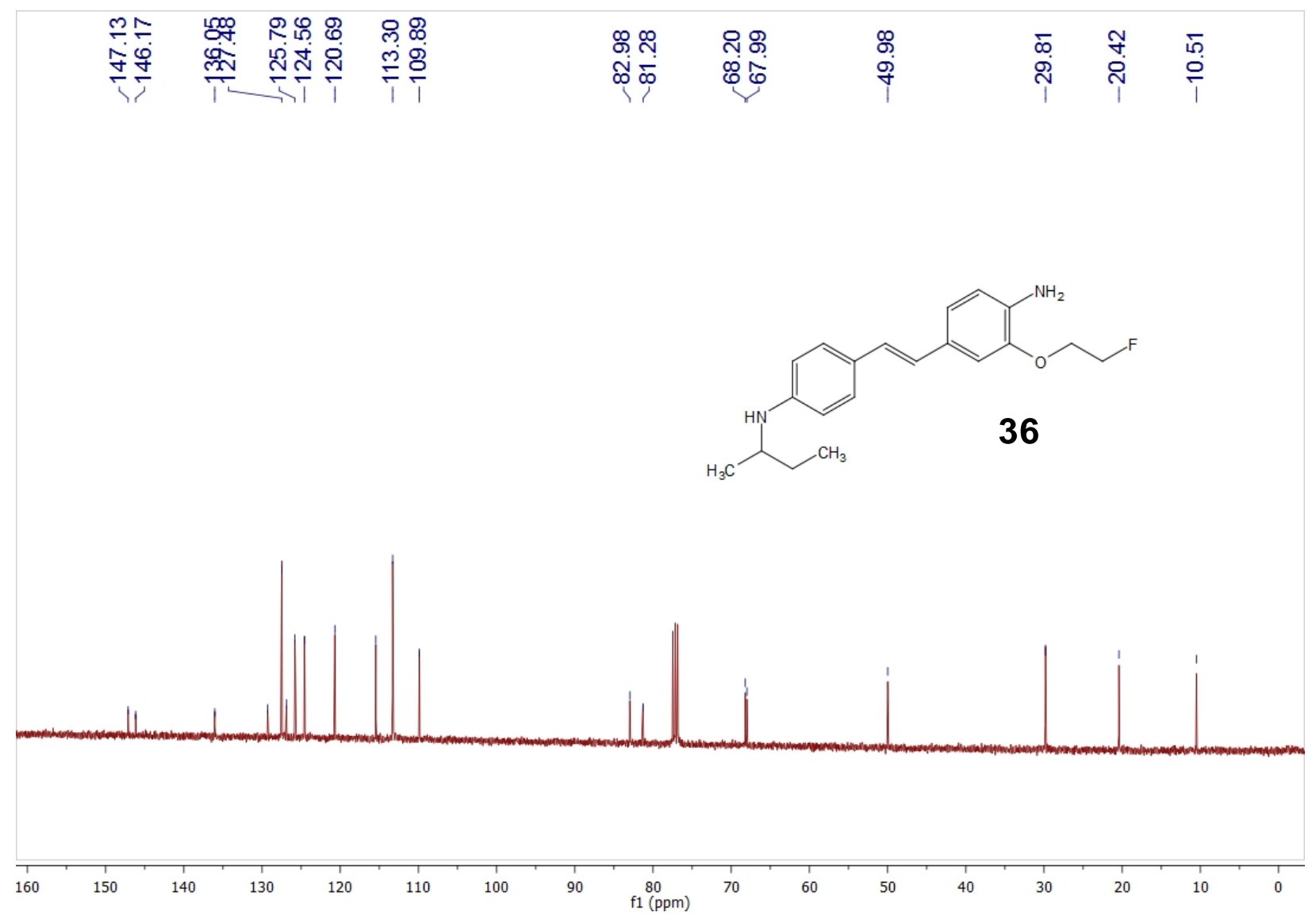




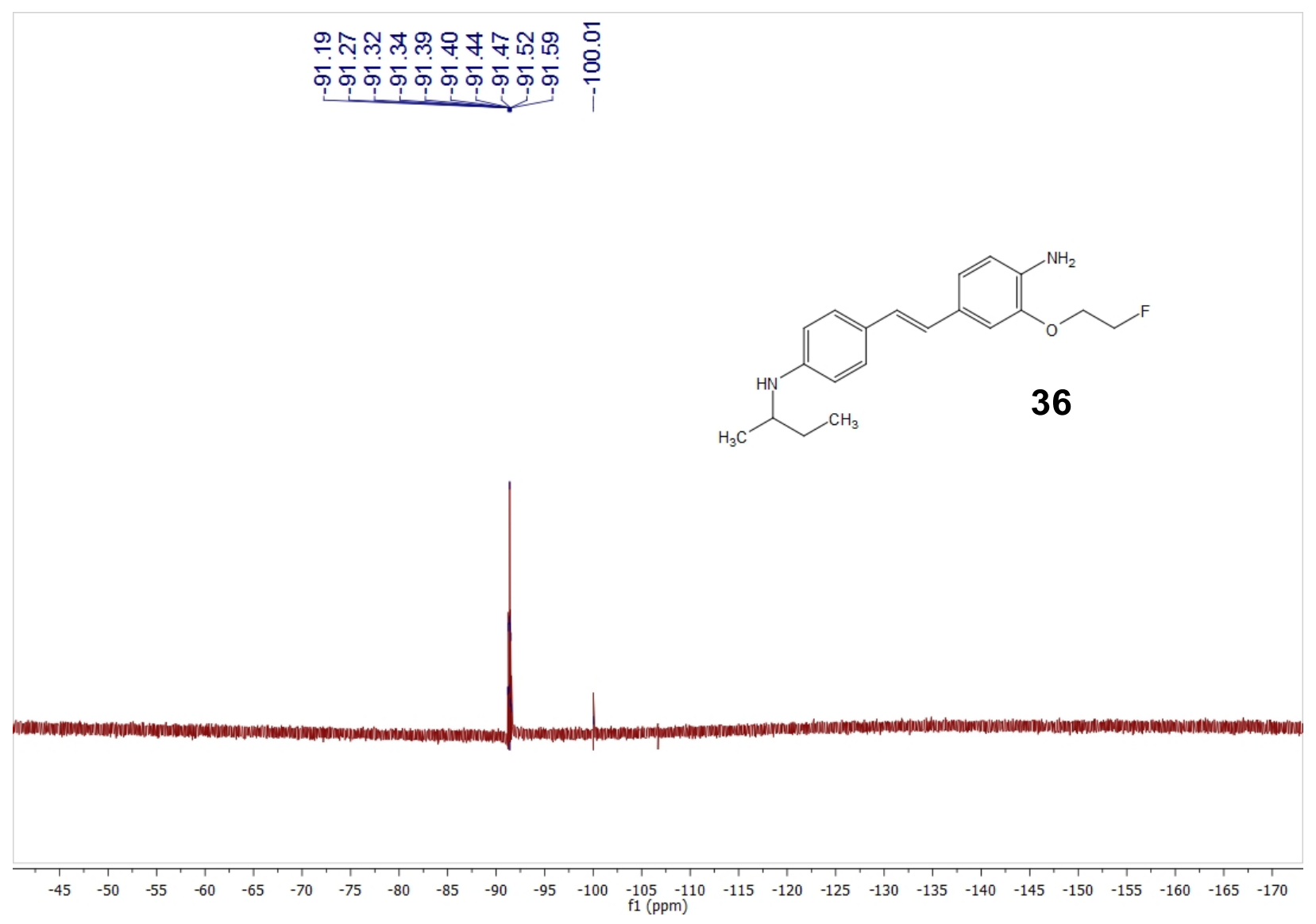




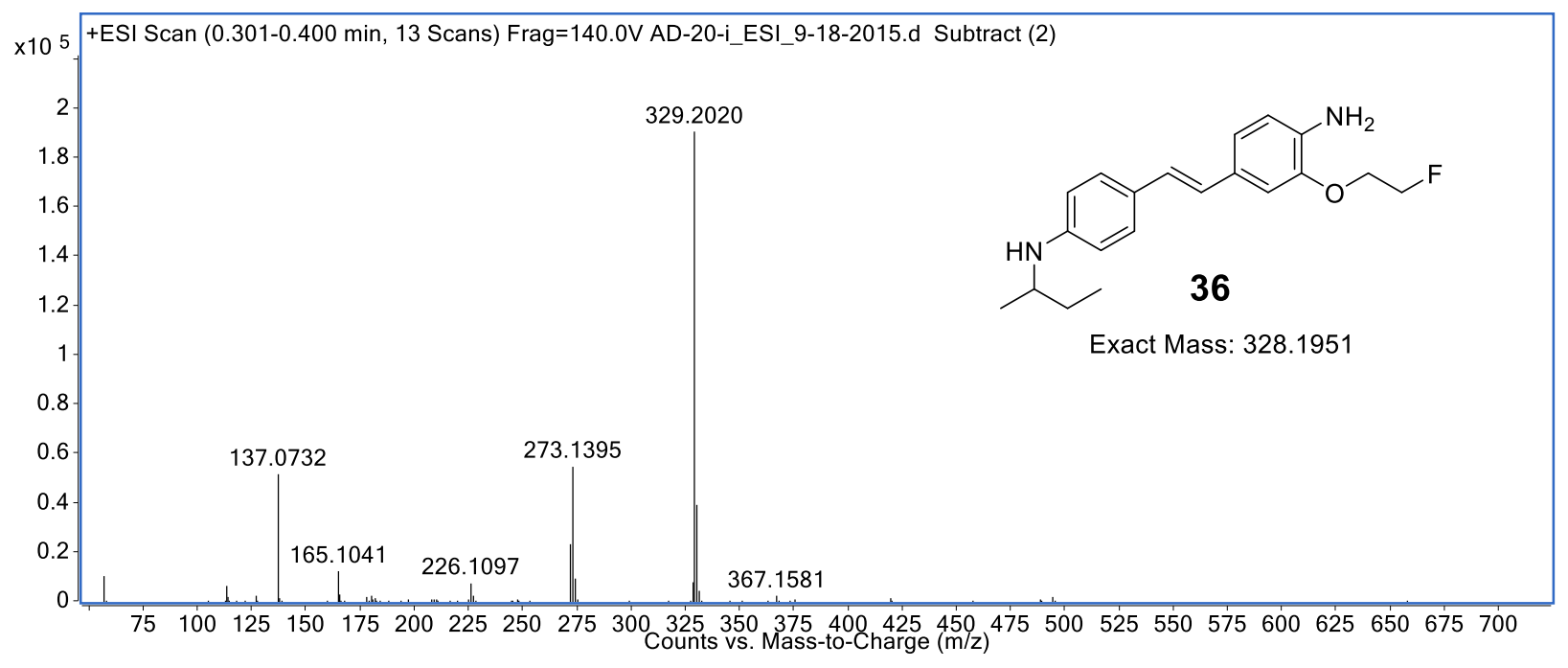



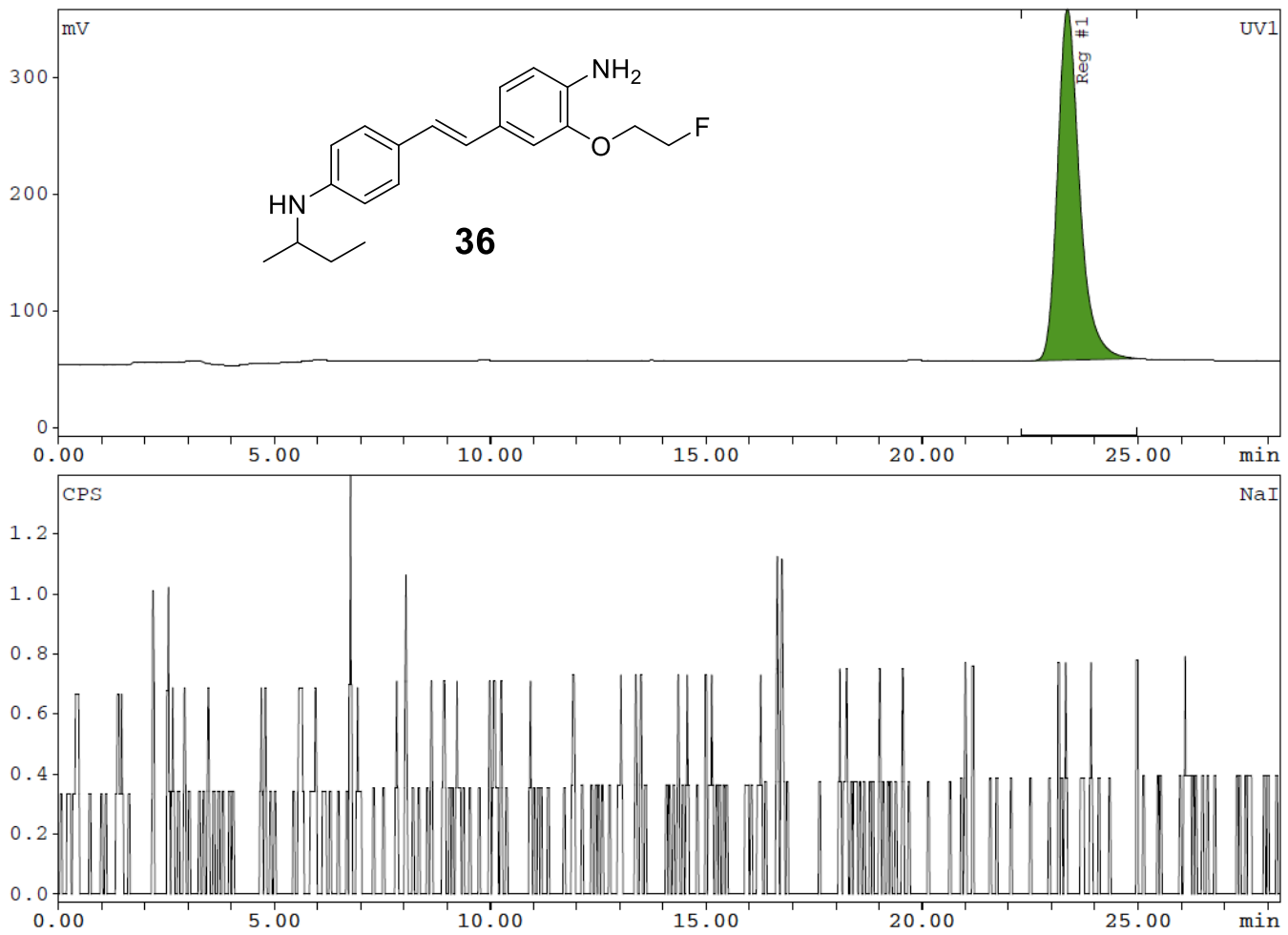

Sample description

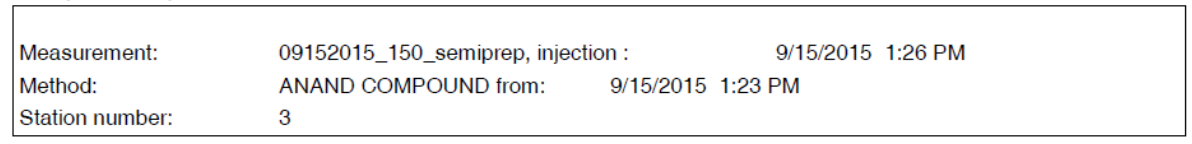

Integration UV1
\begin{tabular}{|l|r|r|r|r|}
\hline Substance & $\mathbf{R} / \mathbf{T}$ & Type & Area & $\%$ Area \\
\hline & $\mathbf{m i n}$ & & $\mathbf{m V}^{*} \mathbf{s}$ & $\%$ \\
\hline Reg \#1 & 23.37 & BB & 10552.56 & 100.00 \\
Total & & & & \\
\hline
\end{tabular}


Measurement 09242015_COI

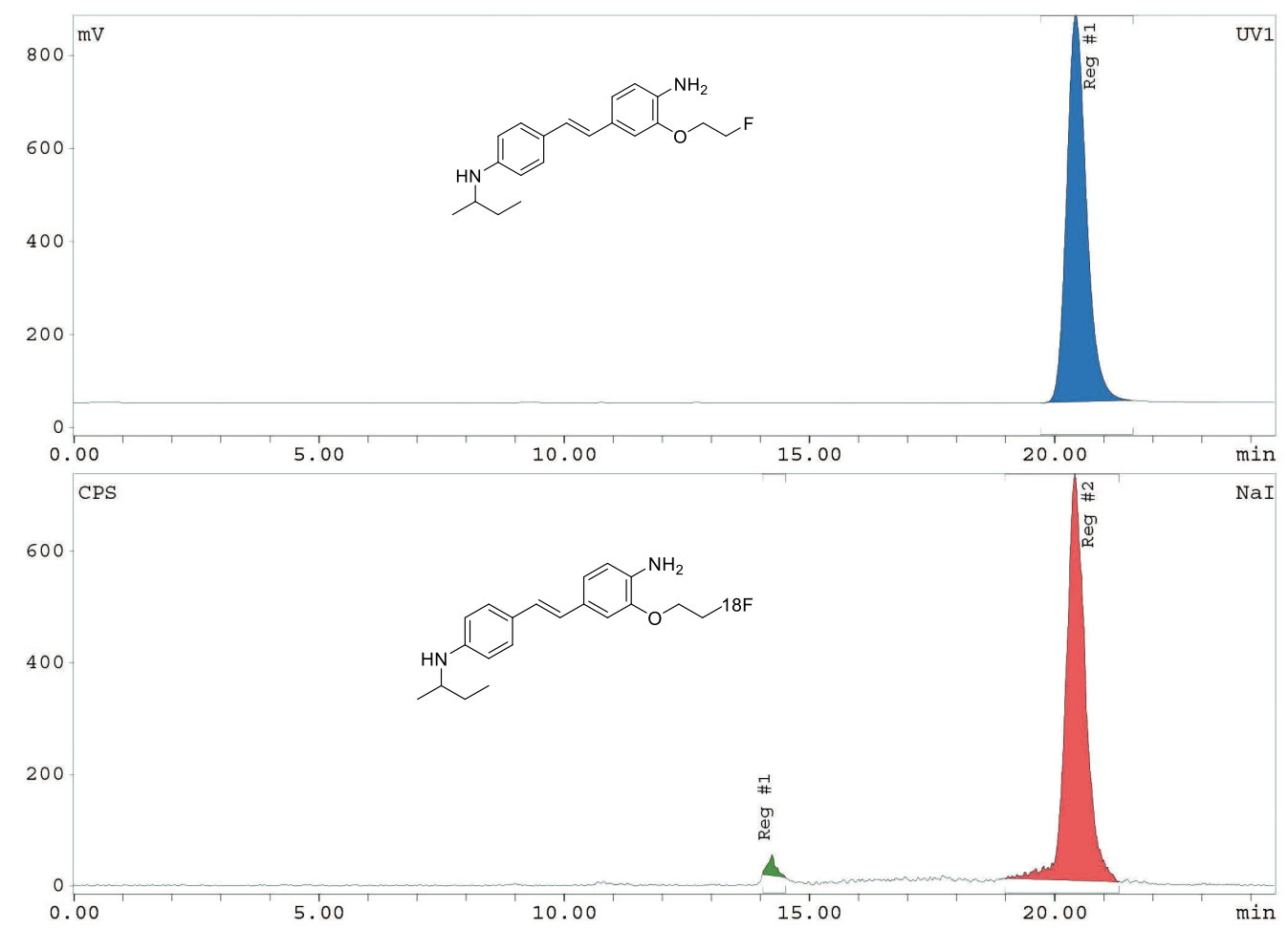

\begin{tabular}{|c|c|c|c|c|}
\hline \multicolumn{5}{|c|}{ Sample description } \\
\hline $\begin{array}{l}\text { Measurement: } \\
\text { Method: } \\
\text { Station number: }\end{array}$ & \multicolumn{3}{|c|}{$\begin{array}{l}\text { 09242015_134, injection: } \\
\text { ANAND COMPOUND from: } \\
3\end{array}$} & $\begin{array}{l}9 / \\
9 /\end{array}$ \\
\hline \multicolumn{5}{|l|}{ Integration Nal } \\
\hline \multirow[t]{2}{*}{ Substance } & $R / T$ & Type & Area & $\%$ Area \\
\hline & $\min$ & & Counts & $\%$ \\
\hline Reg \#1 & 14.23 & BB & 419.44 & 2.06 \\
\hline Reg \#2 & 20.40 & BB & 19951.66 & 97.94 \\
\hline Total & & & 20371.09 & \\
\hline Total area & & & 26712.50 & \\
\hline \multicolumn{5}{|l|}{ Integration UV1 } \\
\hline \multirow[t]{2}{*}{ Substance } & $R / T$ & Type & Area & \%Area \\
\hline & $\min$ & & $\mathbf{m V}^{*} \mathbf{s}$ & $\%$ \\
\hline Reg \#1 & 20.43 & BB & 23384.29 & 100.00 \\
\hline Total & & & 23384.29 & \\
\hline
\end{tabular}




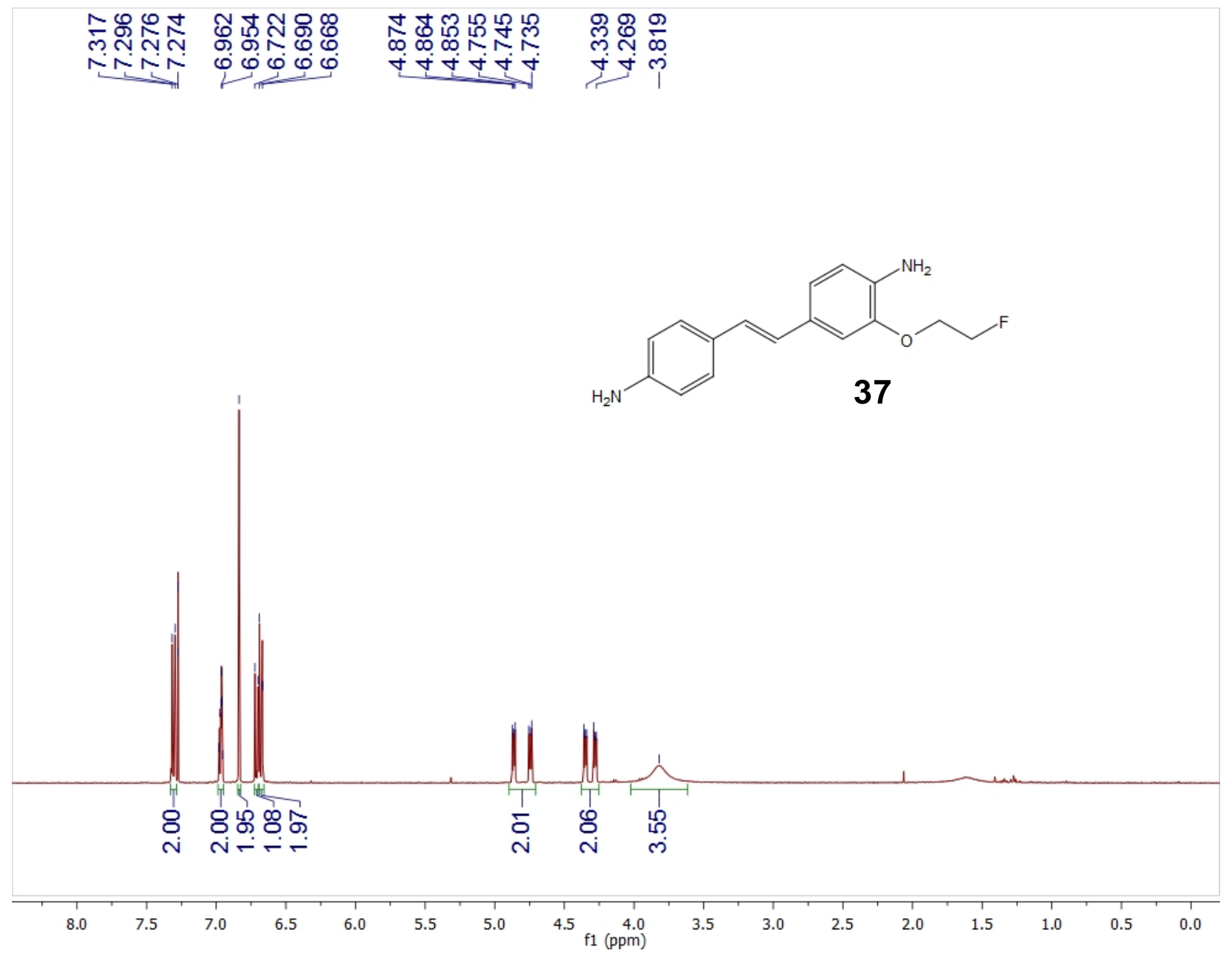




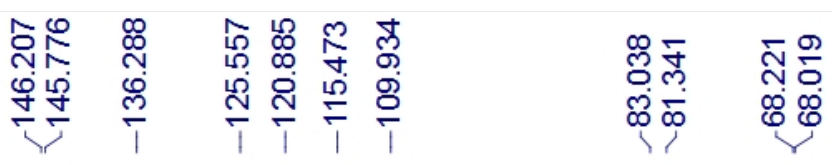<smiles>Nc1ccc(/C=C/c2ccc(N)c(OCCF)c2)cc1</smiles>
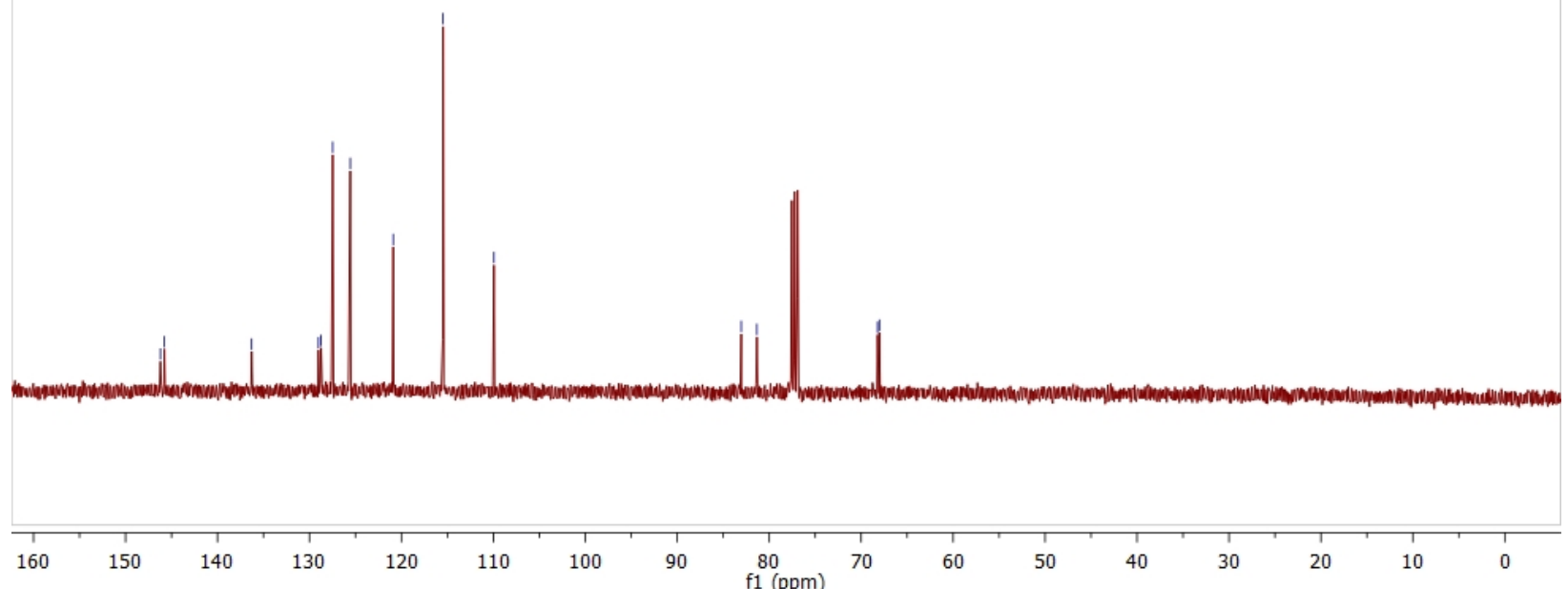


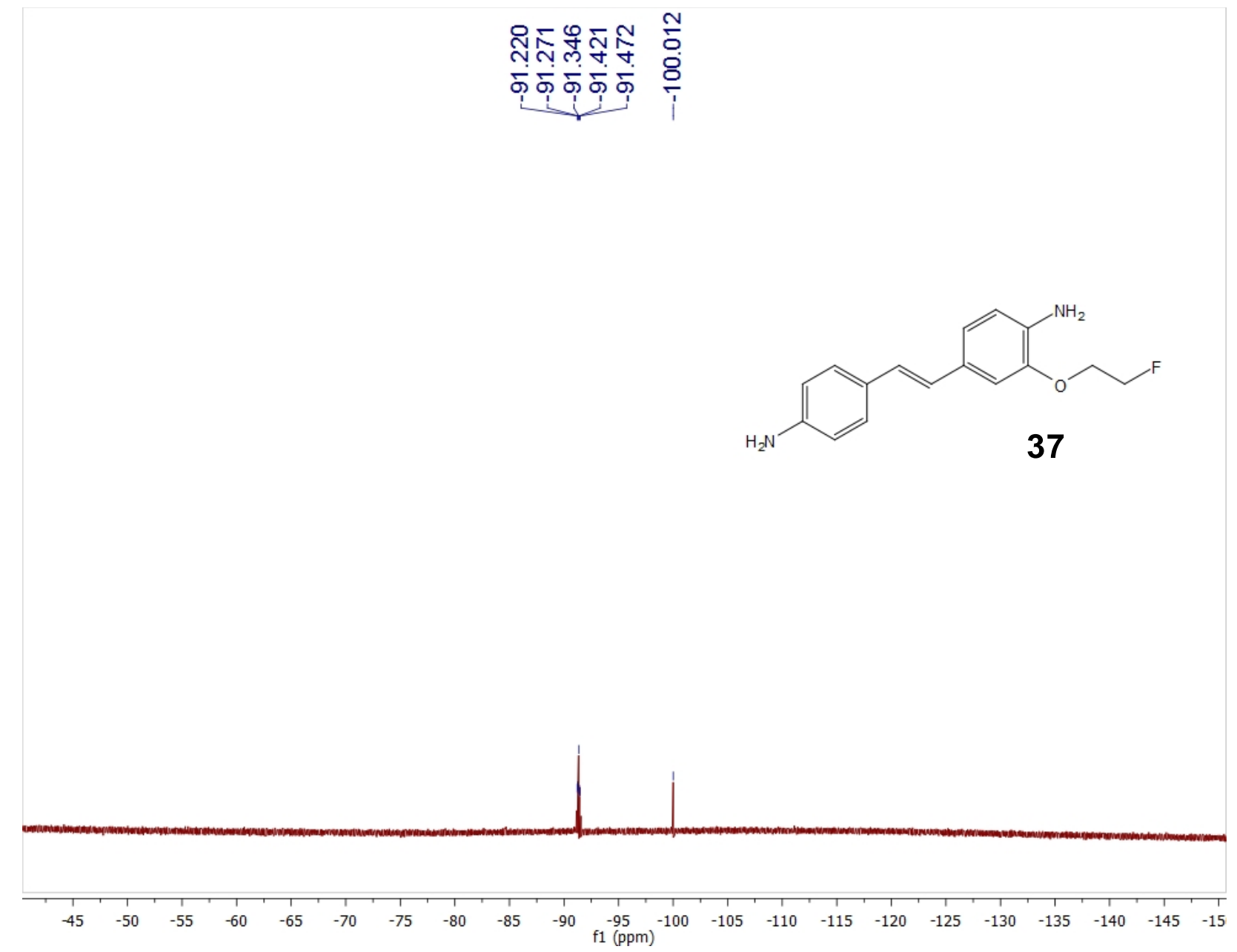




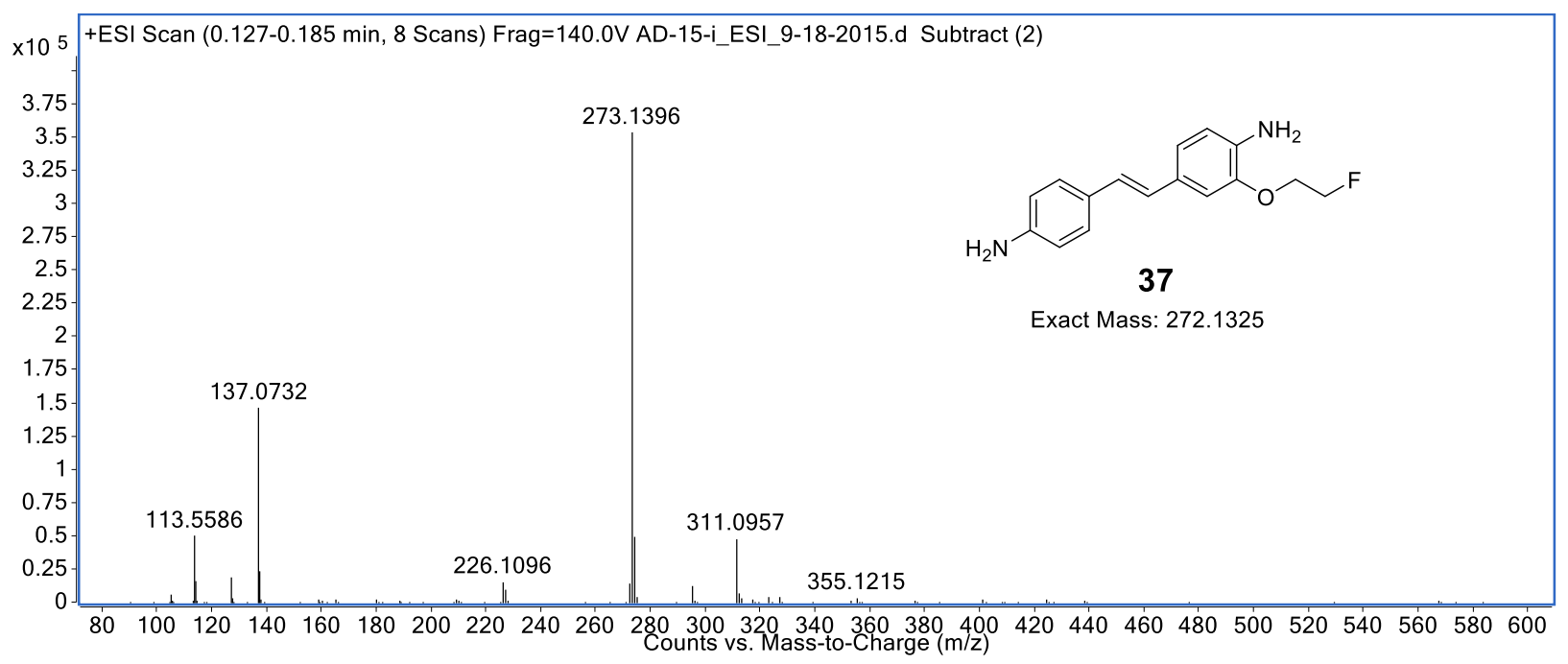


Measurement 09302015_83

PETNET

Page 1/1

C:IGINA_LCVANAND COMPOUNDI09302015_83

Print date: 9/30/2015
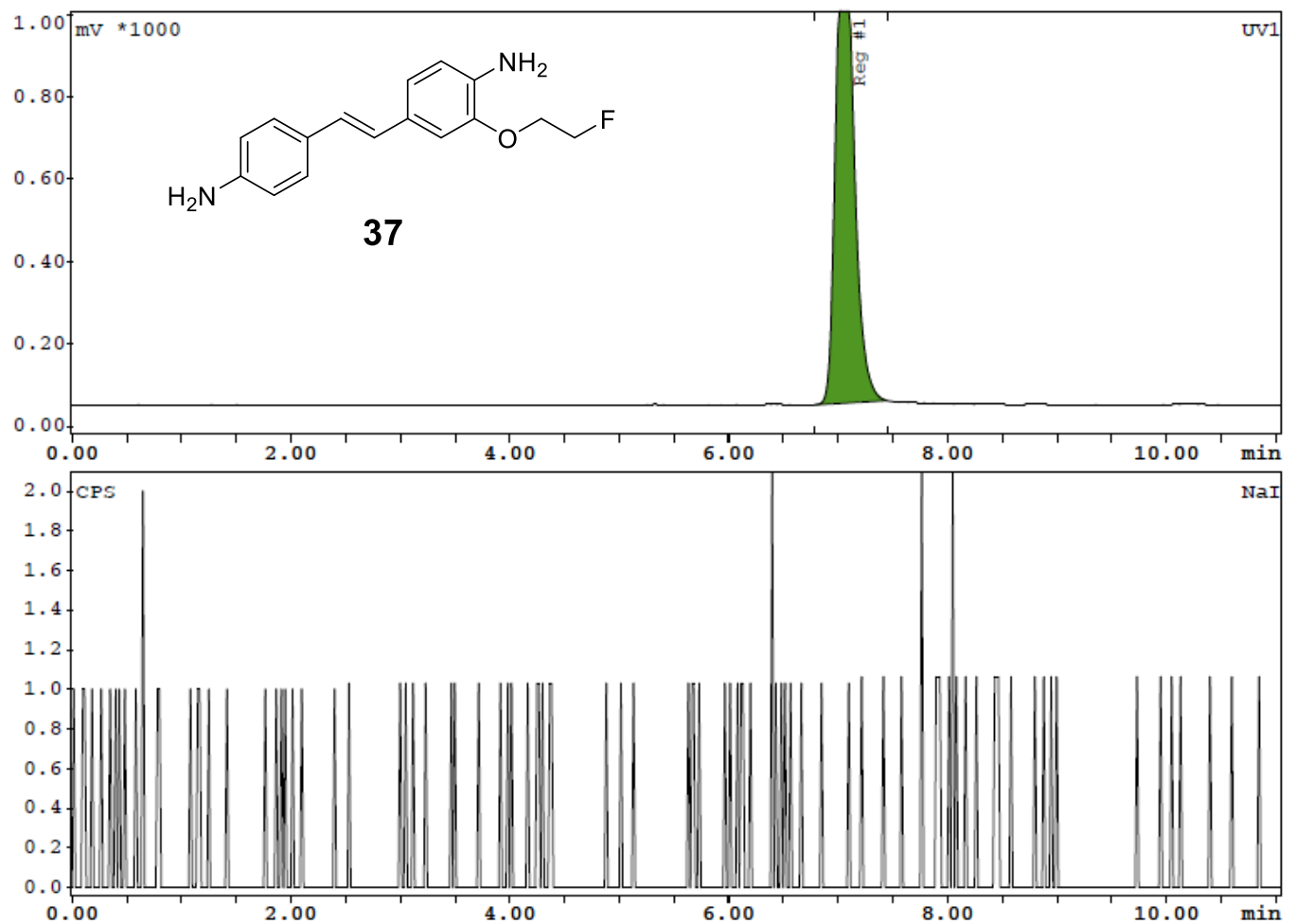

Sample description

\begin{tabular}{|c|c|c|}
\hline Measurement: & 09302015_83, injection : & 9/30/2015 4:08 PM \\
\hline Method: & ANAND COMPOUND from: & 9/30/2015 3:24 PM \\
\hline
\end{tabular}

Integration UV1

\begin{tabular}{|l|r|r|r|r|}
\hline Substance & $\mathrm{R} / \mathrm{T}$ & Type & Area & \%Area \\
\hline & $\mathrm{min}$ & & $\mathrm{mV}^{\times} \mathbf{s}$ & $\%$ \\
\hline Reg \#1 & 7.07 & BB & 12443.16 & 100.00 \\
Total & & & & \\
\hline
\end{tabular}



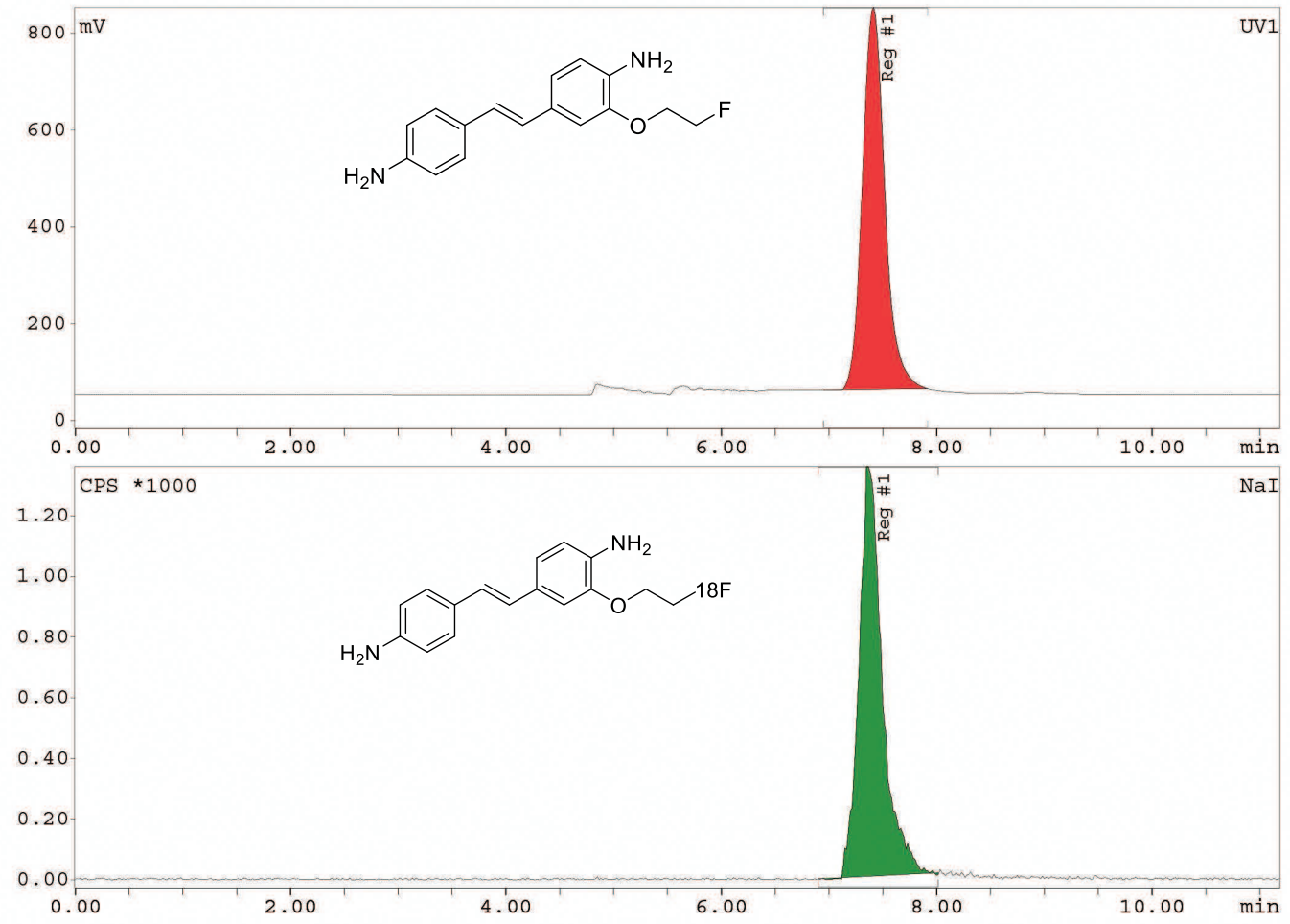

Sample description

$\begin{array}{llll}\text { Measurement: } & \text { 07162015_coin_4, injection : } & \text { 7/16/2015 3:28 PM } \\ \text { Method: } & \text { ANAND COMPOUND from: } & \text { 7/16/2015 2:31 PM } \\ \text { Station number: } & 3 & \end{array}$

Integration Nal

\begin{tabular}{|l|r|r|r|r|}
\hline Substance & R/T & Type & Area & \%Area \\
\hline Reg \#1 & min & & Counts & \% \\
\hline Total & 7.38 & BB & 20318.73 & 100.00 \\
\hline Total area & & & & \\
\hline
\end{tabular}

Integration UV1

\begin{tabular}{|l|r|r|r|r|}
\hline Substance & R/T & Type & Area & \%Area \\
\hline Reg \#1 & min & & $\mathbf{m V}^{*} \mathbf{s}$ & $\%$ \\
\hline Total & 7.42 & BB & 11347.90 & 100.00 \\
\hline & & & & \\
\hline
\end{tabular}




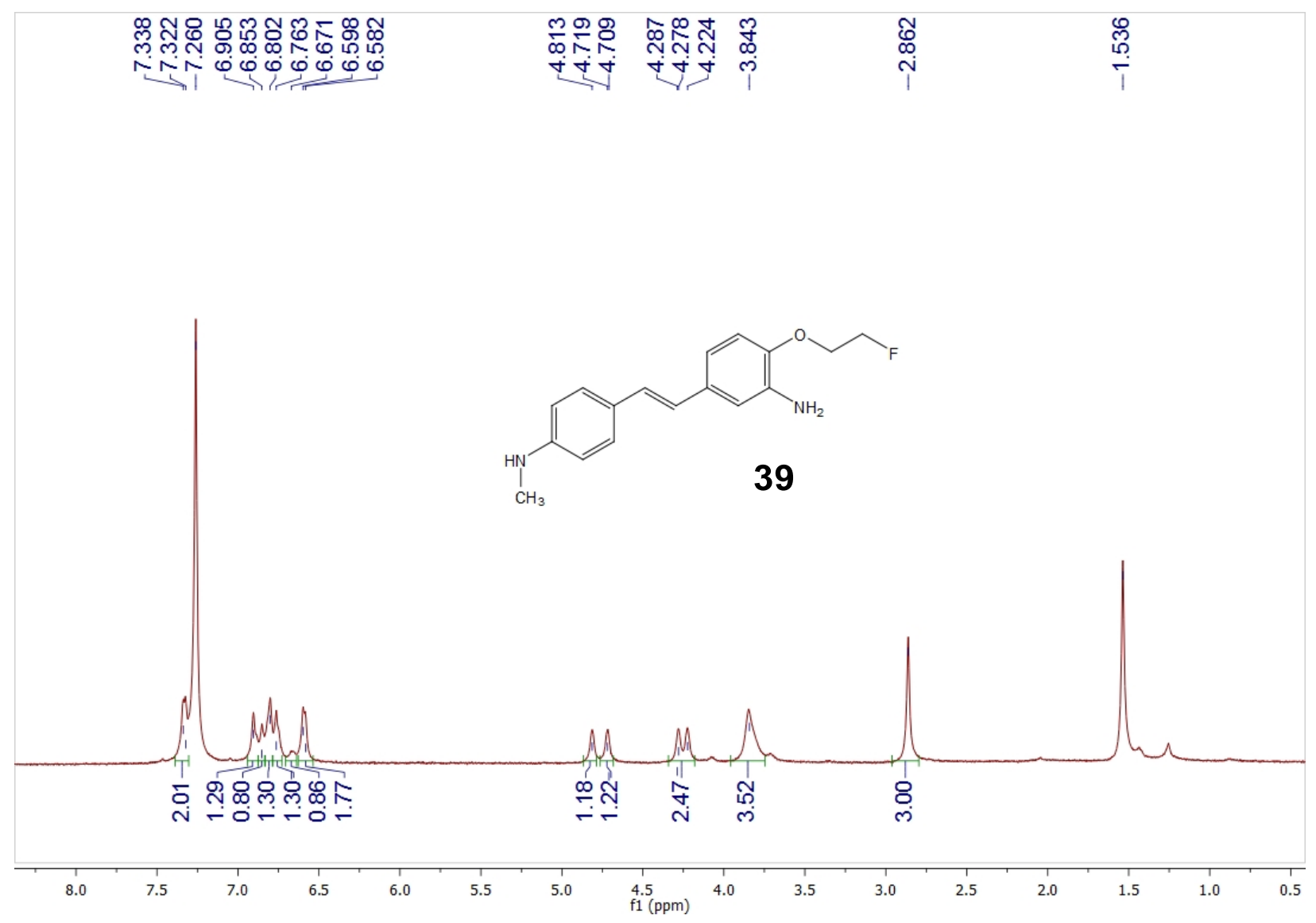



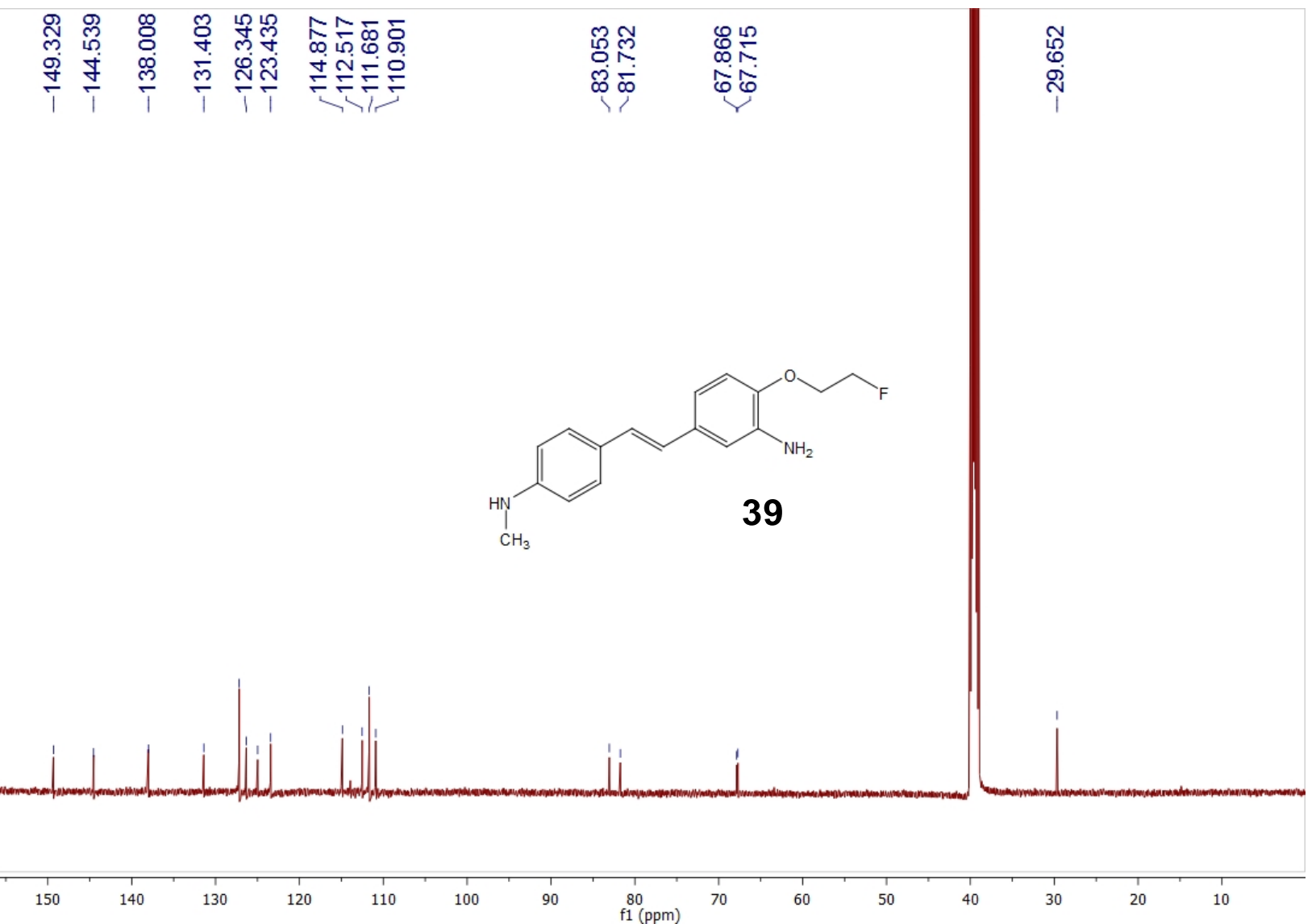


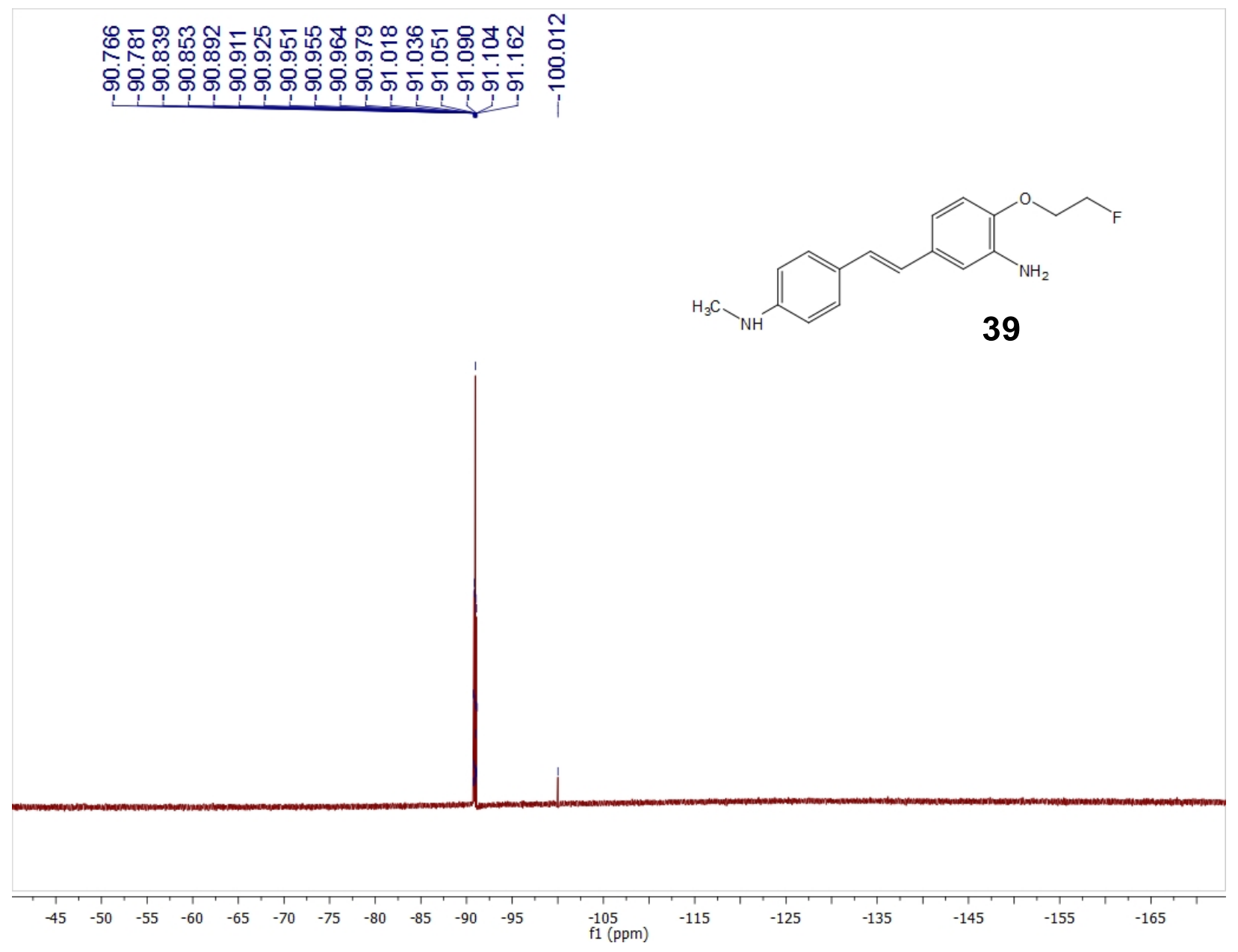




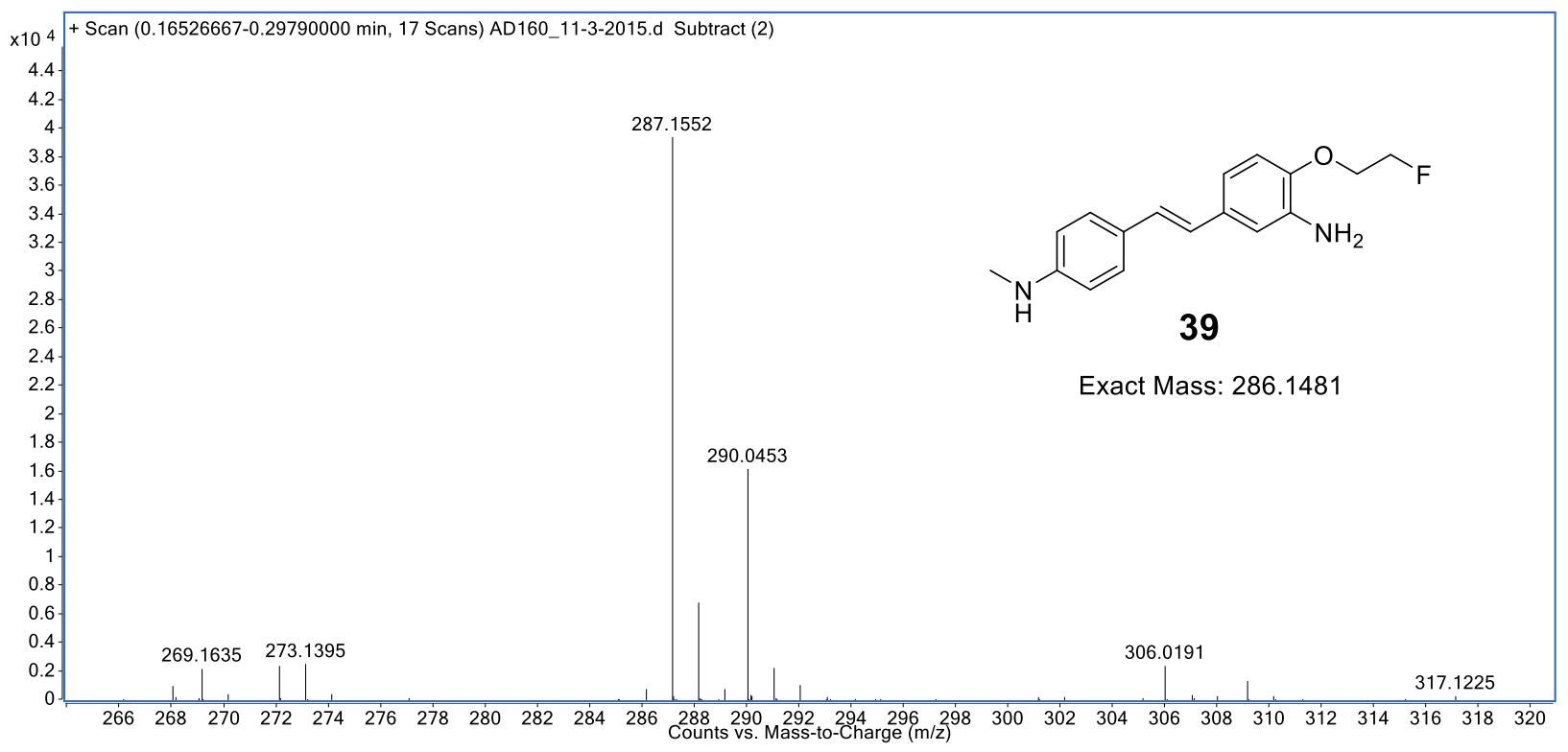




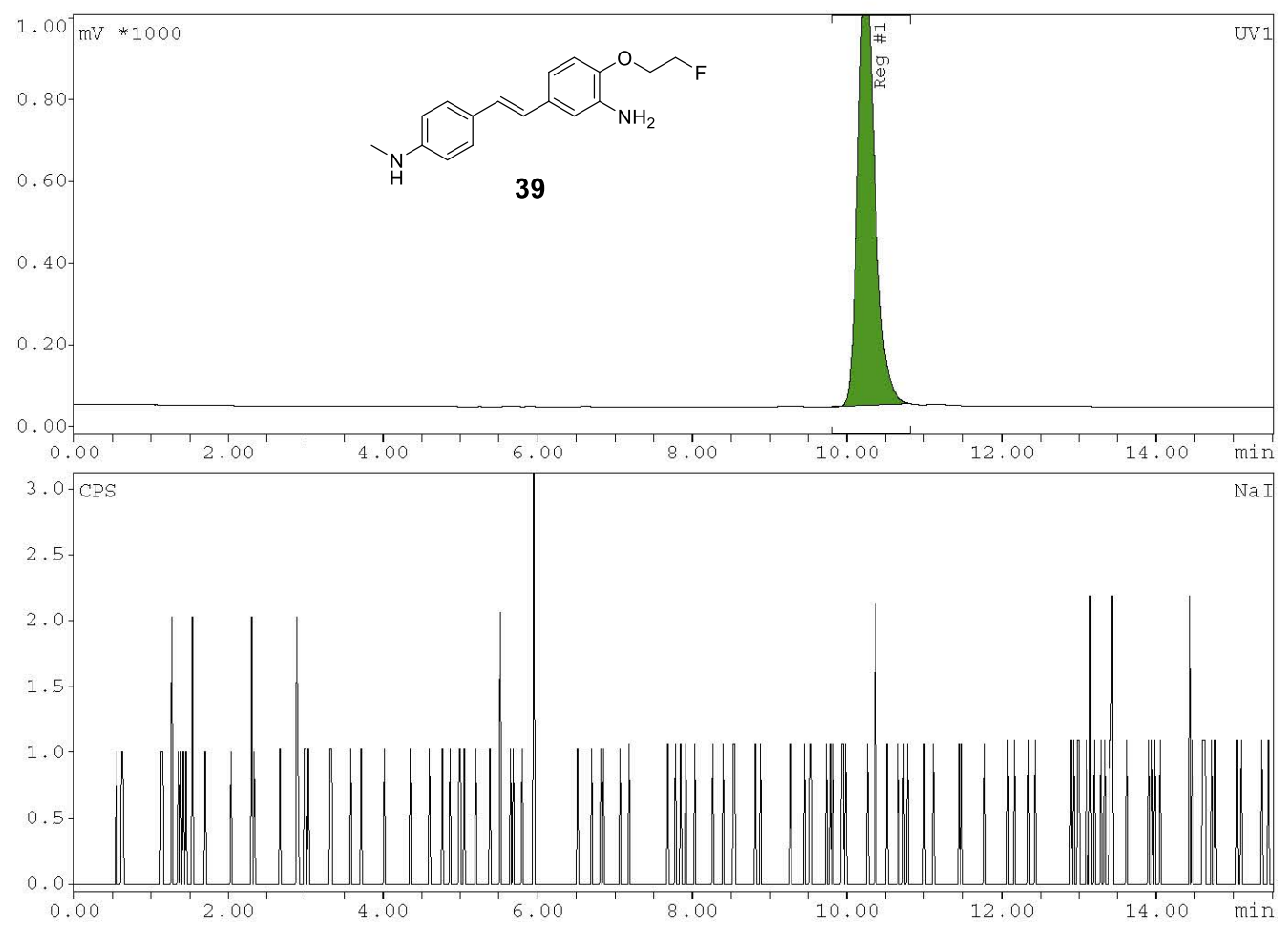

Sample description

$\begin{array}{llll}\text { Measurement: } & 01192016 \_39, \text { injection : } & \text { 1/19/2016 } & 3: 14 \text { PM } \\ \text { Method: } & \text { ANAND COMPOUND from: } & 1 / 19 / 2016 & 3: 13 \text { PM } \\ \text { Station number: } & 3 & & \end{array}$

Integration UV1

\begin{tabular}{|l|r|r|r|r|}
\hline Substance & $\mathbf{R} / \mathbf{T}$ & Type & Area & \%Area \\
\hline & $\mathbf{m i n}$ & & $\mathbf{m V}^{*} \mathbf{s}$ & $\%$ \\
\hline Reg \#1 & 10.25 & BB & 15870.76 & 100.00 \\
Total & & & & \\
\hline
\end{tabular}


Measurement 09232015_142
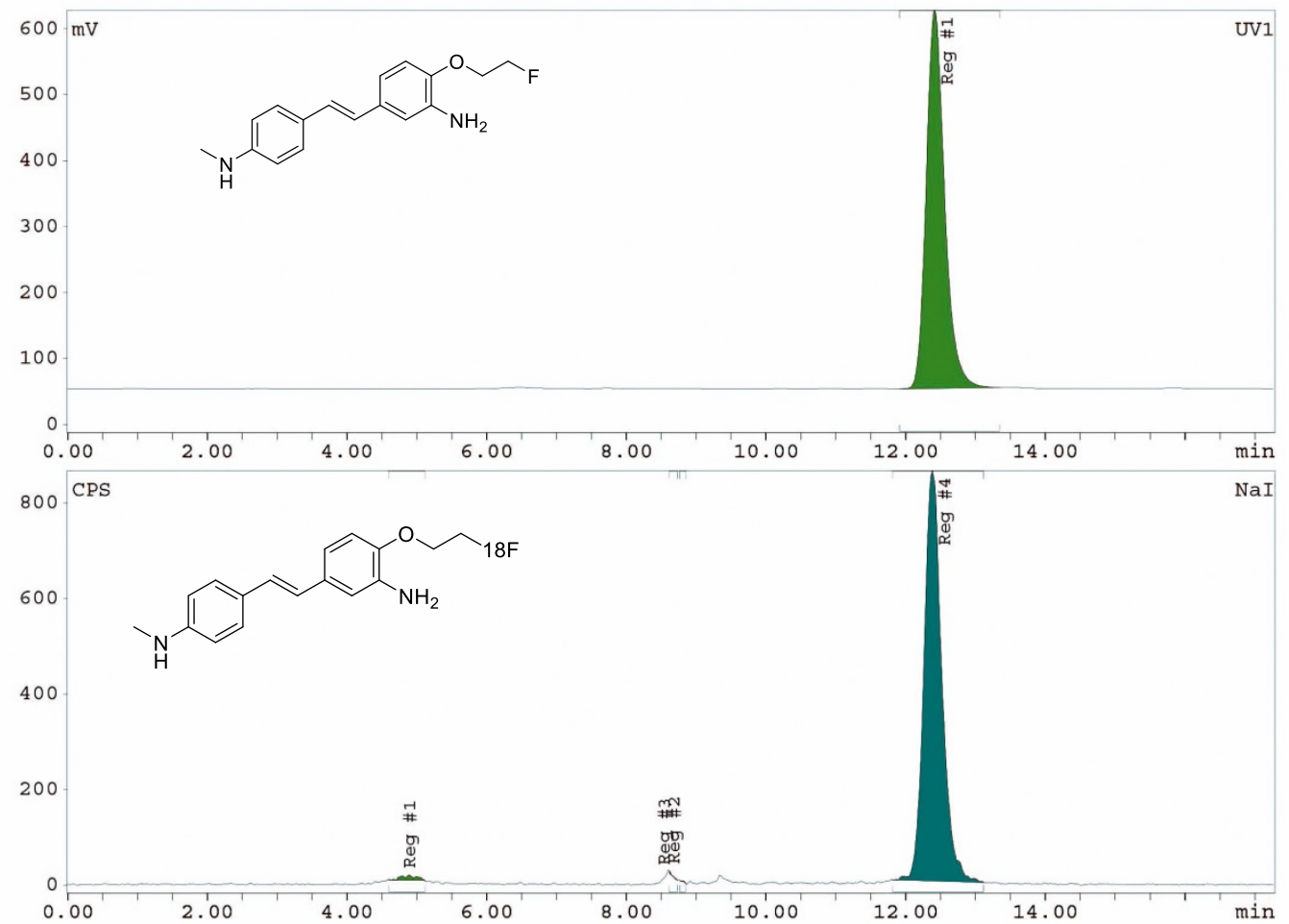

Sample description

\begin{tabular}{|c|c|c|c|c|}
\hline Measurement: & \multicolumn{4}{|c|}{ 09232015_142, injection : } \\
\hline Method: & \multicolumn{4}{|c|}{ ANAND COMPOUND from: } \\
\hline Station number: & \multicolumn{4}{|c|}{3} \\
\hline \multicolumn{5}{|l|}{ Integration Nal } \\
\hline \multirow[t]{2}{*}{ Substance } & $R / T$ & Type & Area & $\%$ Area \\
\hline & $\min$ & & Counts & $\%$ \\
\hline Reg \#1 & 4.88 & BB & 163.31 & 1.06 \\
\hline Reg \#2 & 8.67 & BB & 11.11 & 0.07 \\
\hline Reg \#3 & 8.78 & BB & 4.59 & 0.03 \\
\hline Reg \#4 & 12.40 & BB & 15266.26 & 98.84 \\
\hline Total & & & 15445.27 & \\
\hline Total area & & & 18868.53 & \\
\hline \multicolumn{5}{|l|}{ Integration UV1 } \\
\hline \multirow[t]{2}{*}{ Substance } & $R / T$ & Type & Area & $\%$ Area \\
\hline & $\min$ & & $m V^{*} s$ & $\%$ \\
\hline Reg \#1 & 12.42 & BB & 10429.92 & 100.00 \\
\hline Total & & & 10429.92 & \\
\hline
\end{tabular}


Table 1. Ex vivo staining of all compounds

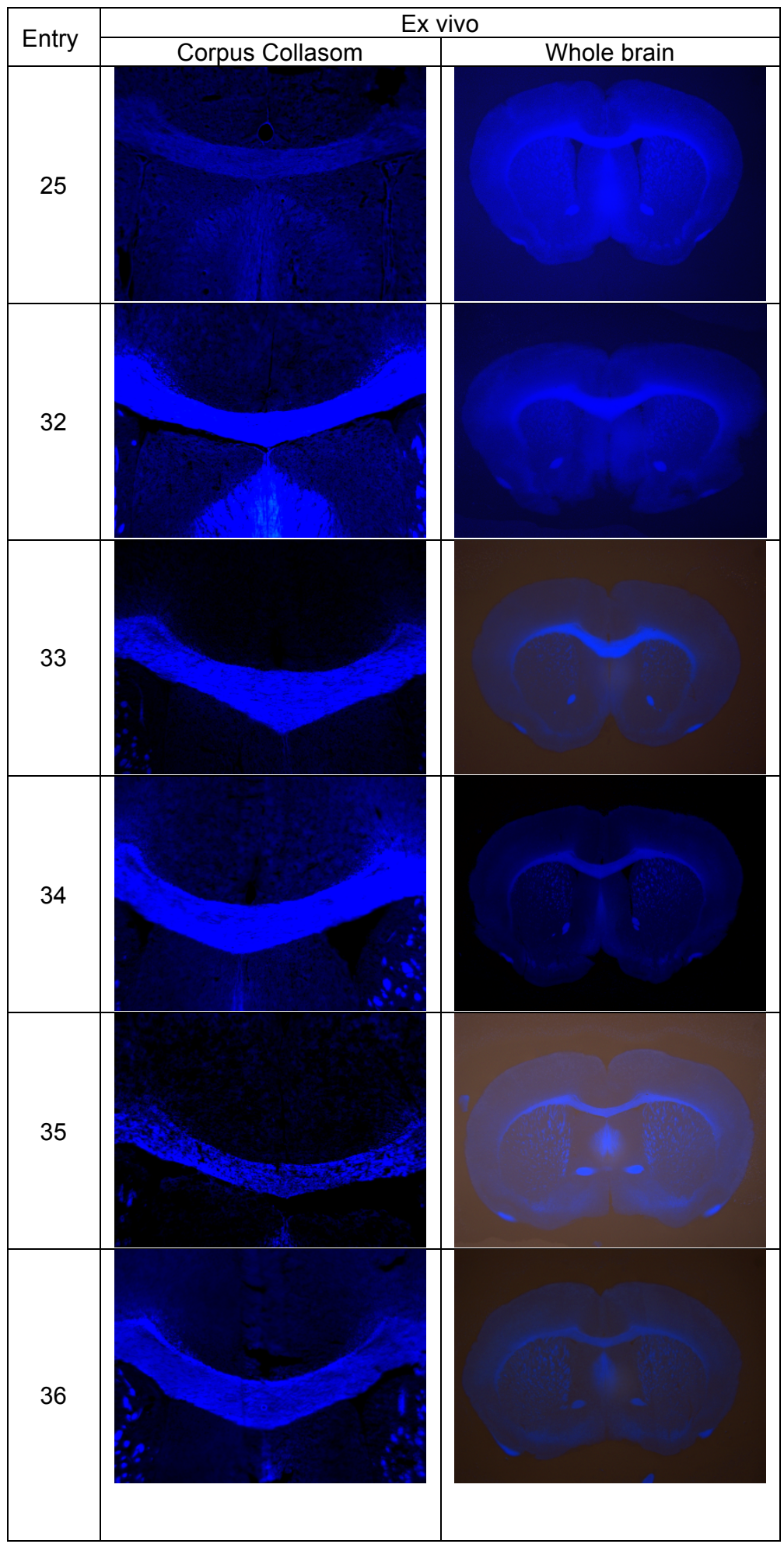




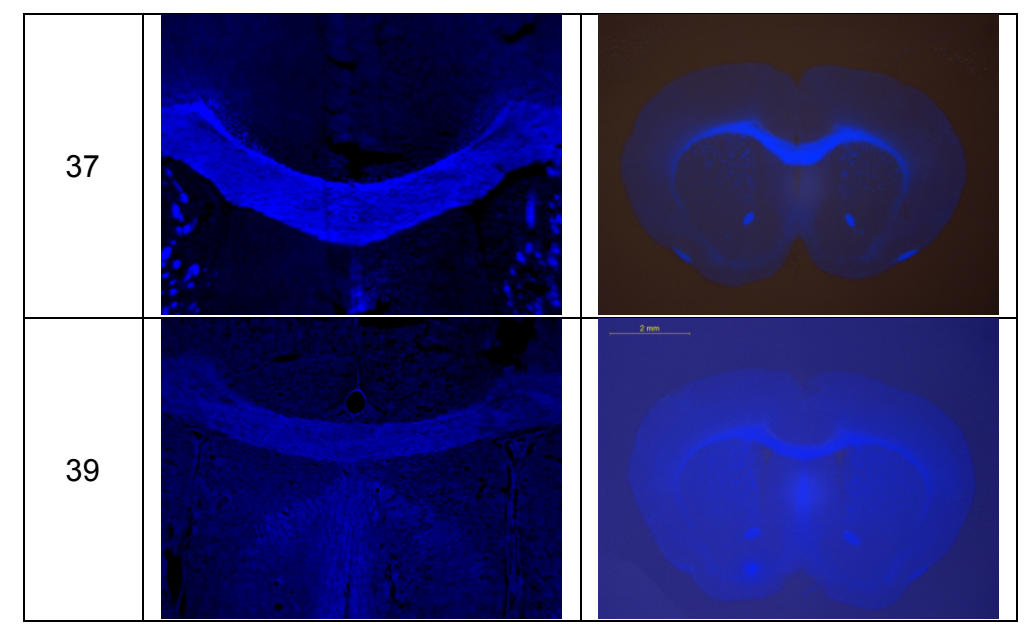


mircoPET/CT 3D movie of the whole brain and part of spinal cord imaging for the compound 32

\section{国)}

18F-32 MIP imaging.mp4 